\title{
The magnetic field in the solar atmosphere
}

\author{
Thomas Wiegelmann • Julia K. Thalmann • \\ Sami K. Solanki
}

Received: 16 October 2014 / Published online: 16 November 2014

C The Author(s) 2014. This article is published with open access at Springerlink.com

\begin{abstract}
This publication provides an overview of magnetic fields in the solar atmosphere with the focus lying on the corona. The solar magnetic field couples the solar interior with the visible surface of the Sun and with its atmosphere. It is also responsible for all solar activity in its numerous manifestations. Thus, dynamic phenomena such as coronal mass ejections and flares are magnetically driven. In addition, the field also plays a crucial role in heating the solar chromosphere and corona as well as in accelerating the solar wind. Our main emphasis is the magnetic field in the upper solar atmosphere so that photospheric and chromospheric magnetic structures are mainly discussed where relevant for higher solar layers. Also, the discussion of the solar atmosphere and activity is limited to those topics of direct relevance to the magnetic field. After giving a brief overview about the solar magnetic field in general and its global structure, we discuss in more detail the magnetic field in active regions, the quiet Sun and coronal holes.
\end{abstract}

Keywords Sun · Photosphere - Chromosphere - Corona · Magnetic field · Active region · Quiet Sun · Coronal holes

T. Wiegelmann $(\bowtie) \cdot$ S. K. Solanki

Max-Planck-Institut für Sonnensystemforschung, Justus-von-Liebig-Weg 3, 37077 Göttingen, Germany

e-mail: wiegelmann@mps.mpg.de

J. K. Thalmann

Institute for Physics/IGAM, University of Graz, Universitätsplatz 5/II, 8010 Graz, Austria e-mail: julia.thalmann@uni-graz.at

S. K. Solanki

School of Space Research, Kyung Hee University, Yongin, Gyeonggi 446-701, Republic of Korea e-mail: solanki@mps.mpg.de 


\section{Contents}

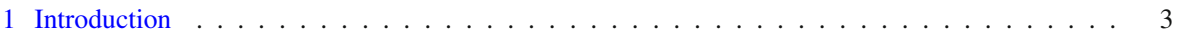

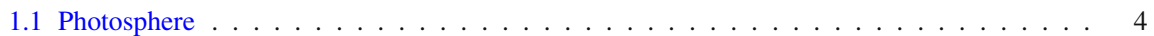

1.1.1 Magnetic flux emergence . . . . . . . . . . . . . . . . . . 5

1.1.2 Spatial properties of magnetic features . . . . . . . . . . . . . . . 6

1.1 .3 Origin of internetwork fields . . . . . . . . . . . . . . . 6

1.1.4 Temporal evolution of the magnetic field . . . . . . . . . . . . . . . . . . 7

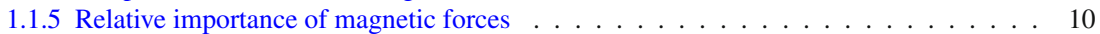

1.2 Chromosphere . . . . . . . . . . . . . . . . . . . . . . . 11

1.2.1 Characteristic chromospheric magnetic structures . . . . . . . . . . . . . . 11

1.2.2 Indirect tracing of chromospheric fields . . . . . . . . . . . . . . . . . . . 13

1.2.3 Plasma- $\beta$ in the chromosphere . . . . . . . . . . . . . . . . 15

1.3 Magnetic coupling from the lower solar atmosphere to the corona . . . . . . . . . . . 16

1.3.1 Magnetic canopy . . . . . . . . . . . . . . . . . . . . . . . . . . . . . . . . . .

1.4 Corona . . . . . . . . . . . . . . . . . . . . . . 18

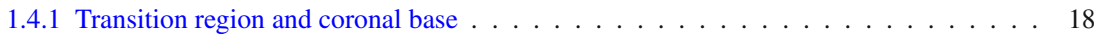

1.4.2 Morphology of coronal magnetic fields . . . . . . . . . . . . . . . . . . . . 19

2 Magnetic field modeling . . . . . . . . . . . . . . . . . . . . . . . . . . . . 19

2.1 Direct coronal magnetic field measurements . . . . . . . . . . . . . . . . . . . . . 20

2.1.1 Chromospheric magnetic field measurements in the infrared . . . . . . . . . . . . 20

2.1.2 Coronal magnetic field measurements in infrared . . . . . . . . . . . . . . . . . 21

2.1.3 Coronal magnetic field measurements at radio wavelengths . . . . . . . . . . . . . . . 21

2.2 Force-free modeling from photospheric measurements . . . . . . . . . . . . . . . . . . 22

2.2.1 Potential and linear force-free fields . . . . . . . . . . . . . . . . . . . . . . . . . . . . . . . . . . .

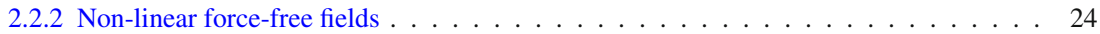

2.3 MHD models . . . . . . . . . . . . . . . . . . . . . . . . . . . . 25

2.3.1 MHD models of the coronal magnetic field . . . . . . . . . . . . . . . . . . . 25

2.3 .2 MHS models . . . . . . . . . . . . . . . . . . . . . . 26

2.3 .3 Flux transport models . . . . . . . . . . . . . . . . . . . . . . 27

2.4 Coronal stereoscopy, tomography and seismology . . . . . . . . . . . . . . . . . . . 27

2.4.1 Stereoscopy and magnetic stereoscopy . . . . . . . . . . . . . . . . . . . . . . . . . . . . . . . . . .

2.4 .2 Tomography and vector tomography . . . . . . . . . . . . . . . . . . . 28

2.4 .3 Coronal seismology . . . . . . . . . . . . . . . . . . . . . . . . . 29

3 Global coronal magnetic fields . . . . . . . . . . . . . . . . . . . . . . . . . 30

3.1 Magnetic field topology . . . . . . . . . . . . . . . . . . . . . . . . . . . . . . . . . 30

3.1 .1 Performance of PFSS models . . . . . . . . . . . . . . . . . . 30

3.1 .2 Achievements of global MHD models . . . . . . . . . . . . . . . . . . . 32

3.2 Cross-equatorial fields . . . . . . . . . . . . . . . . . . . . . . . 32

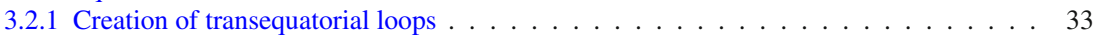

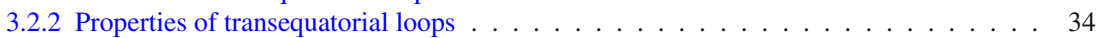

3.3 Spatio-temporal aspects of activity . . . . . . . . . . . . . . . . . . . 35

3.3.1 Cyclic changes of the coronal magnetic field . . . . . . . . . . . . . . 35

3.3 .2 Association to dynamic events . . . . . . . . . . . . . . . 36

3.4 Magnetic helicity budget . . . . . . . . . . . . . . . . . . . . . 37

3.4.1 Helicity dissipation and helicity transport . . . . . . . . . . . . . . . . . . . . . . . . . . . . . 37

3.4 .2 Hemispheric trends . . . . . . . . . . . . . . . . . . . . . . . . . . . . . . . . . . . .

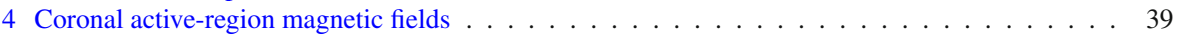

4.1 Coronal loops . . . . . . . . . . . . . . . . . . . . . . . . . . . . . 39

4.2 Local field topology . . . . . . . . . . . . . . . . . . . . . . . . . . . . . . . . . . . . . . . . . . . . . .

4.2 .1 Magnetic skeleton . . . . . . . . . . . . . . . . . . . . . . 42

4.2.2 Association to current-sheets and magnetic reconnection . . . . . . . . . . . . . . . 43

4.2 .3 Relation to dynamic phenomena . . . . . . . . . . . . . . . . . . . . . 44 44

4.3 Temporal evolution of active-region magnetic fields . . . . . . . . . . . . . . . . . . 46

4.3.1 Dynamic evolution: eruptive phenomena . . . . . . . . . . . . . . . . 46

4.4 Favorable conditions for eruptions . . . . . . . . . . . . . . . . . . . . . 47

4.4.1 Magnetic flux emergence and complex magnetic field structure . . . . . . . . . . . 47 
4.4 .2 Magnetic shear and twist . . . . . . . . . . . . . . . . . . . . . . . . . . . . . . . . . . . . . . . . . . . 48

4.5 Magnetic energy budget . . . . . . . . . . . . . . . . . . . . . . . . . . . 49

4.5 .1 Energy buildup and storage . . . . . . . . . . . . . . . . . . . . . 49

4.5 .2 Relation to flare productivity . . . . . . . . . . . . . . . . . 50

4.5 .3 Energy release . . . . . . . . . . . . . . . . . . . . 51

4.5.4 Coronal implosion and photospheric response . . . . . . . . . . . . . . . 52

4.6 Magnetic helicity budget . . . . . . . . . . . . . . . . . . . . . . . . . 54

4.6 .1 Helicity buildup and storage . . . . . . . . . . . . . . . . . . . . 55

4.6 .2 Relation to flare productivity . . . . . . . . . . . . . . . . . . . . . . . . . . 56

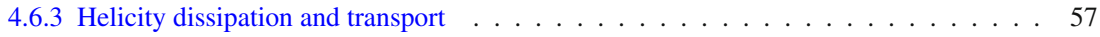

5 Quiet-Sun magnetic fields . . . . . . . . . . . . . . . . . . . . . . . . 58

$5.13 \mathrm{D}$ magnetic field structure in the quiet Sun . . . . . . . . . . . . . . . . . 59

5.1 .1 Photospheric quiet-Sun loops . . . . . . . . . . . . . . . . . . . . 60

5.2 Associated photospheric fields and the response on the upper atmosphere . . . . . . . . . . 60

5.2 .1 Doubts about the concept of a magnetic canopy . . . . . . . . . . . . . . . . 61

5.2 .2 Network and internetwork magnetic loops . . . . . . . . . . . . . . . . . . . 62

5.2 .3 Magnetic carpet . . . . . . . . . . . . . . . . . . . . . 63

5.2.4 Role of magnetic fields in coronal and chromospheric heating . . . . . . . . . . . . 64

5.2 .5 Force-freeness of quiet-Sun magnetic fields . . . . . . . . . . . . . . . . . . . . 6 . . . . . . 64

5.2 .6 Magnetic energy and helicity budget . . . . . . . . . . . . . . . . . . 66

5.3 Small-scale dynamics . . . . . . . . . . . . . . . . . . . . . . . . 67

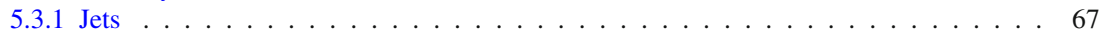

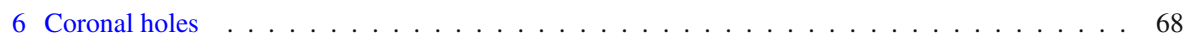

6.1 Properties of coronal holes . . . . . . . . . . . . . . . . . . . . 69

6.1.1 Properties of photospheric fields associated with coronal holes . . . . . . . . . . . . 69

6.1.2 Modeling of coronal-hole magnetic fields . . . . . . . . . . . . . . . . 70

6.2 Polar coronal holes . . . . . . . . . . . . . . . . . . . . . . . 71

6.2 .1 Magnetic flux in polar coronal holes . . . . . . . . . . . . . . . . . . . . 71

6.2.2 Comparison to the quiet-Sun magnetic field . . . . . . . . . . . . . . . . . . 71

6.2 .3 Polar plumes . . . . . . . . . . . . . . . . . . . . . 73

6.2 .4 Polar jets . . . . . . . . . . . . . . . . . . . . . . . . . . 74

6.2 .5 Contribution to the solar wind . . . . . . . . . . . . . . . . . . 74

6.3 Equatorial coronal holes . . . . . . . . . . . . . . . . . . . . . . 75

6.3 .1 Formation and evolution . . . . . . . . . . . . . . . . . . 75

6.3.2 Comparison with quiet-Sun magnetic fields . . . . . . . . . . . . . . . 76

6.3 .3 Contribution to the solar wind . . . . . . . . . . . . . . . . . . . 77

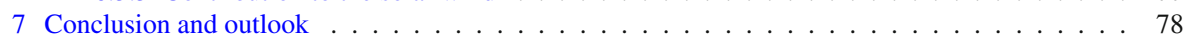

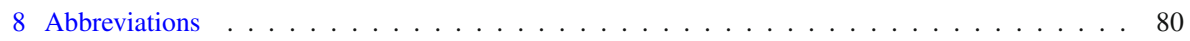

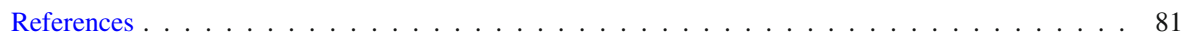

\section{Introduction}

To understand the physical processes in the solar interior, its atmosphere as well as the interplanetary environment (including "space weather" close to Earth), a detailed knowledge of the temporal and spatial properties of the magnetic field is essential. This is because the magnetic field is the link between everything, from the Sun's interior to the outer edges of our solar system. The magnetic field is created in the solar interior, can be measured with highest accuracy on the Sun's visible surface (the photosphere) and controls most physical processes in the solar atmosphere. Within this review, we aim to give an overview of the magnetic coupling from the solar surface to the Sun's upper atmosphere, with special emphasis on the structure and evolution of the coronal magnetic field. Magnetic features in the photosphere are discussed if they cause a coronal response. 
The techniques and challenges of measuring the magnetic field throughout the atmosphere are not discussed here, but are covered by earlier reviews (Raouafi 2005; White 2005; van Driel-Gesztelyi and Culhane 2009; Cargill 2009; Stenflo 2013). Outside the scope of this paper is the generation of the solar magnetic field by dynamo processes (for comprehensive reviews see, Ossendrijver 2003; Charbonneau 2010). For an in-depth discussion of the observational patterns resulting at photospheric levels from the dynamics in the Sun's convection zone, we refer to Zwaan (1987) and Stenflo (1994). We also do not discuss the role of the magnetic field and related physical processes far away from the Sun (beyond the solar corona) and its transport to those places. Here, we refer the interested reader to specialized reviews on the solar wind (Marsch 2006; Ofman 2010; Bruno and Carbone 2013), space weather (Schwenn 2006), and the heliospheric magnetic field (Owens and Forsyth 2013).

We start our review by giving an introduction to the most important magnetic aspects of the lower solar atmosphere, including the photosphere (Sect. 1.1), chromosphere (Sect. 1.2) and the corona (Sect. 1.4). The magnetic coupling between these layers is discussed in Sect. 1.3. An overview on the current most widely used local and global model approaches to assess the coronal magnetic field is given in Sect. 2. In the remaining sections, we provide more detailed descriptions of what we know to date about the coronal magnetic field's structure in different parts of the Sun's atmosphere, starting with the magnetic field on global scales (Sect. 3), in active and quiet-Sun regions (Sects. 4 and 5, respectively). Finally, we review the magnetic aspects of coronal holes in Sect. 6 and provide a summary and outlook in Sect. 7.

In most cases, we restrict ourselves to mention whether the discussed results were obtained from the analysis of directly measured magnetic fields or inferred from modeling. For further reading, we want to draw the reader's attention to classical overviews of the theoretical aspects of solar magnetism by Parker (1979) and Priest (1982, 2014), as well as previous descriptions dedicated to aspects of the magnetic properties of the Sun's magnetic field by Solanki et al. (2006). We also refer to Schrijver and Zwaan (2000) for a comparative work on the magnetic activity of the Sun and other stars.

Abbreviations used throughout this manuscript are defined in Appendix 8.

\subsection{Photosphere}

The photosphere contains the visible solar surface and vertically spans about $500 \mathrm{~km}$ of the solar atmosphere, where the temperature decreases from about $6,000 \mathrm{~K}$ at the bottom of the photosphere to about 4,000K (temperature minimum; Foukal 2004). In these layers, due to the momentum gained on its journey towards the surface, the convective material of the Sun's interior overshoots into the solar atmosphere, which is stable against thermal convection. Only after passing a distance comparable to the density scale height does it eventually turns over to form lanes of down flowing material (see reviews by Nordlund et al. 2009; Stein 2012). As a consequence, the photosphere reveals a granular pattern comprised of ascending warmer gas in the centers of the granules and descending cooler gas in the intergranular lanes separating them. In contrast to the layers below the solar surface, in the atmosphere the energy is dominantly transported by radiation rather than convection. 


\subsubsection{Magnetic flux emergence}

A significant part of the properties of the photospheric magnetic features is determined by the amount of magnetic flux carried by the $\Omega$-loops that rise through the convection zone towards the solar surface. The largest of these loops may form large bipolar ARs that harbor sunspots or sunspot groups (Durrant 1988). Large sunspots and sunspot groups have magnetic fluxes of $\approx 10^{21}$ and $10^{22} \mathrm{Mx}$, respectively (Priest 1982, 2014), and are responsible for a great part of the Sun's activity (see Sect. 4 for details). Much of the flux in ARs that is not in the form of sunspots is organized in magnetic concentrations (much) smaller than spots, either in the form of pores or, most commonly, magnetic elements. Magnetic pores, sunspot-like features that are characterized by the absence of a penumbra, carry fluxes of some $10^{20}$ to $10^{21} \mathrm{Mx}$ (Thomas and Weiss 2004; Sobotka et al. 2012). Magnetic elements within ARs carry fluxes of $10^{18}$ to $10^{20} \mathrm{Mx}$ (Abramenko and Longcope 2005). Note that it is unclear, however, whether the larger flux features observed by Abramenko and Longcope (2005) are indeed bright magnetic elements, or possibly darker features such as protopores.

Smaller rising $\Omega$-loops result in the formation of smaller ARs until a lower limit of roughly $10^{20} \mathrm{Mx}$. Below that we generally speak of "ephemeral regions" $\left(10^{18}\right.$ to $\left.10^{20} \mathrm{Mx}\right)$. Even smaller are the smallest so far resolved bipolar features, the internetwork magnetic loops (Martínez González et al. 2007; Centeno et al. 2007; Martínez González and Bellot Rubio 2009; Danilovic et al. 2010a) which emerge throughout the QS (although preferring a meso-scale pattern). They have fluxes of roughly $10^{16}$ to $10^{17} \mathrm{Mx}$ (Lin and Rimmele 1999) and display in general weak equipartition (that is, the magnetic energy density is similar to the kinetic energy density of the convective flows) intrinsic fields. Occasionally, these weak fields may be intensified due to a convective collapse (Parker 1978b; Spruit 1979). The latter amplifies the magnetic field in intergranular downflow regions due to the combined effect of enhanced cooling of the intergranular plasma (due to the transport of flux by the horizontal granular flows into this region) and the super-adiabatic stratification of the ambient plasma. In small flux concentrations, however, radiative energy exchange may be able to considerably slow down the cooling of the downflow material so that the collapse is prohibited and the gross part of this field remains relatively weak (see Venkatakrishnan 1986; Solanki et al. 1996; Grossmann-Doerth et al. 1998, and Sect. 5 for further details).

It is interesting to note that although each AR typically carries 100 times as much flux as an ephemeral region, the number of ephemeral regions appearing on the solar surface over a solar cycle outnumbers that of ARs by a factor of $10^{4}$, so that the ephemeral regions bring roughly 100 times more magnetic flux to the solar surface than ARs. Similarly, ephemeral regions carry roughly 100 times as much flux as a typical internetwork feature but all internetwork features appearing over a solar cycle together provide roughly 100 times more magnetic flux (Zirin 1987, and note that this is partly offset by the much lower lifetime of the smaller magnetic bipolar features). Altogether, the number of magnetic features with a certain amount of flux follows a power law distribution with an exponent of -1.85 (Parnell et al. 2009), which is close to -2.0 found by Harvey and Zwaan (1993). The latter means that, at any given time, small and large magnetic regions contribute a similar magnetic flux. 


\subsubsection{Spatial properties of magnetic features}

The different types of bipolar features have rather different properties. The ARs are largely restricted to the activity belts (i.e., within approximately $\pm 30^{\circ}$ around the solar equator; see Hale and Nicholson 1925). Their constituent sunspots are more or less E-W aligned with a certain tilt, with respect to the exact $\mathrm{E}-\mathrm{W}$ direction (corresponding to Joy's law; see Hale et al. 1919). This tilt increases with increasing latitude (Caligari et al. 1995; Li and Ulrich 2012) and seems to be inversely correlated to the strength of the upcoming solar cycle (Dasi-Espuig et al. 2010). Variation of the number of sunspots with time is often used as a measure of the solar cycle. Lifetimes of sunspots vary over a range of periods, with the larger ones living for months (Petrovay and van Driel-Gesztelyi 1997). ARs have been reported to have a tendency to emerge near existing ARs forming so-called active longitudes (Ivanov 2007), although there has been controversy regarding their reality (see Sect. 3 for a more detailed discussion).

Despite being preferentially concentrated around the activity belts (Harvey and Martin 1973; Martin 1988), ephemeral regions appear over a much larger fraction of the solar surface (Yang and Zhang 2014), indicating that they may be generated by a local rather than global dynamo process. Without observations of the poles, however, this claim is not tenable (see Sect. 6.2 for further details). They live for hours to days and display a tendency to align with the E-W orientation than ARs. They may even not have such a trend at all (Hagenaar et al. 2003; Yang and Zhang 2014). Their number varies much less over a solar cycle than that of ARs and there are inconsistent results regarding whether their number varies in phase or in anti-phase with the solar cycle (Martin and Harvey 1979; Martin 1988; Hagenaar et al. 2003).

Whereas the location of ARs and ephemeral regions are determined mainly by the latitudes and longitudes of emergence, the spatial distribution of other magnetic features, such as the magnetic network, is also influenced by the transport of magnetic flux at the solar surface by a variety of flows. The properties of the magnetic network change in the course of the solar cycle: around solar minimum it is weak and consists mainly of mixed polarities, except near the poles which are essentially unipolar regions (and with each pole having a different polarity). Around solar maximum, the mixedpolarity regions are augmented by large unipolar regions up to solar latitudes of about $60^{\circ}$ which are the decay products of old ARs. Finally, the internetwork fields appear all over the Sun, including also the interior of ARs. Individual internetwork elements live only for minutes to hours and they show no preference for any particular orientation (de Wijn et al. 2009, and references therein). They display no dependence on the solar cycle to the extent that can be tested so far (Bühler et al. 2013).

\subsubsection{Origin of internetwork fields}

There has been considerable debate concerning the origin of internetwork fields. One proposal regarding their origin is that they are either the consequence of recycling of magnetic flux from ephemeral regions, or are the result of convection acting upon ARs, tearing flux away and recycling it over time (Ploner et al. 2001). This basically implies that they are composed of flux produced by the global dynamo being one possibility and magnetic flux produced by a local dynamo being another (Vögler and Schüssler 
2007; Schüssler and Vögler 2008; Bühler et al. 2013; Stenflo 2012, and for a review see Martínez Pillet 2013). It is still an open question whether the quiet Sun's magnetic field is created mainly by the global dynamo or a local turbulent dynamo. One possibility to investigate this is the latitude dependence, where the global dynamo would likely lead to a significantly different distribution of quiet-Sun areas as a function of latitude, while the action of a local dynamo would not. Another approach is to trace quiet-Sun regions in time over a solar cycle. While a global dynamo would lead to a significant change as the cycle progresses, a local dynamo would not. This approach was applied by Bühler et al. (2013), based on circular and linear polarization signals measured with Hinode/SOT-SP during about half a solar cycle (during the years 2006 to 2012). No significant changes, in both linear and circular polarization, were found, in particular for magnetic features with a LOS magnetic flux of less than $10^{19} \mathrm{Mx}$. Thus, their results are favoring a local turbulent dynamo, at least for the creation of weak internetwork fields, and supporting what has been suspected in earlier studies (Sánchez Almeida 2003, and references therein and see also Sect. 6.2.2 for the importance of a local dynamo in the Sun's polar regions).

\subsubsection{Temporal evolution of the magnetic field}

The emergence of magnetic flux ropes from below the surface within ARs is usually followed by the growth and separation of the opposite polarity patches. Most commonly, loop footpoints move apart almost linearly with time (Centeno et al. 2007). But also more complex motions such as circular ones are possible, although only if the emerging loop possesses a writhe or a twist (Guglielmino et al. 2012). Then, physical long-term (López Fuentes et al. 2003) and apparent short-term rotational motions (López Fuentes et al. 2000; Luoni et al. 2011) of the opposite polarity patches are usually observed. And also apparent shearing and rotational motions have been noted (Gibson et al. 2004; Liu and Zhang 2006). (Note that whenever we speak of shearing without any specification, a horizontal motion, i.e., parallel to the solar surface, is referred to.) Sunspots also can show an apparent rotational motion around their center shortly after emergence. The related coronal magnetic loops (which magnetically connect the rotating sunspots) are often twisted and visible as sigmoid structures in coronal images (Brown et al. 2003). Coronal structures above rotating sunspots are also prone to cause flaring activity.

Once the $\Omega$-loops have emerged, the enhanced magnetic field at their footpoints (the magnetic patches) interacts with the convection in different ways (Schrijver et al. 1997). At the beginning, the magnetic field is generally approximately in equipartition with the flows (typically granular flows). At the solar surface, this corresponds to field strengths of 300 to $500 \mathrm{G}$. Once the field has emerged, it gets concentrated to form $\mathrm{kG}$ (kilogauss) features by its interaction with convective flows (Parker 1978a; Nagata et al. 2008; Danilovic et al. 2010b). Recent studies suggest that the concentration of the field can be followed by a weakening of the field and that this can cycle multiple times (Requerey et al. 2014). The flows also move the magnetic features around, causing each to carry out a random walk, although the exact nature of the motion can differ, depending on the location of the magnetic feature (Abramenko et al. 2011; Jafarzadeh et al. 2014a). 
(a)

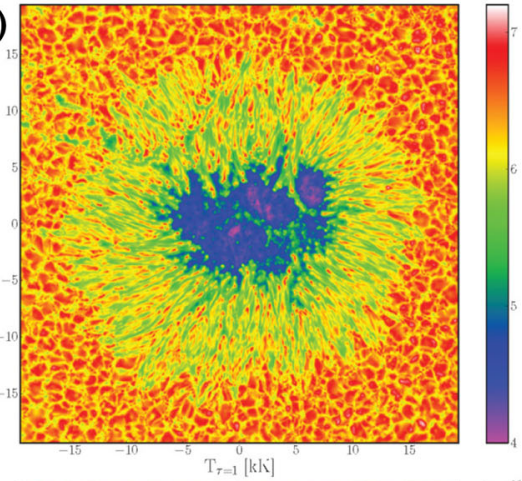

(b)

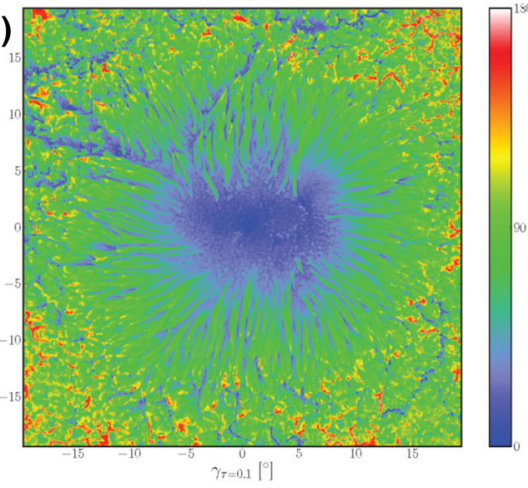

(c)

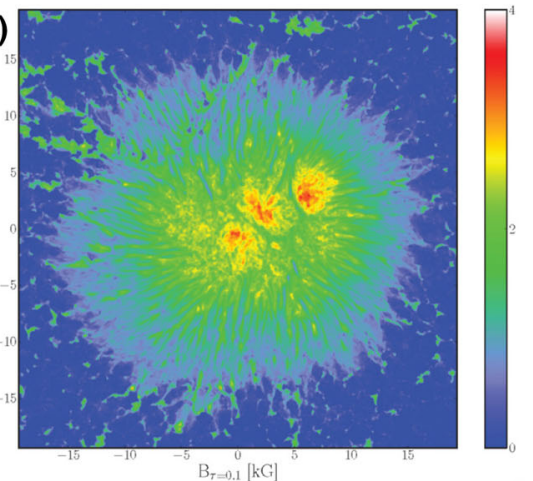

(d)

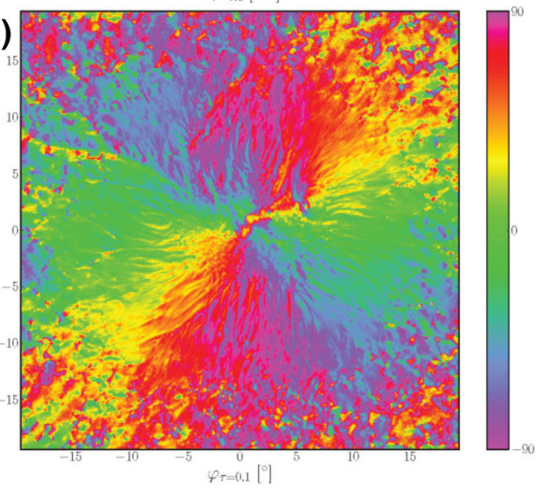

Fig. 1 Structure of the magnetic field and the temperature of a relatively symmetric sunspot recorded by the Hinode/SOT-SP on January 2007 which scanned the sunspot area from 12:36 UT to 13:00 UT. Plotted are maps of the a temperature, $\mathbf{b}$ field strength, $\mathbf{c}$ field inclination and $\mathbf{d}$ azimuth. The fine-scale structures of this sunspot have been analyzed by Tiwari et al. (2013) and van Noort et al. (2013)

The random walk of the magnetic patches, imposed by the convective motions, necessarily leads to the encounter of opposite polarity fields. These, in the case of smaller flux tubes, often do not correspond to the other footpoint of the original $\Omega$-loop, so that in larger ARs, a fair amount of cancelation takes place (Livi et al. 1985). When fields of same polarity meet, larger flux concentrations are (sometimes only temporarily) formed (Martin 1984). Only if there is enough magnetic flux of a single polarity present, then part of it coalesces into a sunspot. Proper sunspots consist of a central umbra and a surrounding penumbra (see Fig. 1). The latter is a filamentary structure of weaker, more horizontal magnetic field which surrounds the more vertically oriented stronger umbral magnetic field (Solanki 2003; Borrero and Ichimoto 2011, for reviews see). Typically, magnetic field strengths of about $1 \mathrm{kG}$ are found in penumbrae while the maximum umbral field strengths usually range between 2 and 4 kG (Title et al. 1993; Lites et al. 1993; Schad 2013). In extreme cases, values as large as $6 \mathrm{kG}$ have been reported (Livingston et al. 2006). Only recently, van Noort et al. (2013) reported $\approx 7 \mathrm{kG}$ in a sunspot, although surprisingly not in the umbral area but near the outer edge of the penumbra, in a strong downflow region. Sunspots have diameters between $3 \mathrm{Mm}$ (megameter) and $60 \mathrm{Mm}$ and live for a few hours to 
months (Bray and Loughhead 1964, and see also the review by Solanki 2003), with the lifetime being linearly correlated to the maximum area covered (Waldmeier 1955). Sunspots grow rapidly after their emergence, soon reach a maximum size and decay slowly afterward.

Sunspots are often preceded, accompanied and followed by "faculae" (called "plage" at chromospheric levels) which have a spatially averaged field strength of typically between 100 and $500 \mathrm{G}$ (Title et al. 1992) and are composed of magnetic elements of a range of sizes, with comparatively field-free or weak-field gas in between. Faculae tend to surround and generally outlive the sunspots by a significant amount. Consequently, an old AR is generally composed of faculae only which then decay and disperse to form enhanced network fields. The flux in ARs that is not in the form of sunspots is concentrated in either pores or magnetic elements. The properties of pores include diameters of some $\mathrm{Mm}$ and field strengths of 1 to $2 \mathrm{kG}$ (Thomas and Weiss 2004; Sobotka et al. 2012). Magnetic elements have diameters smaller than $\approx 350 \mathrm{~km}$ and exhibit field strengths of 1 to $2 \mathrm{kG}$ (Stenflo 1973; Rabin 1992; Rüedi et al. 1992). They are the chief magnetic constituents of faculae, which are bright (i.e., hotter than their surroundings) particularly in the mid-photosphere and above (see reviews by Solanki 1993; Solanki et al. 2006) and are present even in the internetwork (Lagg et al. 2010).

Sooner or later, larger magnetic features (e.g., sunspots) break up and dissolve, their fragments becoming subject to transport and distortion by the convective flows. The smallest and most dynamic convective elements in the QS are granules. Granules have typically diameters of $500 \mathrm{~km}$ to $1.5 \mathrm{Mm}$, a single turnover time of a few minutes and lifetimes of minutes (Nordlund et al. 2009; Zhou et al. 2010). Roughly, the turnover time is the time it takes for hot matter to be transported up through the solar surface, cooled there and transported down again in an intergranular lane, while the lifetime is the time over which a given granule maintains its identity (e.g., in a series of images of the solar surface). In the QS, the magnetic field is additionally swept to the edges of supergranular cells (for a review see Rieutord and Rincon 2010) with typical diameters of 20 to $30 \mathrm{Mm}$. This happens on a timescale of several hours and leads to the formation of a patchy magnetic network outlining the boundaries of the supergranular cells.

The transport of magnetic flux to the edges of the granular and supergranular cells leads to an enhancement of magnetic flux if the accumulated flux is of the same polarity. Only when the magnetic elements of opposite polarity meet, do they (partially) cancel. In fact, the most significant process of disappearance of magnetic flux appeared to be the cancelation of magnetic elements of opposite polarity (Livi et al. 1985). Wang et al. (1988) concluded that the flux cancelation occurs as a consequence of magnetic reconnection in or above the photosphere, which is likely due to the expansion of the field, so that the opposite polarities meet mainly in the upper photosphere (Cameron et al. 2011). However, a recent study of Lamb et al. (2013) suggests that at least in the QS, flux dispersal is the more common route by which magnetic elements are destroyed, although the exact physical process of flux removal could not be studied (dissipation at small spatial scales is likely to play a role). Another explanation for the apparent disappearance of magnetic flux is that the continuous buffeting of the magnetic flux concentrations leads to the fragmentation of some of the flux into entities whose lesser 
magnetic flux may then be below the detection threshold of a particular instrument (Berger and Title 1996).

\subsubsection{Relative importance of magnetic forces}

Typical values for the particle number density in the solar photosphere are in the order of $n \approx 10^{23} \mathrm{~m}^{-3}$ (at the temperature minimum; Foukal 2004). Typical quietSun and active-region magnetic field strengths cover the range $100 \mathrm{G}$ to $2 \mathrm{kG}$. As a consequence, the ratio of the plasma pressure to the magnetic pressure (usually referred to as plasma- $\beta$, or simply denoted by $\beta$ ) is in the order of 1 to 10 (when averaged over larger regions, Gary 2001). Note that a value of $\beta \gtrsim 1$ implies that the pressure exerted by the plasma is higher than that exerted by the magnetic field, i.e., that the plasma motion controls the dynamics (and the photosphere is therefore generally said to be "non force-free"). Locally, however, due to the evacuation of magnetic features values of $\beta<1$ are often found. Consequently plasma pressure forces might not be dominant everywhere in the photosphere. Sunspots and $\mathrm{kG}$ magnetic elements (for instance at supergranular boundaries) likely represent such exceptions (Priest 1982).

Solanki et al. (1993) found that in the layers of sunspots near the bottom of the photosphere, $\beta$ is likely above unity everywhere. It was found to drop from higher values in the umbral center and to reach $\beta \approx 1$ at the umbral boundary, followed by another increase towards the outer penumbral boundary. In contrast, Mathew et al. (2004), who used the same spectral lines as Solanki et al. (1993), presented a case where both, the entire umbra as well as the inner penumbra of a sunspot had a $\beta$ slightly below unity. More recently, Tiwari (2012) statistically addressed this topic using high-resolution magnetic field information for 19 sunspots. He found that in mid-photospheric layers most of the fine structures over most of the sunspot areas were nearly force-free with the tendency that umbral fields were less forced, while penumbral fields were more (see Fig. 2). This combination of large plasma- $\beta$ in a
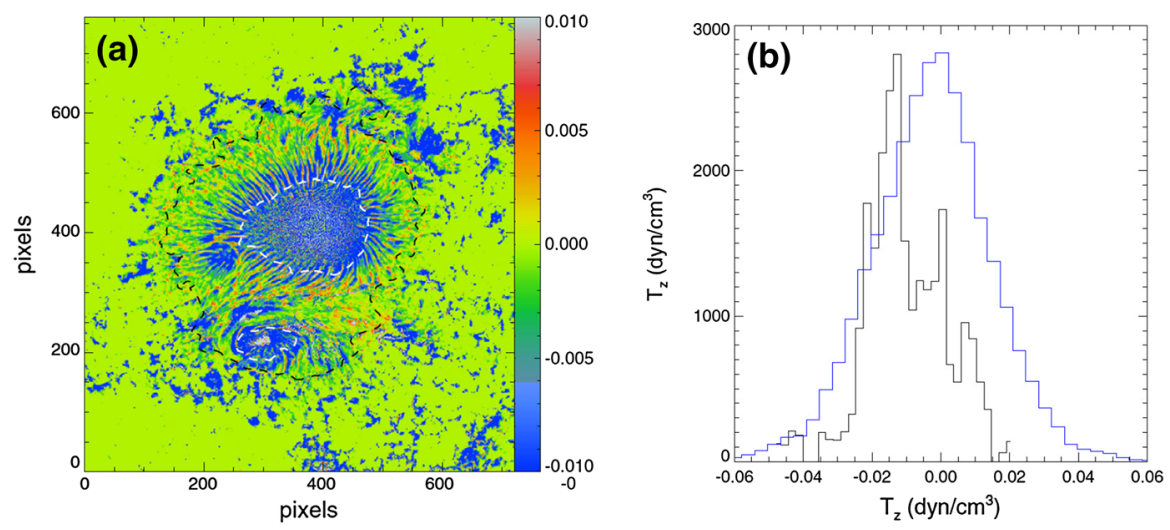

Fig. 2 a Map of the vertical tension force $\left(T_{z}\right)$ of an active-region sunspot. Gray and black dashed lines outline the boundaries of the umbra and penumbra, respectively. $T_{z}$ has high negative values at most places over the sunspot. b Histograms of $T_{z}$ in the umbra (black) and penumbra (blue). The histogram peak for the umbral field is shifted towards higher negative values, i.e., the umbral field is more force-free than that in the penumbra. (Adapted from Figure 2 of Tiwari (2012). (C) AAS. Reproduced with permission) 
spatially averaged sense and small values of $\beta$ locally has important consequences. A comparatively low $\beta$ inside strong-field features helps to explain why they maintain their identity for often considerable lengths of time. The high average $\beta$ implies that magnetic features as a whole, more or less passively, follow convective motions. This in turn explains that the magnetic field in the corona can become tangled and complex (see Sect. 4).

The relative importance of magnetic forces in entire ARs was also estimated. Metcalf et al. (1995) found a value of $\simeq 0.4$ for the net Lorentz force (i.e., the ratio of the total vertical Lorentz force and magnetic pressure force, integrated over the area of the considered AR) and concluded that the analyzed AR cannot be validly considered as to be force-free at a photospheric level. In contrast, Gary et al. (1987) found that another analyzed AR was indeed force-free, except for some, localized areas (areas for which flaring activity was noticed). Moon et al. (2002) analyzed the forces within three flare-productive ARs and found a median of $\approx 0.1$ for the net vertical Lorentz force and argued that the magnetic field at photospheric levels may not be as far from being force-free as commonly assumed. (See also Sect. 5.2.5, for a discussion on the force-freeness of quiet-Sun regions.)

The above compilation shows that the findings, so far, are not entirely conclusive regarding how close to being force-free the photospheric magnetic field really is, they rather show that the amount of forcing (by the gas) depends on the situation being considered. Therefore, special care is required when using photospheric vector magnetic field data as input for, e.g., coronal magnetic field models. Such modeling often relies on routine measurements of the magnetic field, which are to date predominantly performed at photospheric levels (see Sect. 2).

\subsection{Chromosphere}

The chromosphere lies on top of the photosphere with a thickness of about 1 to $2 \mathrm{Mm}$, starting from the temperature minimum in traditional one-dimensional model atmospheres. In reality, the chromosphere is far more complex and its thickness is likely to vary strongly from one horizontal location to another. Importantly, it should be thought of more as a temperature rather than a static height regime, with the temperatures increasing from the temperature minimum to $\approx 10^{4} \mathrm{~K}$ (Stix 2002). Sketches indicating the rich variety of phenomena in the chromosphere and its complexity have been presented in reviews by Wedemeyer-Böhm et al. (2009) and Rutten (2012). Just as the small-scale dynamics of the photosphere are dominated by granular convection, those of the chromosphere are dominated by waves. In internetwork regions these are mainly acoustic waves with a three-minute period, produced in the convection zone (for reviews see Rutten and Uitenbroek 1991; Carlsson and Stein 1997; Wedemeyer et al. 2004). But there is also mounting evidence of MHD waves in the chromospheric layers of magnetic structures (Hansteen et al. 2006; De Pontieu et al. 2007).

\subsubsection{Characteristic chromospheric magnetic structures}

The enhanced magnetic flux concentrations outlining the supergranular cells (the magnetic network) in the photosphere coincide with the bright network seen in chro- 
mospheric spectral lines (e.g., of CaII). The spatial agreement results from the fact that the magnetic features are nearly vertical (Martínez Pillet et al. 1997; Jafarzadeh et al. 2014b), which is the result of the large field strength of the photospheric $(\approx \mathrm{kG})$ flux tubes. Strong fields produce nearly evacuated structures which result in the flux tubes being buoyant (Parker 1955) and therefore cause a radial (i.e., vertical) orientation of the field. The vertical orientation is maintained also in the presence of horizontal granular flows (Schüssler 1984) which bend the magnetic elements (Steiner et al. 1996). The magnetic elements appear bright in chromospheric radiation and larger in size than in the photosphere (Gaizauskas 1985). Smaller magnetic features are brighter than their surroundings in photospheric radiation due to the vertical, evacuated structures being less opaque than their surroundings. As a consequence, the radiation from the flux tube's walls may penetrate deep into the thin flux tube's interior which then appears bright (Spruit 1976, and for reviews see Solanki 1993; Steiner 2007). To explain the enhanced brightness in the chromosphere, however, additional sources of heating, such as the dissipation of waves propagating along the field lines (Roberts and Ulmschneider 1997) are necessary.

Both in active-region plage and in the network, the $\mathrm{kG}$ magnetic field structures appear more diffuse in the chromosphere than in the photosphere (Jones 1985; Petrie and Patrikeeva 2009). While the photospheric field is mainly radially oriented, the chromospheric field expands in all directions forming a magnetic canopy (Giovanelli 1980; Jones and Giovanelli 1982), which is likely to be a natural consequence of the excess heating inside magnetic elements (Solanki and Steiner 1990). Choudhary et al. (2001) compared LOS chromospheric magnetic field as observed in the CaII $8542 \AA$ spectral line with a current-free magnetic field model. The latter was based on photospheric LOS magnetic field observations in the Fe I $8686 \AA$ spectral line. Analyzing 137 ARs, they found that the chromospheric observations were reproduced best by a current-free model field at a height of $\approx 800 \mathrm{~km}$ above the photosphere, in agreement with the expected formation height of the Ca II $8542 \AA$ line. Their results also suggested a decreasing correlation between the observed and modeled LOS magnetic field with increasing field strength, which they attributed to change of the spectral line's formation height in strong-field regions (although a real deviation from a potential configuration remains a possibility).

On larger (active-region) scales, often observed as dark elongated features in $\mathrm{H} \alpha$ $6563 \AA$ and He I $10830 \AA$ images are filaments ("prominences" when observed above the limb; for reviews see Labrosse et al. 2010; Mackay et al. 2010). Filaments straddle polarity inversion lines and typically exhibit heights of $\approx 50 \mathrm{Mm}$, lengths of $\approx 200 \mathrm{Mm}$ and a thickness of a few Mm (Stix 2002). They are involved in many eruptive processes ("eruptive" filaments), but outside of ARs often persists for a long time in the QS ("quiescent" filaments). As suggested by the name, active-region filaments concentrate around the activity belt, while quiescent filaments can be located everywhere on the Sun. In principle, they are thought to be comparatively cool $\left(T \lesssim 10^{4} \mathrm{~K}\right)$ chromospheric material suspended in the corona, sustained by the geometry of the magnetic field. Early investigations of large samples of polar prominences (quiescent as well as eruptive) mainly based on Hanle effect measurements, revealed characteristic longitudinal field strengths in the order of 1 to 10 G (Leroy 1977; Leroy et al. 1983; Athay et al. 1983). For active-region filaments, the interpretation of the Zeeman effect 
revealed strengths of some $100 \mathrm{G}$ to $1 \mathrm{kG}$ for the vertical as well as horizontal field (Lites 2005; Kuckein et al. 2012; Xu et al. 2012). Xu et al. (2012) were furthermore able to trace the photospheric and chromospheric signatures of the same active-region filament, and detected differing morphologies. This led them to suggest that an emerging magnetic flux rope may, besides sustaining filament material at low atmospheric heights (upper photosphere to low chromosphere), at the same time be able to store plasma at the top part of the flux rope, i.e., at greater (mid chromospheric) heights.

On smaller scales, around sunspots, a radially outward-directed filamentary pattern is observed, persisting for hours to days, the sum of which is called the (chromospheric) "super-penumbra" (Hale 1908a). These structures are seen in almost all chromospheric spectral lines, including H $\alpha 6563 \AA$, Ca II $8542 \AA$ and Ly $\alpha 1216 \AA$ and, though more rarely, also in CaII H and K (see Pietarila et al. 2009, and references therein). A common assumption is that these chromospheric "fibrils" outline the direction of closed magnetic field structures in the upper photosphere and chromosphere, linking the spot with the surrounding flux of opposite polarity (Nakagawa et al. 1971; Woodard and Chae 1999), allowing a mass flow away from the spot (Evershed 1909) or into the spot ("inverse Evershed effect"; St. John 1913, and for a review see Solanki 2003). In a similar fashion, fibrils (seen, e.g., in $\mathrm{H} \alpha$ ) are thought to connect opposite polarity magnetic flux elements in the QS (Reardon et al. 2011; Beck et al. 2014), although some fibrils may follow the chromospheric part of magnetic field lines that continue into the corona (see also Sect. 5). The fibril pattern around sunspots is often observed to be oriented radially outwards and forming whirls which exhibit rotation patterns specific to the hemisphere where they are observed (Hale 1908b; Richardson 1941; Peter 1996). Vecchio et al. (2007) underlined the likeliness of fibril-like structures seen in Ca II $8542 \AA$ images to outline the canopy at chromospheric levels. The fibrils are thought to follow the canopy magnetic field of sunspot super-penumbrae, whose base rises slowly from the edge of the spots as one moves radially outward alongside a decreasing magnetic field strength (Giovanelli 1980; Giovanelli and Jones 1982; Solanki et al. 1994, and see also Sect. 1.3.1).

\subsubsection{Indirect tracing of chromospheric fields}

Woodard and Chae (1999) investigated the non-potentiality of fibril structures in the QS. They performed a comparison of field lines from a potential field model with fibrils observed in $\mathrm{H} \alpha 6563 \AA$. They found, under the assumption that the fibrils trace magnetic field lines, that the observed fibril structure aligns well with the magnetic field model in some places, but not in others (see Fig. 3a). They concluded from this finding that the quiet Sun's chromospheric magnetic field is far from a potential state (i.e., it carries currents on small scales). This interpretation has been tested for activeregion fibrils by Jing et al. (2011) who based their study on a potential magnetic field model starting from chromospheric magnetograms. Again it was found that in some places the modeled horizontal field agrees well with the segmented fibril orientation but not in other places (Fig. 3b). It appeared that there is a link between the horizontal shear of the involved field and the mismatch between model and observation: the higher the shear of the observed chromospheric magnetic field structures, the lower the agreement with a potential magnetic field model. Consequently, potential field 
(a)

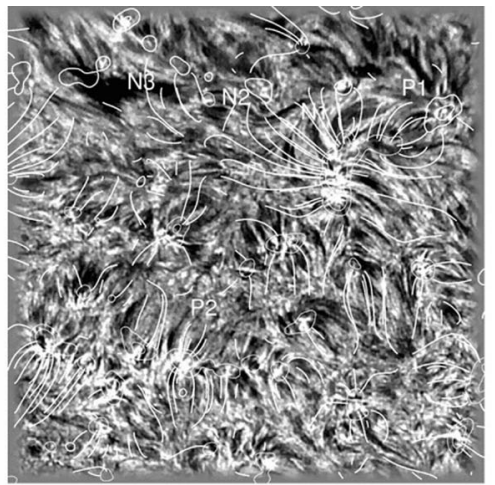

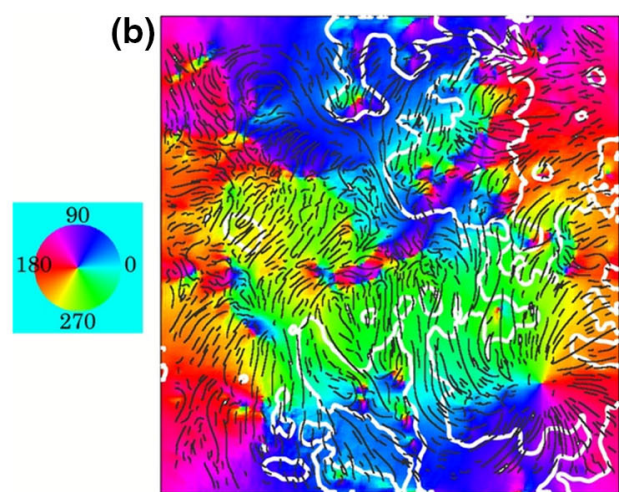

Fig. 3 a Comparison of $\mathrm{H} \alpha$ fibrils (observed at $6563 \AA$ A; gray-scale background) with projections of potential magnetic field lines over-plotted. The FOV spans roughly $300^{\prime \prime} \times 300^{\prime \prime}$. Except for some local areas denoted as P1 and P2 (upper right and lower middle part of the image, respectively), poor agreement between filed and the direction of fibrils is recognized in particular around N1, N2, and N3 in the upper mid to left part of the frame. (Figure 2 of Woodard and Chae 1999. With kind permission from Springer Science and Business Media.) b Comparison of $\mathrm{H} \alpha$ fibrils (at $6563 \AA$; black curves) with the chromospheric potential magnetic field azimuth, counted counter-clockwise from $0^{\circ}$ at solar west. The white contours outline magnetic PILs. The FOV is roughly $254^{\prime \prime} \times 264^{\prime \prime}$. Good agreement is found between the potential field azimuth and the fibril orientation in some places, while a clear deviation of the two direction is seen in others. (Adapted from Figure 5 of Jing et al. 2011. ( ) AAS. Reproduced with permission)

models, either based on photospheric or chromospheric magnetic field data, can in general not be assumed to adequately reproduce the (chromospheric) magnetic field, assuming that fibrils indeed outline the orientation of the chromospheric magnetic field.

An alternative interpretation of the results of Woodard and Chae (1999) and Jing et al. (2011) is that fibrils do not outline the orientation of the chromospheric magnetic field. This, however, is in direct contrast with the results of recent numerical simulations which suggested that $\mathrm{H} \alpha$ fibrils are visible manifestations of high-density ridges aligned with the magnetic field (Leenaarts et al. 2012), thus serving as an indirect tracer of the vertical-to-horizontal transition of the magnetic field orientation around magnetic flux concentrations. This was addressed by de la Cruz Rodríguez and Socas-Navarro (2011) who compared the observed orientation of fibrils in Ca II $8542 \AA$ images to the chromospheric magnetic field vector, inferred from observed polarization signals originating from the same spectral line. They found that most of the fibrils in the surrounding of a penumbral boundary nicely followed the magnetic field direction but also recorded a significant mismatch for a considerable number of fibrils (Fig. 4a). They also noted a too rapid decrease of the linear polarization signal when moving out of the penumbral area, if the fibril pattern indeed was to outline the super-penumbral field direction. The rapid decrease of the linear polarization signal, however, may be attributed to the height of the canopy base relative to the formation height of the spectral line. This was re-addressed recently by Schad et al. (2013) who, in contrast to de la Cruz Rodríguez and Socas-Navarro (2011), found a clear coincidence of the projected direction of super-penumbral fibrils and the inferred magnetic field (to within $\pm 10^{\circ}$ ) using He I $10830 \AA$ A observations. They detected a notable change of 
(a)

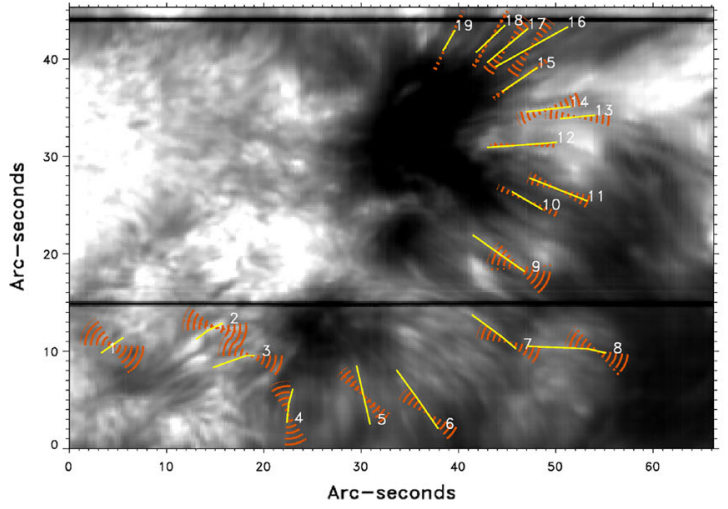

(b)

(c) Azimuth, $\chi_{B}$ (SOLAR) [deg]

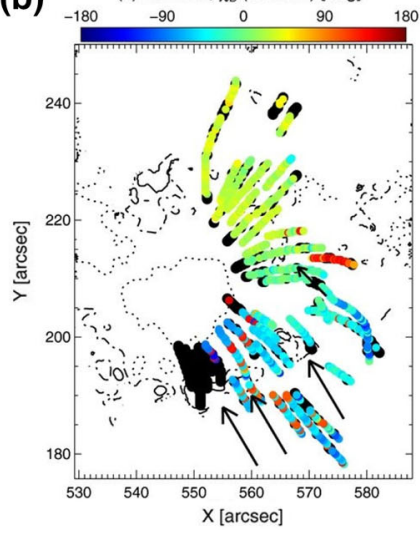

Fig. 4 a Comparison of visually determined directions (yellow lines) of Ca II fibrils (observed at $8542 \AA$; gray-scale background) to the magnetic field azimuth compatible with linear polarization signals (orange cones). While the fibril orientation is picked up by the reconstructed horizontal magnetic field orientation for a fair number of fibrils (e.g., fibrils 9 to 19), it is only poorly recovered for others (e.g., fibrils 1 to 5). (Figure 1 of de la Cruz Rodríguez and Socas-Navarro 2011. Reproduced with permission from Astronomy and Astrophysics, (C) ESO.) b Spatial map of the magnetic field azimuth along selected super-penumbral fibrils, inferred from He I at $10830 \AA$ observations. The dotted and dot-dashed contours indicate a photospheric magnetic field inclination of $135^{\circ}$ and $90^{\circ}$, respectively. Black dots mark severe deviations between the inferred and observed fibril orientation. They are restricted to where fibrils turn to their photospheric rooting points. (Adapted from Figure 10 of Schad et al. 2013. (C) AAS. Reproduced with permission)

the inclination only close to where the fibrils turn towards their rooting point in the sunspot (Fig. 4b). Moreover, based on their findings, they explicitly support schemes which propose the inclusion of the spatial information delivered by chromospheric fibril observations to increase the success of force-free coronal magnetic field models. Such proposed schemes use the fibril information to increase the match between the modeled and observed horizontal field at chromospheric heights (where the magnetic field vector is not routinely measured; see Wiegelmann et al. 2005a, 2008; Yamamoto and Kusano 2012).

\subsubsection{Plasma- $\beta$ in the chromosphere}

Density and temperature are heavily structured in the highly dynamic chromospheric environment so that the relative strength of the plasma pressure and magnetic forces also varies strongly with position, at a given height. The height at which the magnetic forces start to dominate over others (i.e., where $\beta \ll 1$ ) is expected to be strongly corrugated relative to the solar surface. In the QS, that height is expected to vary between $\approx 800 \mathrm{~km}$ and $1.6 \mathrm{Mm}$ above the photosphere (Rosenthal et al. 2002). In ARs, this height is likely to be lower, as shown by Metcalf et al. (1995). They used chromospheric vector magnetic field measurements inferred from observations in the NaI $5896 \AA$ spectral line to test the relative contribution of the plasma pressure and magnetic forces in an AR. They found that the atmosphere above that AR could be considered to be force-free from $\approx 400 \mathrm{~km}$ above the solar surface. Gary (2001) was 


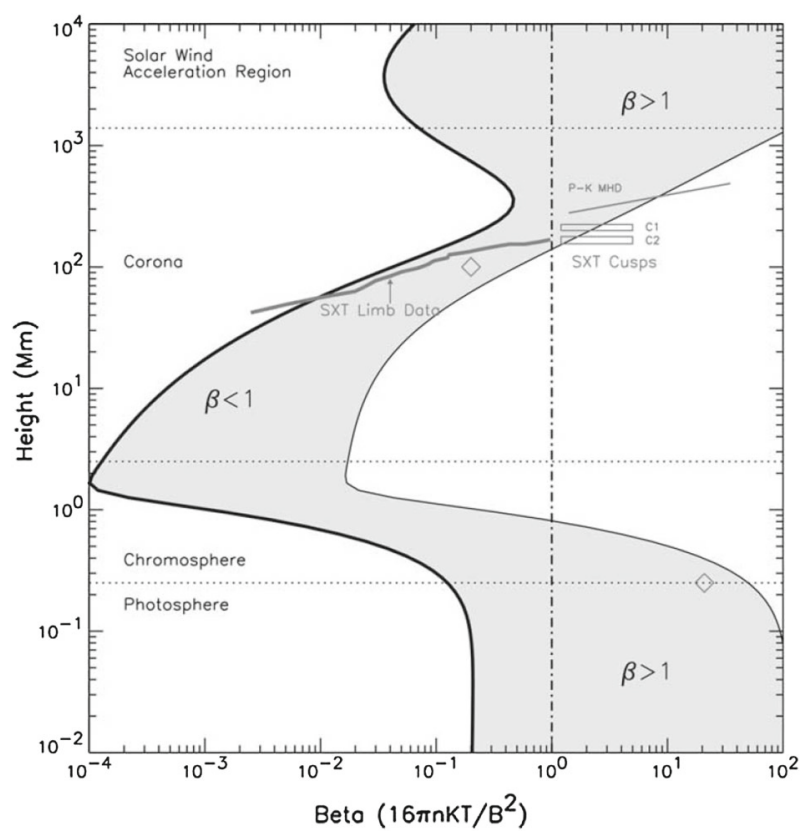

Fig. 5 Distribution of the plasma- $\beta$ as a function of height above an AR. The shaded area represents the vertical run of $\beta$ for open and closed fields originating between a sunspot (represented by the thin solid line) and a plage region (represented by the thick solid line). $\beta$ becomes only small $\left(\simeq 10^{-2}\right)$ at heights above the upper chromosphere and mid corona. Note that this low- $\beta$ region is sandwiched between high- $\beta$ regions (the photosphere and low to mid chromosphere below as well as the upper corona and solar wind acceleration region above). (Figure 3 from Gary 2001. With kind permission from Springer Science and Business Media)

able to confirm that finding by combining a plasma pressure and magnetic field model to estimate the pattern of interchanging dominance of plasma and magnetic pressure with height in the solar atmosphere (see Fig. 5). He concluded that the magnetic forces above sunspots should start to dominate from relatively low heights ( $\gtrsim 400 \mathrm{~km}$ above the photosphere). Above plage regions, the model results suggest this to be true from $\gtrsim 800 \mathrm{~km}$ above a photospheric level upwards. In summary, ARs can be considered to be force-free in most of the chromosphere (in contrast to quiet-Sun areas; see Sect. 5.2.5).

1.3 Magnetic coupling from the lower solar atmosphere to the corona

\subsubsection{Magnetic canopy}

At photospheric levels, only a small fraction of the solar surface is occupied by strong magnetic field $(\lesssim 5 \%)$. In contrast to that, the coronal magnetic field fills the entire coronal volume and is distributed relatively uniform in strength (although not in orientation). Consequently, the photospheric field must spread out with increasing height in the solar atmosphere. The magnetic field expands until it either turns over and returns 


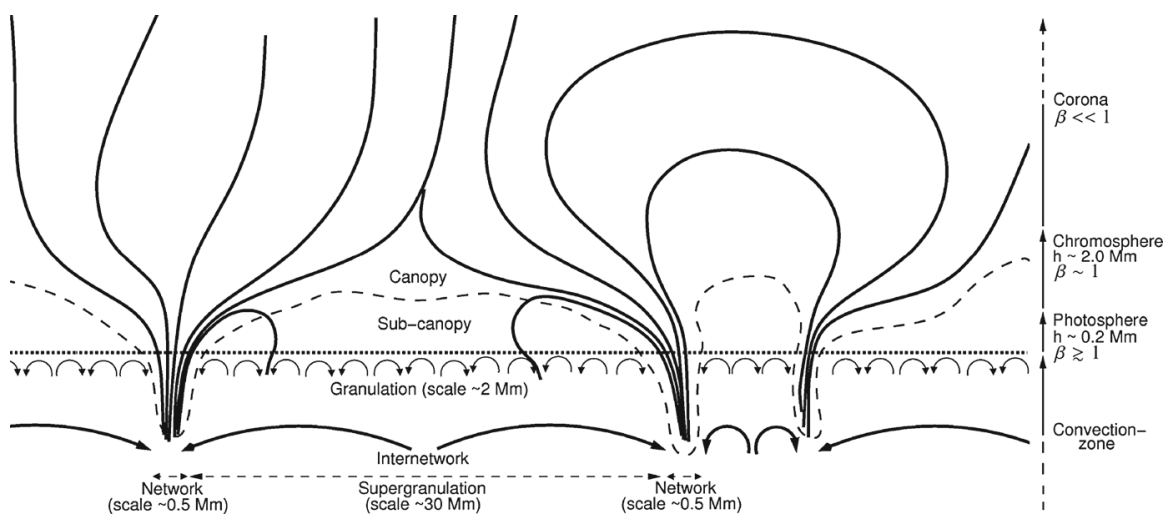

Fig. 6 Sketch of the quiet-Sun magnetic field structure in a vertical cross section through the atmospheric layers of the Sun. Swept to the edges of supergranular cells by large-scale convective flows (thick, large arrows at bottom), intense magnetic network elements or sunspots form. Small-scale convective flows (thin, small arrows below dotted horizontal line representing the photosphere) result in the photospheric granular pattern. The magnetic field lines (solid lines) expand at chromospheric heights and form the nearly horizontal magnetic canopy (dashed line). (Adapted from Judge 2006; Wedemeyer-Böhm et al. 2009)

to connect back to the photosphere or it meets the expanding field of the neighboring flux tubes. It then forms a "magnetic canopy", i.e., a base almost parallel to the solar surface and overlying a nearly field-free atmosphere (see Fig. 6). For a comprehensive review of the current picture of the magnetic coupling of the photosphere, chromosphere and transition region to the corona, we refer to Wedemeyer-Böhm et al. (2009) and restrict ourselves to a brief summary here. Estimates for the merging height of photospheric flux tubes range from some $100 \mathrm{~km}$ for active region to $\approx 1 \mathrm{Mm}$ for quiet-Sun magnetic fields (Spruit 1981; Giovanelli and Jones 1982; Roberts 1990). (Note that these estimates essentially depend on the filling factor, i.e., whether the considered region exhibits a high or low mean magnetic field strength.)

The expansion of the magnetic field with height is a consequence of the small gas-pressure scale height $(\approx 100 \mathrm{~km}$ in non-magnetic regions; Durrant 1988). From the lateral pressure balance follows that the field strength must rapidly decrease with height. (Remember that lateral pressure balance requires the gas pressure inside a flux tube to be lower than outside it.) With increasing height, the magnetic field strength drops due to the falloff of the gas pressure and flux conservation implies that the magnetic field must spread out, i.e., the extension of the magnetic structures must increase rapidly. Since magnetic features are hotter than their surroundings in the middle/upper photosphere and chromosphere, the internal gas pressure drops more slowly with height than the external gas pressure. As a consequence, at certain heights, the internal pressure force exceeds the external. This removes the lateral confinement of the magnetic structures and allows the structures to expand unhindered, until it hits field from another photospheric source. This implies a significant horizontal component of the field over a large part of the volume (the canopy; Solanki and Steiner 1990; Bray et al. 1991). The different merging heights thus depend, besides on the distance between neighboring magnetic features, on the temperature difference between the magnetic field structures and their surroundings, causing successively lower canopy heights for 
increasing temperature differences (see chapter 5 of Aschwanden 2005). Above this merging height, the magnetic field becomes increasingly homogeneous. Generally, the field of a magnetic element is seen to be shaped roughly like a wine glass. The direction of the field then depends on the structure of the magnetic field in its surroundings and the connectivity of the field lines on a larger scale (i.e., whether they are closed or open and where they return to the solar surface).

In plage regions, the flux tubes merge already in the mid to upper photosphere, so that the atmosphere above is almost fully magnetic (Buente et al. 1993). Model results suggested that, in the QS and in CHs where magnetic features are further apart, this base is located somewhere in the lower chromosphere (Gabriel 1976; Jones and Giovanelli 1982; Solanki and Steiner 1990; Solanki et al. 1991). Quite some time after the first speculations on the height of canopy-type magnetic fields, observational evidence for the merging heights in plage of in the order of several hundreds of $\mathrm{km}$ has been delivered (Steiner and Pizzo 1989; Guenther and Mattig 1991; Bruls and Solanki 1995). Rosenthal et al. (2002) performed numerical simulations of the propagation of waves through a model atmosphere, resembling properties of the chromospheric network and internetwork, and found the canopy height to vary between $\approx 800 \mathrm{~km}$ and 1.6 Mm above the base of the photosphere. However, a considerable number of findings, especially in the QS, led to serious doubts upon the reality of a large-scale, undisturbed magnetic canopy there (for details see Sect. 5.2.1).

Even though some aspects of the magnetic canopy, especially in the QS are still to be elaborated further, its basic nature seems clear: it is not a simple, rigid structure and also not at a constant height in the solar atmosphere. Instead, its shape and height are different for regions on the Sun with different amounts of magnetic flux and it also varies with time. Above the canopy, the coronal volume is filled more or less uniformly with magnetic field.

\subsection{Corona}

\subsubsection{Transition region and coronal base}

The corona is to be thought of as a temperature regime, covering a few times $10^{5} \mathrm{~K}$ in open field regions (such as CHs; see Sect. 6), $\approx 1$ to 2 MK (megakelvin) in the predominantly closed field of the quiet-Sun corona, and up to 2 to $6 \mathrm{MK}$ in ARs (see chapter 1 of Aschwanden 2005). It even can briefly reach values of 10 to $20 \mathrm{MK}$ during strong flares. It spans the atmospheric layers between the transition region (within which the temperatures increase from $\approx 10^{4} \mathrm{~K}$ to $\approx 1 \mathrm{MK}$ ) and the height where the solar wind is accelerated, i.e., spanning several hundreds of $\mathrm{Mm}$ in height (Gary 2001). The very narrow transition region not only bridges a large difference in temperature, but also separates the dilute coronal plasma (with number densities of $\left.n \lesssim 10^{12} \mathrm{~m}^{-3}\right)$ from the dense $\left(n \gtrsim 10^{16} \mathrm{~m}^{-3}\right)$ chromosphere (see chapter 1 of Aschwanden 2005). The base of the corona is not to be thought of as a horizontal layer somewhere above the solar surface. As the thickness of the chromosphere beneath varies, so does the height of the coronal base above the solar surface (see Sect. 1.3 for details). 


\subsubsection{Morphology of coronal magnetic fields}

Two very distinct magnetic configurations are present in the corona. The field is either arranged in the form of closed loops of enhanced emission, or in the form of open field lines seemingly not connecting back to the solar surface (Schrijver et al. 1999; Solanki et al. 2006). Arcades (ensembles) of bright coronal loops connect regions of opposite magnetic polarity on the solar surface and are often, but not necessarily always, rooted in an AR. Large-scale loop systems (sometimes exhibiting sigmoidal shapes) are often found to connect neighboring ARs and/or ARs with their quiet Sun surrounding (Strong 1994). Following Reale (2010), the observed coronal loop systems span a wide range of length scales, from a few Mm (bright points) up to giant arches which may span $1 \mathrm{Gm}$ (gigameter). Several loop arcades neighboring each other are often found in magnetically complex ARs and often host eruptive processes such as flares or CMEs (see Sect. 4). Therefore, in the majority of cases, bright coronal loops (see Sect. 4.1 for more details) are concentrated around the activity belts.

Most of the quiet-Sun magnetic fields (see Sect. 5) that reaches the corona is rooted in the magnetic network. At greater heights, they fan out to form funnels and to fill the coronal volume above (Gabriel 1976; Dowdy et al. 1986). Along the open field structures, plasma is efficiently transported outwards, which allows charged particles to escape from the solar atmosphere. Especially during solar activity minimum, open magnetic flux is concentrated around the poles, causing depleted regions which emit less than their surrounding temperatures above $1 \mathrm{MK}$ and consequently appear dark in coronal images (therefore termed "coronal holes"; see Sect. 6). At lower latitudes, the coronal structure is dominated by "helmet streamers" and "pseudo streamers", extending out to several solar radii in height (Schwenn 2006). Helmet and pseudo streamers are visible as enhanced emission in the form of a cusp above the limb, bridging the space between open fields of opposite and same polarity, respectively (see Pneuman and Kopp 1971; Wang et al. 2007).

\section{Magnetic field modeling}

The solar magnetic field is routinely measured mainly in the photosphere, whereas direct measurements in the higher solar atmosphere are available for individual cases. If the 3D magnetic field vector in the chromosphere and corona were to be measured routinely with high accuracy, cadence and resolution, indirect modeling approaches (as discussed in the following sections) would not be required. Since this is not yet the case (see Sect. 2.1), modeling approaches of different sophistication have been developed with the aim of computing the magnetic field in the upper solar atmosphere, generally starting from measurements made in the lower atmosphere.

One possibility is to use the longitudinal photospheric magnetic field component, or the measured full magnetic field vector (if available) as boundary condition for forcefree magnetic field reconstruction techniques. This is possible since the solar corona is almost force-free, because the magnetic pressure is several orders of magnitude higher than the plasma pressure. This allows neglecting non-magnetic forces to lowest order and applying such methods (see Sect. 2.2). Because these models are snapshots and 
assume stationarity and stability of the coronal magnetic field configuration, they are not to be used for modeling of dynamic features (such as CMEs, flares or eruptive prominences). Moreover, these models do not provide a self-consistent description of the coronal plasma. Time-dependent simulations are required for these aims, usually within the MHD approach (see Sect. 2.3). Full MHD models (see Sect. 2.3.1) are both theoretically and observationally very challenging because plasma and magnetic field have to be modeled self-consistently.

Complementary to these numerical approaches one can use the fact that the emitting coronal plasma (as visible in coronal images; see Sect. 4.1) is frozen into the magnetic field and consequently the coronal loops visible in the images outline magnetic field structures. Therefore, coronal images can be used to identify the $3 \mathrm{D}$ shape of the magnetic field structures when images from multiple viewpoints exist (e.g., from the STEREO-spacecrafts, SOHO or SDO). A 3D reconstruction of structures seen above the solar limb can be performed by stereoscopic and tomographic methods (see Sects. 2.4.1 and 2.4.2, respectively). Coronal images are also frequently used to validate the results of coronal magnetic field models. In some cases, time sequences of coronal images show oscillating coronal loops, which allow estimating the coronal magnetic field strength by coronal seismology (see Sect. 2.4.3).

The main aim of this section is to give a short overview of the methods for deriving the 3D magnetic field structure of the upper solar atmosphere (although we start this section with a short review of direct measurements of chromospheric and coronal magnetic fields). We refer to specialized reviews and the primary literature for further details. Outside the scope of the present review are methods of the interaction of the convection zone with the solar atmosphere by flux emergence (the interested reader can find a recent review on the theory of flux emergence in Cheung and Isobe 2014). Methods to analyze the 3D coronal magnetic field topology are described in Sect. 4.2 and also in a review by Longcope (2005).

\subsection{Direct coronal magnetic field measurements}

Direct measurements of the solar magnetic field are an important tool for understanding the magnetic field in the upper solar atmosphere. Here, we briefly introduce the most important methods for measuring the chromospheric and coronal magnetic field directly. The difficulties of performing such measurements are only briefly touched upon here (for details see Raouafi 2005; White 2005; van Driel-Gesztelyi and Culhane 2009; Cargill 2009). Thanks to instrumentation, e.g., the ground based NSO/DKIST (planned to become operational in 2019), together with powerful inversion techniques, coronal field measurements might become a prosperous method in future.

\subsubsection{Chromospheric magnetic field measurements in the infrared}

Infrared lines have been used to derive the magnetic field vector near the coronal base in the upper chromosphere. Initial measurements of the LOS magnetic field were performed by Harvey and Hall (1971), Rüedi et al. (1995) and Penn and Kuhn (1995) and the first vector magnetic field measurement by Rüedi et al. (1996). Solanki et al. 
(2003) applied the same method using the He I 10830 Å line, which is optically thin. Consequently, the measurements are related to different formation heights, following the fluctuating height of the coronal base. The authors managed the 3D structure of the chromospheric loops to be reconstructed by applying the following criteria. If a randomly selected pixel matches in field strength and direction of the two neighboring pixels, then the radiation is assumed to originate from the same loop. Because the full magnetic field vector is inferred, this allows to reconstruct the loop in 3D, with the additional constraint that the field strength decreases with height. The 3D structure deduced for the emerging loops was questioned by Judge (2009) but it was later shown by Merenda et al. (2011) that the proposed geometry provided a better representation to the data than the flat alternative proposed by Judge (2009). Simultaneously with these chromospheric measurements, the photospheric field vector was measured as well, and extrapolated into the chromosphere using force-free modeling techniques (see Sect. 2.2), where the NLFF model was found to agree best with the chromospheric observations (for details see Wiegelmann et al. 2005b).

\subsubsection{Coronal magnetic field measurements in infrared}

Coronal measurements in the infrared are possible from the ground with a coronagraph, or with instruments from space. An overview on some aspects of the usage of infrared lines to measure the coronal magnetic field can be found in Penn (2014). This review also gives a detailed discussion of advantages and disadvantages of using infrared lines in general (not restricted to coronal magnetic fields). More than ten coronal lines in the infrared have been identified and some of them are magnetically sensitive. The Fe XIII $10750 \AA$ line, for instance, has been used to measure the Stokes vector in the corona, which in principle would allow determining the magnetic field vector by an inversion. A general problem with coronal observations is, however, that due to the optically thin coronal plasma, any recorded radiation form the corona is integrated over the LOS. This naturally complicates the interpretation of the measurements, so that to derive the spatially resolved coronal magnetic field vector in $3 \mathrm{D}$, measurements from multiple viewpoints are necessary. The situation has some similarities with deriving the coronal density by a tomographic inversion (see Sect. 2.4).

\subsubsection{Coronal magnetic field measurements at radio wavelengths}

Radio signatures emitted from the active-region corona, currently represent the most widely used direct measure of the magnetic field. Because they are produced only in specific circumstances when electrons are guided by a magnetic field, they allow the reconstruction of the magnetic field strength in the corona (White et al. 1991; Schmelz et al. 1994; White and Kundu 1997; Brosius et al. 1997; Lee et al. 1998, 1999). Note that hard X-ray emission often goes hand in hand with radio emission since the efficient emission of both requires electron energies of $\gtrsim 10 \mathrm{keV}$ (see Fig. 7 and the review by White et al. 2011). Information on the height of the on-disk radio source in the corona is not accessible through such measurements, except occasionally for coronal structures at different heights above the solar limb using near infrared wavelengths (Arnaud 1982a, b; Lin et al. 2000, 2004) or radio observations (Brosius 

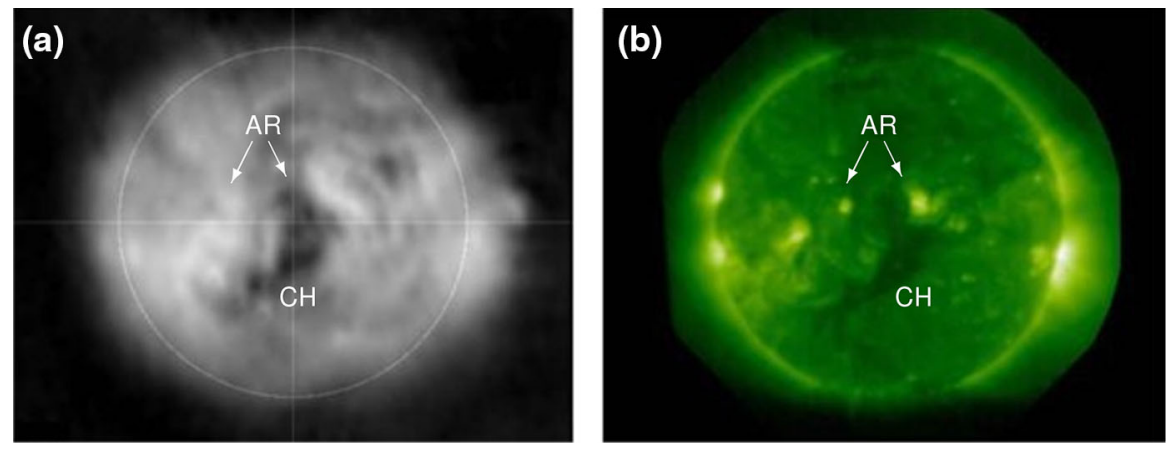

Fig. 7 a Full-disk NRH radio emission observed on June 27, 2004 at $432 \mathrm{MHz}$ and b GOES/SXI X-ray emission. The close relationship between the radio and X-ray emission can be seen in the form of bright emission around ARs and minimal emission in CHs. (Adapted from Figure 5 of Mercier and Chambe 2012. Reproduced with permission from Astronomy and Astrophysics, (C ESO)

and White 2006). For on-disk measurements, the lacking height information may be compensated by (force-free) magnetic field modeling of the coronal structure, starting from photospheric magnetograms (Liu and Lin 2008; Bogod et al. 2012; Kaltman et al. 2012). Furthermore, radio maps can be used also for a stereoscopic $3 \mathrm{D}$ reconstruction (see Sect. 2.4.1).

\subsection{Force-free modeling from photospheric measurements}

The solar magnetic field vector is measured routinely with high accuracy only in the photosphere, e.g., by SDO/HMI at a constant resolution of $1^{\prime \prime}$ over the whole solar disk. Under reasonable assumptions, we can extrapolate these photospheric measurements into the higher solar atmosphere, where direct magnetic field measurements are more challenging (see Sect. 2.1). So, which assumptions are reasonable in the solar atmosphere? A key to answer this question is the comparison of magnetic and nonmagnetic forces and in particular the plasma- $\beta$. While the plasma- $\beta$ is around unity in the photosphere it becomes very small (about $10^{-4}$ to $10^{-2}$ ) in the corona (at least in ARs; see Fig. 5 and Sect. 1.2.3 for details). Consequently, non-magnetic forces can be neglected in the low $\beta$ corona, and the coronal magnetic field can be modeled as a force-free field (the Lorentz-force vanishes). The electric current density,

$$
\mathbf{j}=\frac{1}{\mu_{0}} \nabla \times \mathbf{B},
$$

has either to be parallel or anti-parallel to the magnetic field, leading to

$$
\nabla \times \mathbf{B}=\alpha \mathbf{B},
$$

where a positive (negative) value of $\alpha$ means that the electric current flows parallel (anti-parallel) to the magnetic field. 
While a low plasma- $\beta$ is a reasonable justification for using force-free models, the opposite is not true. A high (of order one or more) plasma- $\beta$ does not exclude force-free magnetic fields. If the non-magnetic forces compensate each other (e.g., the plasma pressure gradient is compensated by the gravity force in a magneto-static equilibrium) then the Lorentz force can still vanish, even if $\beta$ is not small. In the general high- $\beta$ case, however, non-magnetic forces have to be considered self-consistently, e.g., in a magneto-static or stationary MHD model. We will only summarize some basics about the possibilities and problems of force-free models and avoid mathematical and computational details. For a more detailed overview on the methods used to compute solar force-free fields, see Wiegelmann and Sakurai (2012). Depending on the forcefree parameter (or function), $\alpha$, one distinguishes between potential (current-free) fields $(\alpha=0)$, linear force-free fields (LFF; $\alpha$ is globally constant) and the general case that $\alpha$ changes in space, i.e., the non-linear force-free (NLFF) approach.

\subsubsection{Potential and linear force-free fields}

The simplest case, a potential field, requires only the LOS photospheric magnetic field component as boundary condition. Current-free equilibria are mathematically simple and represent the lowest possible energy state of a coronal magnetic field. For computations on a global scale (PFSS models), one assumes that all field lines become radial at the ("source surface" at about 2.5 solar radii; see Schatten et al. 1969, for details). Potential field models are popular because they are easy to compute and are capable of reproducing the basic coronal magnetic field structure. More sophisticated methods (as discussed in the following) are numerically expensive and often use a current-free field solution as initial guess for an iteratively sought, non-potential solution.

To employ a LFF magnetic field model, only the photospheric LOS magnetic field component is required as well, but such models contain one additional free parameter $(\alpha)$. The value of $\alpha$ (constant in space) can be inferred from additional observations, e.g., in the form of an average value of the entire photospheric distribution of $\alpha=(\nabla \times$ B $)_{z} / B_{z}$. Note that $\alpha$ is the ratio of the vertical (LOS) current density and the vertical (LOS) magnetic field magnitude, and that the vertical (LOS) current density can be derived from the horizontal (transverse) magnetic field. (In that case, the knowledge of all three vector components of the magnetic field is required.) Alternatively, $\alpha$ can be deduced and/or optimized by the comparison of model magnetic field lines and coronal observations (either directly with coronal loops seen in EUV images, or coronal loops extracted from such images; and see also Sect. 2.4.1).

On global scales, LFF models are mathematically and computationally possible, but are not frequently employed, mainly for two reasons. Firstly, the maximum allowed value of $\alpha$ scales with the inverse of the length scale of the computational domain. Consequently very small values of $\alpha$ are possible but they are so small that they have no significant effect (i.e., the resulting magnetic field is almost similar to a potential field configurations). Secondly, observations show that both signs of $\alpha$ can be present in different regions on the Sun, at the same time. This is a contradiction to the LFF assumption, namely that $\alpha$ is constant (i.e., has the same value for different regions on the Sun). 
On smaller scales (in particular to analyze ARs), however, LFF models were used, though more frequently before the time when vector magnetograms started to became routinely available (as provided to date by, e.g., SDO/HMI). On these smaller scales, the maximum value of $\alpha$ can be significantly larger than on global scales and consequently active-region LFF fields can be very different from potential ones, e.g., the associated field lines can be sheared. Also for LFF models employed on active-region scales, however, the observation of different values of $\alpha$ in different portions of the same AR contradicts the basic assumption of a single value of $\alpha$ being representative for the entire AR under consideration.

\subsubsection{Non-linear force-free fields}

Given the limitations of potential and LFF approaches (as discussed above) for a meaningful and self-consistent modeling of coronal magnetic fields, one has to take into account that $\alpha$ is a function of position. This spatial dependence is accounted for in NLFF models, which are much more challenging, both mathematically (one has to solve non-linear equations) and observationally (mostly photospheric vector magnetograms are required as input, instead of just the longitudinal (vertical) field component). Measurement inaccuracies in photospheric vector magnetograms (e.g., due to noisy Stokes profiles and instrumental effects) affect the quality of NLFF coronal magnetic field models. The modeled coronal field, however, is less sensitive to these measurement errors than the photospheric field vector itself (Wiegelmann et al. 2010b). A review on methods for computing NLFF fields has been given by Wiegelmann (2008). The corresponding numerical implementations have been intensively reviewed, and repeatedly evaluated and improved within the last decade (see Schrijver et al. 2006; Metcalf et al. 2008; Schrijver et al. 2008; De Rosa et al. 2009). The numerical schemes have been implemented in cartesian and spherical geometry to perform active region and global magnetic field modeling, respectively. As boundary condition, either the magnetic field vector at the bottom boundary of the computational domain or, alternatively, the vertical magnetic field and vertical electric current density is usually required.

A difficulty arises from the fact that the plasma in the corona is a low- $\beta$ plasma, but that of the photosphere is not. In the photosphere, $\beta$ is on average of the order of unity or more (Gary 2001), although locally considerable smaller values may be found (e.g., in the interiors of magnetic elements; see Zayer et al. 1990; Rüedi et al. 1992). Note that a non-vanishing plasma- $\beta$ does not exclude the existence of a force-free field, but one has to be careful when using photospheric measurements as boundary condition for NLFF computations. Because then it cannot be guaranteed that the photospheric magnetic field vector is consistent with the assumption of a force-free field in the corona. One can find out whether the vector magnetic field measurements are consistent by writing the force-free equations as the divergence of the Maxwell stress tensor, integration over the entire computational volume and applying Gauss' law. For force-free consistency, the value of the resulting surface integrals has to vanish (see Aly 1989, for details), or in practice must then be sufficiently small. Theoretically, the surface integrals need to be evaluated over the entire boundary of the computational domain, but in practice this is only possible for the bottom (photospheric) boundary, 
where the field is measured. This is justified for ARs that are surrounded by weak (quiet Sun) fields where the gross part of the magnetic flux closes within the AR (i.e., on the bottom boundary) and the contribution of the other boundaries can be neglected.

Only exceptionally, however, active-region vector magnetograms fulfill the forcefree criteria (for such an example see Wiegelmann et al. 2012). In the majority of cases, they are not force-free, simply because the photosphere is a non-force-free environment. Additionally, polarization signals are often affected by the temperature in the sampled magnetic features and introduce biases between, e.g., sunspots and magnetic elements forming plage regions (Grossmann-Doerth et al. 1987; Solanki 1993). To circumvent this problem, a procedure-dubbed "preprocessing" has been developed. The method uses (force-free inconsistent) photospheric vector field measurements as input and provides a force-free consistent vector field as output (see Wiegelmann et al. 2006, for details). An alternative is to measure the magnetic field vector higher in the solar atmosphere, e.g., in the low- $\beta$ chromosphere (exclusively, or in addition to photospheric measurements).

To our knowledge, the first and so far only NLFF extrapolation from vector magnetograms observed simultaneously at multiple heights (at a photospheric and chromospheric level) has been performed by Yelles Chaouche et al. (2012), to study the structure of an AR filament. One difficulty in combining and comparing two such data sets is that the exact height in the atmosphere of the chromospheric measurement is unknown. As a reasonable approximation, the authors assumed that the chromospheric measurements refer to the height of best agreement with the NLFF reconstruction based on the photospheric vector field (about $2 \mathrm{Mm}$ above the solar surface).

Despite the difficulties discussed above, NLFF extrapolations are a powerful tool for deriving the 3D coronal magnetic field above ARs. On the other hand, the applicability of force-free models to quiet-Sun magnetic fields is questionable because it is very likely neither force-free nor quasi-steady (see Schrijver and van Ballegooijen 2005, and Sect. 5.2.5 for details).

\subsection{MHD models}

\subsubsection{MHD models of the coronal magnetic field}

A full understanding of the physical processes in the upper solar atmosphere requires the knowledge of the plasma that populates the investigated magnetic structures. Deriving these properties in the outer solar atmosphere, however, remains a challenging task. Most commonly used models for a self-consistent description of the plasma and magnetic field are based on the MHD approximation. Interestingly, even though the MHD approximation is strictly valid only in collisional plasmas, the collision-free coronal plasma is often modeled using such an approach. More sophisticated, collisionless kinetic models cannot be applied to model large-scale structures in the solar corona since the considered scales are several orders of magnitude larger than the relevant (microscopic) scales which have to be resolved in kinetic simulations (e.g., the gyroradius or Debye-length). This approach, however, is frequently applied to model the solar wind plasma (see review by Marsch 2006). 
One approach to derive plasma quantities, which can then be compared to observations, is forward modeling aided by time-dependent MHD simulations (see Peter et al. 2006). As an initial state, a potential field is computed from the measured photospheric (LOS or vertical) magnetic field component. (Note that for MHD simulations, the magnetic field data have usually to be scaled to a lower spatial resolution.) A strength of the forward MHD modeling technique is that the resulting plasma quantities can be used to compute synthetic spectra, which can be compared with observed chromospheric and coronal images/spectra (e.g.Peter et al. 2006, using SOHO/SUMER EUV data).

\subsubsection{MHS models}

A simpler approach, when refraining from performing numerically expensive timedependent MHD simulations is to use a reduced set of equations, e.g., MHS or stationary MHD. This allows a self-consistent modeling of magnetic field and plasma e.g., in the high- $\beta$ regimes containing the photosphere and lower chromosphere, and beyond the source surface in global simulations. Generally, these equilibria require the computation of non-linear equations, which are numerically even more challenging (and slower converging) than the set of NLFF equations, in particular, in a mixed- $\beta$ plasma (see Wiegelmann and Neukirch 2006; Wiegelmann et al. 2007, for an implementation in cartesian and spherical geometry, respectively).

Mathematically simpler, and computationally much faster, is the subclass of MS models, which are based on the assumption that electric currents flow on spherical shells perpendicular to gravity (resulting in horizontal,i.e., parallel to the lower boundary, currents in cartesian geometry). This approach allows linearizing the MS equations and solving them with a separation ansatz (see Low 1991; Bogdan and Low 1986; Neukirch 1995, for one cartesian and two spherical approaches, respectively). Because of the linearity of the underlying equations, a field-parallel electric current can be superposed (for a constant value of $\alpha$ ). The final current distribution consists of two parts: a LFF one and another one that compensates non-magnetic forces such as pressure gradients and gravity. These classes of MS equilibria require only LOS photospheric magnetograms as boundary conditions, which are relatively easy to implement and allow the specification of two free parameters (the force-free parameter $\alpha$ and additionally a parameter which controls the non-magnetic forces). The limitations on $\alpha$ are similar to those discussed for LFF modeling approaches (see Sect. 2.2). In these models, plasma pressure and density are computed self-consistently to compensate the Lorentz-force. Above a certain height the corresponding configurations become almost force-free, which in principle allow it to model a forced photosphere and chromosphere, together with a force-free corona above. A limitation of MS equilibria is that the two free parameters are globally constant and the method does not guarantee a positive plasma pressure and density. To ensure positive values of these quantities, one either has to add a sufficiently large background atmosphere (which may lead to unrealistically high values of the plasma- $\beta$ ), or is limited to small values of the parameter controlling the non-magnetic forces. Note that, as force-free approaches, MS models are only snapshots of the coronal field and the temporal evolution of such configurations can only occur as a series of equilibria, in response to temporally changing boundary conditions. 


\subsubsection{Flux transport models}

So far (for the aim of coronal magnetic field modeling), we have discussed only the coronal response to photospheric changes, but did not try to understand the evolution of the photospheric field itself. This can be done on a large (global) scale with the help of flux transport models (Leighton 1964, and for recent reviews see chapter 2 in Mackay and Yeates 2012 as well as Jiang et al. 2014). The aim of magnetic flux transport models is to simulate how (newly emerged) flux is transported horizontally on the solar surface, i.e., in the photosphere. The magnetic field is assumed to be radially oriented. The main contributing flows and velocities on large scales are differential rotation and meridional flows. On smaller scales, convective processes on granular and supergranular scales become important too, where the granular scales are generally ignored.

A natural application of flux transport models is to compute the evolution of active region or global coronal fields (see Sheeley et al. 1987; Baumann et al. 2004), as well as to investigate the development, structure and decay of polar CHs (see Sheeley et al. 1989), and to estimate the Sun's open magnetic flux. Flux transport computations performed in recent times often start from observed magnetograms, e.g., full disk (Schrijver and De Rosa 2003) or synoptic (Durrant and McCloughan 2004) LOS magnetograms from $\mathrm{SOHO/MDI}$. As a welcome side product, fluxes are obtained also for regions where no LOS measurements can be performed or where they are not reliable (i.e., at the far side and around the poles of the Sun, respectively). Additionally, such computations can be used to compensate gaps in the original full-disk or synoptic LOS data. To our knowledge, current flux transport models provide only the radial component of the photospheric field (i.e., not the full field vector), however.

For the aim of coronal magnetic field modeling, the resulting (synthetic) magnetic flux maps can be used in a similar fashion as LOS magnetogram data. Most popular for combined models of photospheric flux transport and coronal field models are global potential field models. A more sophisticated approach is to combine the flux transport model with a NLFF approach, based on a magneto-frictional MHD relaxation code (see van Ballegooijen et al. 2000; Mackay and van Ballegooijen 2006; Mackay and Yeates 2012). In contrast to the NLFF extrapolation technique based on vector magnetograms, this evolutionary method requires only the radial photospheric field component. Both, the photospheric and coronal magnetic field is evolved in time by a combined approach: the photospheric field by the flux transport model and the coronal field by the magneto-frictional code.

\subsection{Coronal stereoscopy, tomography and seismology}

Rather than measuring or modeling the magnetic field itself, we can get insights into the structure and shape (but not the field strength) of magnetic field lines by analyzing images of the emitting coronal plasma. This is possible because of, owing to the high conductivity, the coronal plasma is frozen into the field and thus serves as tracer of it. Special techniques (coronal stereoscopy and tomography) have been developed to reconstruct the 3D coronal structure from sets of simultaneously observed 2D images (see Aschwanden 2011, for a recent review). Here we briefly summarize the techniques relevant to magnetic field structures. 


\subsubsection{Stereoscopy and magnetic stereoscopy}

Stereoscopy is classically carried out with two (or more) images obtained from different vantage points. It is preferably done with clear solid edges, which are, however, not available for optically thin coronal structures (such as loops or plumes). While some early work on solar stereoscopy has been done from a single viewing direction (and using the rotation of the Sun to mimic multiple vantage points; see Berton and Sakurai 1985, for details), the application of both techniques got a big boost with the launch of the STEREO spacecrafts.

A natural approach is to compare and combine the results of coronal stereoscopy and magnetic field extrapolations from the photosphere (called magnetic stereoscopy; Wiegelmann and Inhester 2006 and for a review see Wiegelmann et al. 2009). In early applications, before vector magnetograms from SDO/HMI became routinely available, magnetic stereoscopy has been mainly performed with the help of LFF fields (based on LOS magnetograms). The method was designed to automatically find the optimal force-free parameter $\alpha$ of the LFF model (see Feng et al. 2007, for the first application of this method to STEREO/SECCHI images and SOHO/MDI magnetograms). Stereoscopy and magnetic field extrapolations have complementary strengths and weaknesses and it is by no means clear whether the reconstructed 3D loops validly represent the actual coronal loops (see De Rosa et al. 2009, for a comparison of force-free field modeling and stereoscopy). Nevertheless, a comparison of the result of both approaches at least serves as a consistency check and allows to approximate related uncertainties. Recently, some attempts have been made to use coronal information (either stereoscopically reconstructed 3D loops or 2D projections of loops extracted from coronal images) to constrain NLFF fields in addition to photospheric measurements (Malanushenko et al. 2014; Aschwanden et al. 2014; Chifu et al.2014, Astron. Astrophys., submitted).

Maps of optically thin radio emission (see Sect. 2.1.3.) can be treated basically similarly to EUV and SXR images. This is different for observations of optically thick sources, which have a similar opacity as a solid 3D body. Consequently for a given 3D magnetic field structure, one finds different (see section 3.5 in Aschwanden 2011, for details) gyroresonance layers that are visible as equi-contours in 2D images, dependent on frequency and harmonic. For slowly evolving magnetic fields, which remain almost static for a few days, the solar rotation can be used for a stereoscopic 3D reconstruction of the magnetic field structure. Here, the structures have a high opacity, making stereoscopy more straightforward compared to using images of optically thin sources. A comparison of this method with force-free magnetic field reconstruction methods based on photospheric data revealed that a potential field model failed to reconstruct a corresponding structure, whereas a NLFF approach showed a reasonable agreement (see Lee et al. 1999, for details).

\subsubsection{Tomography and vector tomography}

A complementary approach, which is specifically tuned to optically thin structures, is solar tomography. To our knowledge, this was first proposed by Davila (1994). This method uses LOS-integrated coronal images, preferably from multiple viewpoints, as 
input. Unfortunately, a large number of viewpoints are not available for solar observations and we are currently limited to a maximum of viewpoints (STEREO-A and B, plus either $S D O$ or $S O H O$ ). In future, Solar Orbiter will provide a fourth viewpoint. In principle, one can extend the number of viewpoints by taking images sequentially one after the other, while the Sun rotates. Because the vector tomographic inversion requires data from multiple viewpoints, one would need to observe the rotating Sun for several days if only one viewpoint, e.g. from Earth, is available. Then, the analysis is limited to static or slowly evolving structures.

Fortunately for the aim of coronal magnetic field investigation, the large-scale magnetic field structure changes more slowly compared with the plasma (which exhibits flows and reacts to, e.g., heating and cooling). Sources for a tomographic inversion are EUV images, white light images in which the radiation is dominated by Thompson scattering, and radio maps. Consequently, the physical conditions for both stereoscopy and tomography of the Sun are not ideal as compared with the stereoscopy of solid objects on Earth and one has to find suitable ways of combining different techniques to obtain the best scientific insight from available observations.

The inversion of magnetic field-related polarization signals is more challenging than inferring the plasma density, because the magnetic field is a vector. The corresponding vector tomography methods (Kramar et al. 2006, 2013) require, as in ordinary scalar tomography, an assumption for a regularization (in addition to LOS-integrated magnetic field measurements of magnetically sensitive coronal lines). This may be, e.g., the assumption of a solenoidal magnetic field. The corresponding regularization integrals require a boundary condition, which can be derived from photospheric magnetic field measurements, be they LOS or radial components. As a consequence, the vector tomographic inversion is not independent from photospheric measurements. In principle, there is a large potential of combining methods of NLFF coronal magnetic field models with vector tomographic inversions. Models for both approaches can be derived from optimization principles, which make a combination mathematically straightforward. The computational implementation, however, remains challenging.

\subsubsection{Coronal seismology}

While the stereoscopic reconstruction from coronal images provides only the 3D shape of coronal loops, but not their field strength, we can get insights into the coronal magnetic field strength by analyzing loop oscillations, which are often visible in time series of coronal images or spectra. The principle has been well known for several decades: the properties of waves traveling through a magnetized medium react to the magnetic field strength (for global seismology see Uchida 1970, and for local coronal seismology see Roberts et al. 1984). It is outside the scope of our article to review the rich history of coronal seismology, but the reader can find an overview of solar coronal waves and oscillations, including an introduction to coronal seismology in Nakariakov and Verwichte (2005). The basics for seismology are the analysis of waves, here within the limit of an MHD approach. MHD waves (slow and fast magneto-acoustic waves, and Alfvén waves) are sensitive to the magnetic field of the medium through which they travel. In principle, the method has similarities with using acoustic waves for helioseismology of the Sun's interior. Coronal images and spectra of high spatial resolution 
and time cadence (for example from TRACE, SDO/AIA and SOHO/SUMER, Hinode/EIS, respectively), allow a reliable application of coronal seismology to compute the magnetic field strength (see Van Doorsselaere et al. 2008).

\section{Global coronal magnetic fields}

\subsection{Magnetic field topology}

The structure of the solar corona is dominated by magnetic fields that emerge from below the solar surface and expand into the atmosphere. The plasma confined in these fields is visible in coronal images, and outlines open and closed coronal magnetic fields. Most of today's knowledge on the nature of the coronal magnetic field, however, was gained from coronal magnetic field models (see Sect. 2) and their comparison with observations of the radiation emitted by coronal plasma. Such a combination of modeling and observations is necessary due to the lack of routine direct measurements of the 3D magnetic field vector in the upper solar atmosphere (see review by Cargill 2009).

\subsubsection{Performance of PFSS models}

Platten et al. (2014) recently presented a detailed topological picture of the global solar corona based on PFSS modeling (see Sect. 2.2). Even during times of minimal solar activity, a quite complex picture of the coronal magnetic field is revealed (see Fig. 8). These authors summarized the building blocks of the coronal topology to include the neutral line at the source surface, separatrix surfaces which physically separate closed and open fields ("separatrix curtains"), and various types of smaller separatrix surfaces closing below the source surface. The coronal neutral line separates the large-scale opposite polarity regimes of the coronal magnetic field. Above the source surface (a regime not modeled within the PFSS approach) the neutral line is used as a proxy for the base of the heliospheric current sheet (for details on topological considerations of solar magnetic fields and the associated terminology see Longcope 2005, and see Sect. 4.2 for its representation on active-region scales).

PFSS models are widely used to picture the structure of the Sun's global magnetic field, basically owing to their mathematical simplicity and because only LOS photospheric magnetograms are required as boundary condition. The ability of such models to adequately reflect some of the observed structures, however, seems to be limited or at least dependent on the case studied and/or the specific analysis that is carried out. Thus, Wang et al. (2007) used a PFSS model to show that the magnetic structure of pseudo-streamers, as seen in $\mathrm{SOHO/LASCO}$ white light images, is rooted between open fields emanating from photospheric regions of the same polarity. Using the same model approach, Zhukov et al. (2008) investigated mid-latitude coronal streamers. During periods of high solar activity, however, they found no satisfactoring agreement of number and positions of the streamers, especially of those originating from polar regions. 


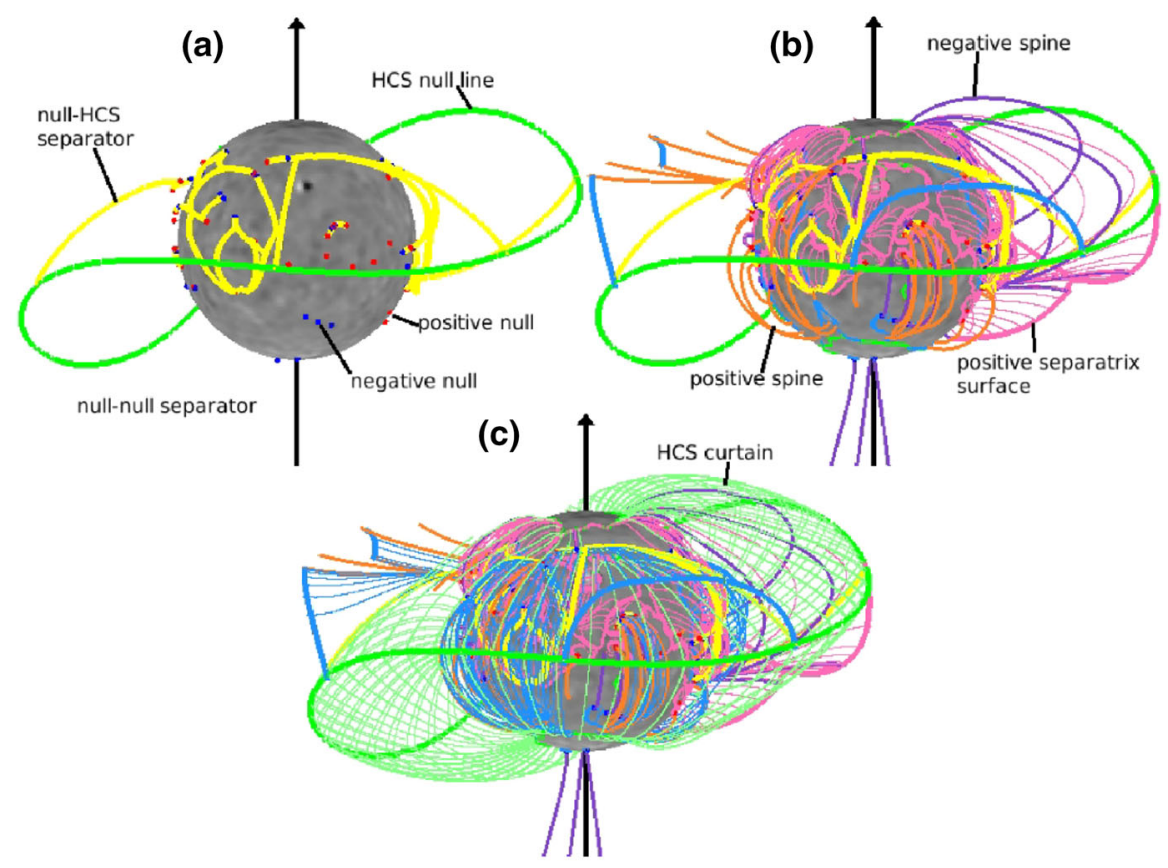

Fig. 8 Illustration of the basic elements of the global coronal magnetic-field topology, which is characteristic for the solar minimum period and was calculated from a PFSS model. The gray-scale sphere reflects the SOLIS/VSM radial component of the synoptic photospheric magnetic field. a The thick green line marks the neutral line on the source surface, which is the base of the heliospheric current sheet. Blue and red dots mark null points in the corona, i.e., places where the magnetic field vanishes. Yellow thick lines represent separators, i.e., field lines that mark the intersection of two separatrix surfaces from opposite polarity null points. b Separatrix surfaces (pink and blue lines) and spine field lines associated with the null points (orange and purple lines) are plotted on top of the features shown in a. c Superimposed on the features depicted in b is the "heliospheric current sheet curtain" (green thin lines) which separates closed from open fields. (Adapted from Figure 6 of Platten et al. 2014. Reproduced with permission from Astronomy and Astrophysics, (C) ESO)

One has to keep in mind that during times of high solar activity, large parts of the solar atmosphere are filled with non-potential magnetic fields, in particular, in ARs. A PFSS approach will then be limited in its success of reproducing the corresponding coronal magnetic field structure (Nitta and De Rosa 2008). Rust et al. (2008) estimated that, at best, in only about $50 \%$ of the cases, a PFSS model might be capable of reproducing the locations of open magnetic field structures associated to flaring ARs. More recently, Kramar et al. (2014) highlighted the limited success of PFSS models in reproducing coronal streamers even during periods of minimal solar activity. Depending on the height of the source surface, they found that the PFSS models could not or could only partly render the positions of coronal streamers and CHs seen in STEREO/EUVI $195 \AA$ A images.

One reason for the partial mismatch between PFSS model results and observed coronal structures is their current-free nature. A very likely other reason is the use of synoptic maps as photospheric boundary condition. Synoptic maps are usually created 
by combining the data near the central meridian of full disk, LOS magnetograms that are acquired daily over one Carrington rotation. They are thus representative for the activity near disk center in the course of one Carrington rotation, with the extreme longitudes on the map having been recorded 27 days apart. To model the locations of streamers and $\mathrm{CH}$ boundaries at a certain instance, however, real-time knowledge of the far-side magnetic field configuration needs to be available as well. Schrijver and De Rosa (2003) have shown that the lack of knowing the instantaneous magnetic field distribution on the far side of the Sun can be compensated. They used acoustic far-side imaging to forecast strong magnetic field concentrations before they appeared on the east solar limb. After including this information in the PFSS model, the $\mathrm{CH}$ boundaries as observed in SXR and He I synoptic maps matched quite well. This, and the above compilation of case studies involving PFSS modeling, implies that one needs to judge, by comparing with observations, the quality of PFSS model results in each case.

\subsubsection{Achievements of global MHD models}

In PFSS models, the effect of the solar wind is taken into account only by the assumption that all field lines become radial at the source surface. This is not sufficient to investigate wind properties themselves, because they require the application of models that include plasma flows, such as global MHD models. Such approaches are mathematically more complex and incorporate more physics than PFSS models. They consequently suffer from requiring also longer computation times (Riley et al. 2006; for a review see Mackay and Yeates 2012, see also Sect. 2.3).

If employed, they deliver properties beyond the coronal magnetic field, normally the density and temperature of the coronal structures. Riley et al. (2011) showed that such models have the ability to quantitatively reproduce the signatures seen in coronal images, provided additional assumptions on the coronal heating function are included (see Fig. 9). The north polar $\mathrm{CH}$ extends well down into the southern hemisphere and its shape is well recovered within the MHD model result. The location of the AR south of the solar equator is reproduced as well, but appears too bright compared to the observed EUV emission. Small-scale features as well as plasma emission from open field lines anchored in polar regions have not been recovered. Advanced MHD techniques have been used to successfully model the variation of the solar wind speed Hu et al. 2008; Nakamizo et al. 2009; Yang et al. 2007. This includes the fast and slow solar wind, the sector structure of the interplanetary magnetic field as well as the shape and location of CHs (see Sect. 6).

\subsection{Cross-equatorial fields}

Global observations often show loop structures extending across the solar equator, thereby connecting the two hemispheres of the Sun ("trans-equatorial loops"; TELs). These are systems of magnetic field lines bridging the solar equator that connects active and/or quiet-Sun regions. The TELs become sheared above the solar surface due to their line-tied footpoints being subject to differential rotation (in the long term), or vortex motions and/or the rotation of sunspots (on shorter time scales). This may 

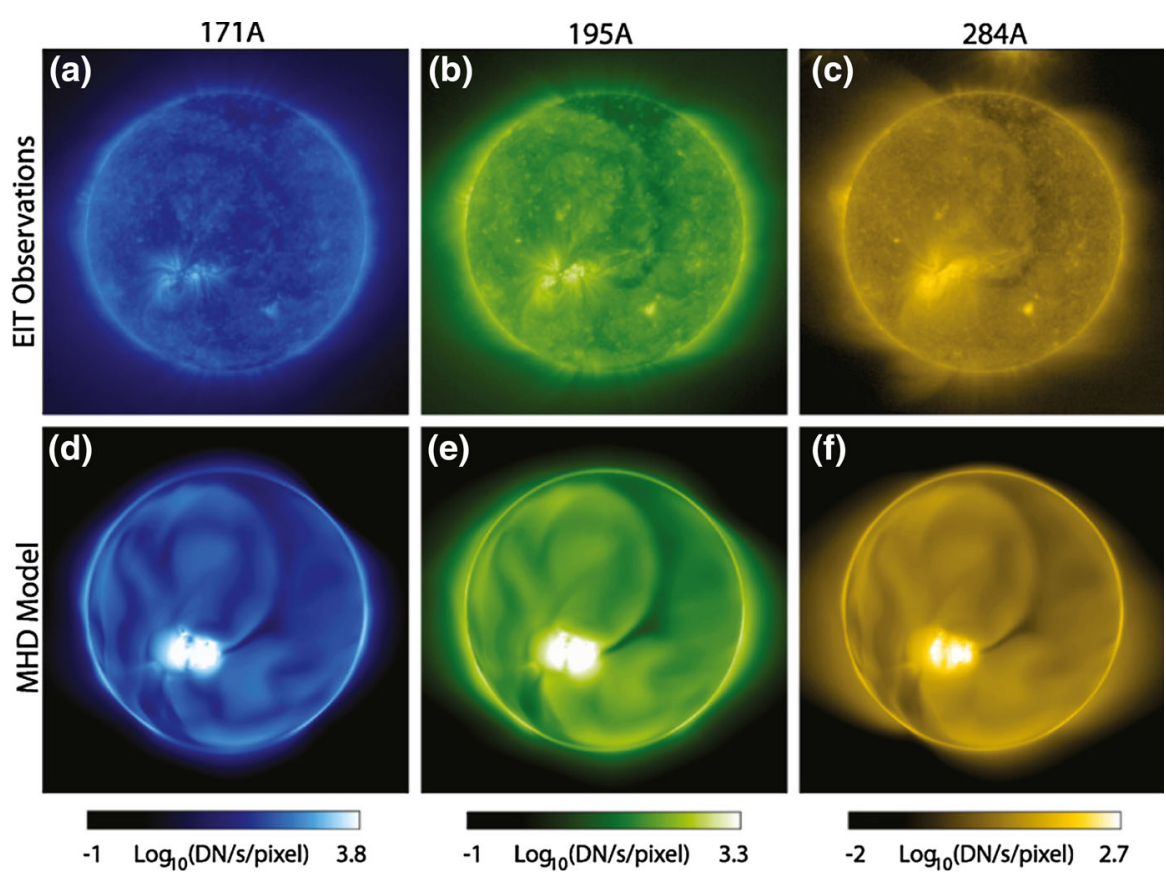

Fig. 9 Synoptic maps of the coronal EUV emission at a $171 \AA$, b $195 \AA$ and $\mathbf{c} 284 \AA$ for Carrington rotation 1913 (covering the period August 22 to September 18, 1996 at zero longitude), recorded by SOHO/EIT. A large $\mathrm{CH}$ (seen dark with respect to the quiet-Sun emission) extends from the north pole into the southern hemisphere. Synthetic d $171 \AA$, e $195 \AA$ and f $284 \AA$ spectroheliograms, computed from the densities and temperatures obtained with a global MHD model are shown below. Importantly, the overall brightness of the images compares. In addition, several features, including the cross-equatorial extension of the northern polar $\mathrm{CH}$ and the position of the AR south of the equator are well recovered. (Adapted from Figure 6 of Riley et al. 2011. With kind permission from Springer Science and Business Media)

also cause the constituent field lines of the TELs to become twisted around a common axis (Bao et al. 2002), which may then be observed in the form of a sigmoid in coronal images. Consequently, an observed sigmoidal structure does not necessarily imply that the underlying non-potential field geometry was already present when the fields emerged (Pevtsov et al. 1997).

\subsubsection{Creation of transequatorial loops}

The creation of TELs is still far from being understood, mainly because the most frequently employed 2D dynamo models cannot account for their intrinsic 3D nature and formation process (Jiang et al. 2007). 3D models aspiring to resolve the problem are still in their infancy (see Yeates and Muñoz-Jaramillo 2013; Miesch and Dikpati 2014, for recent applications).

Active-region magnetic fields that connect across the solar equator and form sigmoidal loop systems have been described by, Svestka et al. (1977) and Tsuneta (1996). They observed newly created loops, connecting across the Sun's equator in 
Yohkoh/SXT images and thus that the number of such newly brightened connections increased with time. Yokoyama and Masuda (2010) addressed the question how TELs, appearing bright in Yohkoh/SXT images, can connect very distant regions on the solar surface. In their picture, a series of reconnection processes between equator-bridging weak magnetic fields and their neighboring strong active-region fields reconfigures the magnetic connectivity such that strong equator-bridging fields develop. Besides, they pictured how this process may result in simultaneous chromospheric evaporation signatures at both footpoints of the TEL system (which had been reported observationally by Harra et al. 2003). Liu et al. (2010, 2011a) analyzed a coronal current sheet which appeared bright in SOHO/EIT $195 \AA$ images above a cusp-shaped flaring loop that connected locations on either side of the solar equator. They presented the first comprehensive set of observations, providing support for the standard picture of flare/CME events (usually referred to as the "CHSKP model" Carmichael 1964; Hirayama 1974; Sturrock 1996; Kopp and Pneuman 1976, and see also Sect. 4.3). These included the convergence in the legs of the TEL system in the area where the formation of a cusp-shaped flare loop was formed later on, co-temporal radio signatures, as well as an expanding post-flare arcade and coronal dimming in the atmosphere above it.

\subsubsection{Properties of transequatorial loops}

More frequently global NLFF modeling techniques are being used to investigate the magnetic connectivity between ARs located on either side of the solar equator (see Fig. 10). For instance, Tadesse et al. (2014) found that TELs carry only weak electric currents. The departure from a potential state, however, stresses the importance of using magnetic field models that allow departures from a current-free state. At the same time, global force-free magnetic field modeling can strongly depend on the boundary conditions supplied. Measurement errors are particularly high in the transverse component of weak quiet-Sun fields. Photospheric vector field measurements with different instrumentation can differ from each other and influence the corresponding coronal field models. Tadesse et al. (2013) performed a comparison of global NLFF models based on full-disk vector magnetograms from SOLIS/VSM and SDO/HMI. In this work, some of the TELs clearly observed in coronal images were reproduced with the help of NLFF modeling based on SOLIS/VSM data, but not from the modeling based on SDO/HMI data.

Pevtsov (2000) tested the importance of the chirality (handedness) of active-region magnetic fields for the formation of TEL systems. The results suggested that in roughly two-thirds of the cases the connected active-region fields were of the same handedness. Recently, Chen et al. (2010) examined the twist of a larger number of TELs (a subset of the samples analyzed by Chen et al. 2006). They found that the ones that linked ARs displayed an obvious sigmoidal shape and were related to a flaring activity stronger than C-class (i.e., peak SXR fluxes of $>10^{6} \mathrm{~W} \mathrm{~m}^{-2}$ ). They calculated the ratio $\tilde{L} / D$, where $\tilde{L}$ is the apparent length of the TEL system and $D$ is the apparent distance between the locations where the TEL system seems rooted at the solar surface. $\tilde{L}$ was measured by tracing the length of the coronal loops at the outer edges of the sigmoidal loop system, where they are well distinguished from the faint emission from the 

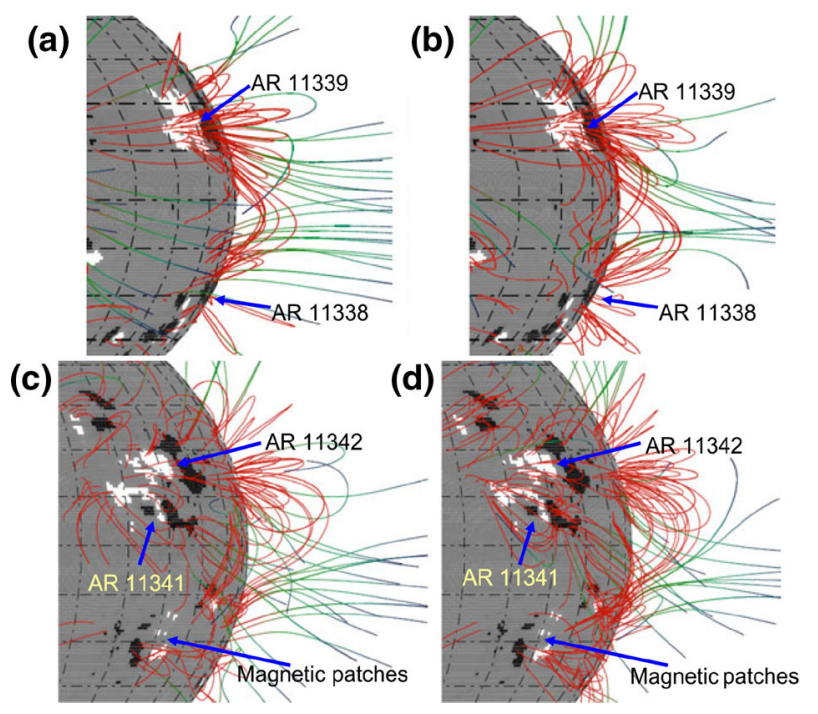

Fig. 10 Model magnetic field configurations connecting strong magnetic fields on either side of the solar equator. Selected field lines are calculated from a global a potential field and $\mathbf{b}$ a NLFF model connecting AR 11339 and AR 11338. Potential and NLFF model field lines connecting two ARs (11342 and 11341) to strong magnetic patches are shown in $\mathbf{c}$ and $\mathbf{d}$, respectively. Red and green line colors indicate closed and open magnetic field lines, respectively. The gray-scale background reflects the measured radial SDO/HMI magnetic field. It can be seen that the non-potential TELs deviate only little from a potential configuration. (Adapted from Figure 5 of Tadesse et al. 2014. With kind permission from Springer Science and Business Media)

(quiet Sun) background. Higher values of that ratio $\tilde{L} / D$ indicate a more pronounced sigmoidal shape and thus imply a stronger twisting of the associated field lines. They found that most of the TELs possess only weak sigmoidal shapes, indicating a low degree of non-potentiality. It appears that flares above C-class preferentially originate from structures of a specific amount of twist $(\tilde{L} / D \approx 1.4)$. It is an important future task to model the associated cross-equatorial $3 \mathrm{D}$ coronal magnetic field and its evolution with the help of global force-free and time-dependent MHD models to reveal the importance for eruptions to occur (see also Sect. 4.4).

\subsection{Spatio-temporal aspects of activity}

\subsubsection{Cyclic changes of the coronal magnetic field}

As the coronal field responds to photospheric changes, it also changes on global scales with an approximately decade-long periodicity. Bright loop systems appear at higher latitudes $\left(\gtrsim 30^{\circ}\right)$ at the beginning of a solar cycle and the activity belts progressively move closer to the equator as the cycle progresses.

Coronal holes vary in shape and position with the solar cycle as well (see review by Cranmer 2009, and Sect. 6). While polar CHs, if present at all, are found to cover only small areas around the poles during solar maximum, they tend to reach their largest 
extents around solar minimum and then occasionally extend to latitudes as low as $\approx 60^{\circ}$. Low-latitude $\mathrm{CHs}$ emerge preferentially around solar maximum and near the activity belts due to the occasional accumulation of unipolar flux during the emergence of ARs. If a sufficient amount of unipolar flux emerges and clusters together to form extended patches, the associated fields may be open. As a consequence, the open flux on global scales varies in the course of the solar cycle (its long-term variation was computed by Solanki et al. 2000, 2002; Schrijver et al. 2002; Jiang et al. 2011). Since the interplanetary magnetic field is fed by the magnetically open regions on the Sun, a similar modulation over the course of a solar cycle was expected too. Thus, it was surprising that the magnitude of the radial interplanetary magnetic field strength does not show a strong dependence on the activity level. Wang et al. (2000b) and Wang et al. (2000a) investigated why this is so and argued that the reduced area occupied by open fields around solar maximum is compensated by their, on average, higher field strengths. As a consequence, the open solar flux is nearly maintained throughout the solar cycle.

During solar activity maximum strongly emitting (bright) loop systems occupy a considerably larger volume within the corona. Associated active features, including flares (Bai 2003; Zhang et al. 2011) and coronal streamers (Li 2011) as well as superARs (i.e., ARs associated to repeated flaring and mass ejections, cf., Tian et al. 2002) were found to be distributed inhomogeneously in solar longitude. They seemingly relate to "active nests" or "active longitudes", which had originally been postulated based on similar trends seen in surface magnetic field observations. Chidambara Aiyar (1932), for instance, found sunspots to preferentially emerge at particular longitudes on the solar disk, which has been almost immediately attributed to projection effects and/or selection criteria by Carroll (1933). Follow-up studies concerning the preferred locations (longitudes) of sunspot formation delivered inconsistent and partly contradictory results. Even today, the possible number, migration, life times, longterm behavior and the particular method to track them remain a subject of debate (see Berdyugina and Usoskin 2003; Usoskin et al. 2005; Pelt et al. 2006; Usoskin et al. 2007; Weber et al. 2013; Gyenge et al. 2014).

\subsubsection{Association to dynamic events}

Mass ejecta cause severe changes of the coronal magnetic field, leaving it behind in a massively reconfigured configuration. Changes in the coronal magnetic field configuration, owing to magnetic reconnection of non-potential closed magnetic fields below coronal streamers, for example, are in fact thought to be the building blocks of the basic mechanism of coronal mass ejecta (for reviews see Forbes 2000; Chen 2011). Liu et al. (2009b), for instance, presented observations of short lived as well as of lasting deformations in association with an observed CME. The transient modifications were seen in the form of structures, neighboring the ejection site, pushed aside and bouncing back. Lasting distortions were observed in the form of displacements of the associated helmet streamer and the shrinkage of coronal holes (see Fig. 11).

On the other hand, it has also been reported that, in a considerable number of cases, the coronal environment barely responded to or appeared insensitive to the occurrence of mass ejections. This viewpoint has been championed by Sime (1989), who argued 


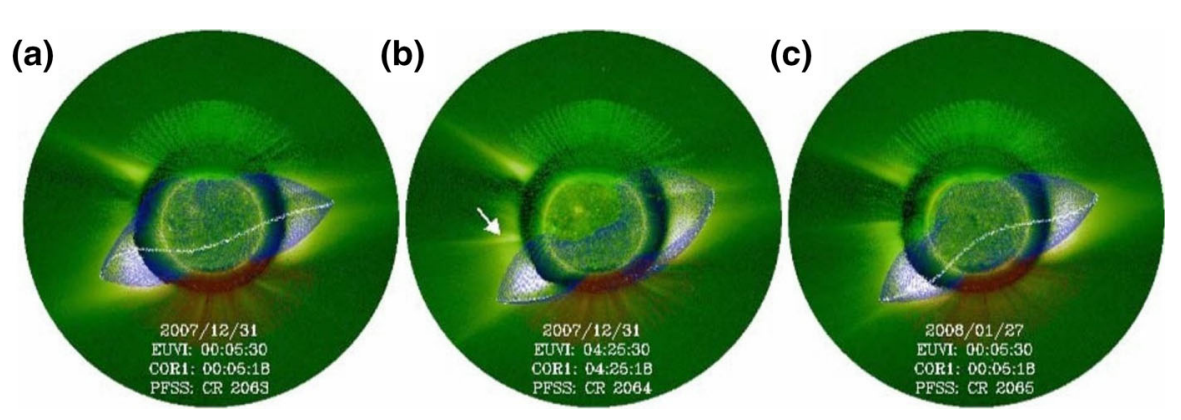

Fig. 11 Composite STEREO/SECCHI EUVI $195 \AA$ (Sun at the center of each image) and COR1 (surrounding disk) images a before, $\mathbf{b}$ immediately after and c 27 days after a CME. The white arrow in $\mathbf{b}$ indicates a streamer-like structure behind the CME which was interpreted as the current sheet in its wake, also because it did not exist before (compare a), nor about a month after the ejection (compare with c). The blue lines represent magnetic field lines calculated from a global PFSS model and projected onto the STEREO/SECCHI images. In a similar manner, the white line displays the source surface neutral line. It can be seen that, as a consequence of the CME, the coronal streamer migrated southwards (compare the position of the coronal streamer above the south-east limb of the Sun in $\mathbf{a}$ and $\mathbf{b}$ ) but survived and persisted for more than one additional solar rotation (note the location of the streamer in c). (Figure 3 of Liu et al. 2009b. (C) AAS. Reproduced with permission)

that the global evolution of the coronal magnetic field gives rise to CMEs but is not really influenced by them. In other words, the ejecta may be associated to the streamer belt but may not have a lasting effect on it. This was supported by the observation that the streamer belt appeared to reestablish itself within a few days after ejections (Zhao and Hoeksema 1996). Although, it is certain that CMEs are associated with the belt of coronal streamers (Hundhausen 1993), the disruption (or disappearance) of an associated streamer was observed in only $\approx 15 \%$ of the cases observed (Subramanian et al. 1999; Floyd et al. 2014). In summary, whether mass ejections are only a response to or a contributing factor to the coronal restructuring is not yet clear (Liu et al. 2009b).

It is generally agreed, however, that only mass ejections (which inevitably drag the embedded magnetic field along) are capable of physically reducing the coronal magnetic helicity, which is tightly related to the structural properties of the magnetic field (Moffatt 1969). We discuss this in the following.

\subsection{Magnetic helicity budget}

\subsubsection{Helicity dissipation and helicity transport}

Magnetic helicity is dissipated on significantly longer scales than the magnetic field and consequently the dissipation time in the corona is too long to relevantly reduce the helicity (Berger 1984). For the helicity budget of ARs and quiet-Sun regions see Sects. 4.6 and 5.2.6, respectively. Since magnetic helicity cannot be efficiently dissipated, it is approximately conserved in the absence of ejecta, i.e., the magnetic helicity in the solar corona will continuously buildup. This has important consequences for the magnetic field relaxation towards a lower energy state. It implies that in the course of a flare, but lacking a mass expulsion, a non-potential field can only relax 
to another, lower-energy configuration of the same helicity content (i.e., a constant- $\alpha$ field; see Taylor 1974; Heyvaerts and Priest 1984; Taylor 1986). However, the coronal magnetic field may never entirely relax to this constant- $\alpha$ (conserved-helicity) state, because the required "complete" reconnection is not expected to occur due to the linetying of the coronal magnetic field structures at photospheric levels. This also inhibits the simultaneous formation of numerous current sheets (see Antiochos and DeVore 1999, and references therein).

The majority of ejecta, including CMEs associated with disappearing filaments (Yurchyshyn et al. 2001; Cho et al. 2013) or eruptive X-ray loops (Mandrini et al. 2005; Zheng et al. 2011), propagate away from the Sun in the form of magnetic clouds (MCs) and hence actively carry helicity away. MCs are force-free regions of enhanced magnetic field strength, with the field vector monotonically rotating as they journey with the solar wind. There are the moving large-scale helical structures described by Burlaga et al. (1981), Burlaga (1988). They are a subset of interplanetary CMEs that consist of plasma and magnetic field, expanding behind a shock wave into interplanetary space. Gopalswamy et al. (1998) suggested that MCs originate from the structure overlying an eruptive prominence and its associated CME and include the coronal cavity and a bright frontal structure. A typical MC may carry a magnetic helicity of some $10^{41}$ to $10^{43} \mathrm{Mx}^{2}$ (Cho et al. 2013). This, and considerations based on surface magnetic field measurements, permit to estimate the amount of helicity transported away on a global scale during one solar cycle as $\approx 10^{45}$ to $10^{46} \mathrm{Mx}^{2}$ (Bieber and Rust 1995; Rust 1997; DeVore 2000; Berger and Ruzmaikin 2000; Georgoulis et al. 2009; Zhang and Yang 2013).

\subsubsection{Hemispheric trends}

When estimating the global helicity budget, it is of importance to take the Sun's differential rotation into account. DeVore (2000) estimated the effect which the Sun's differential rotation has on the shearing of an active-region magnetic field. He estimated an accumulated helicity as $\approx 10^{43} \mathrm{Mx}^{2}$ during a characteristic active-region lifetime of $\approx 120$ days. However, the differential rotation cannot represent the only source of helicity supply to the corona Démoulin et al. (2002). Georgoulis et al. (2009) suspected that the contribution of differential rotation to the total amount of injected magnetic helicity amounts only to about $20 \%$. The dominant source of injection, they argued, must be due to the plasma flows within ARs (see Sect. 4.6.1). This agrees with earlier results which stated that the amount of helicity injected by differential rotation in ARs may comprise roughly 10 to $50 \%$ of that injected by motions within the ARs themselves (Démoulin and Pariat 2009, and references therein).

The injection of magnetic helicity does not appear to display any periodicity, indicating it to be a rather unforeseeable process (Georgoulis et al. 2009). Although temporal periodic patterns have not been found, some systematics regarding the spatial distribution of helical features on a global scale are known. Several observational features indicating the handedness of structures, such as sunspot whorls (Hale 1925; Richardson 1941, and references therein), chirality of filaments (Rust 1967; Pevtsov and Balasubramaniam 2003; Bernasconi et al. 2005) and S-shaped coronal X-ray brightening (Rust and Kumar 1996; Canfield et al. 1999) revealed a dominant pos- 
itive helicity pattern in the northern solar hemisphere and a dominant negative one south of the solar equator. The associated magnetic fields in the northern and southern hemisphere are dominated by right- and left-handedness, respectively (Seehafer 1990; Pevtsov et al. 1995). Interestingly, these patterns do not change from one activity cycle to the next.

This tendency, however, was found to be less pronounced for active-region filaments than for filaments in the QS (Pevtsov and Balasubramaniam 2003). Bao et al. (2002) gave a hint why there is a weaker hemispheric trend of active-region magnetic fields. They argued that the chirality introduced by the same mechanism acting on a rising flux tube throughout the convection zone, or acting on the flux tube after emergence through the photosphere may be opposite. The dominant contribution would then determine the chirality of an AR which would not necessarily follow the hemispheric trend. Using a magneto-frictional model, Yeates et al. (2008) were able to reproduce the skew of more than $90 \%$ of considered filaments (observed in $\mathrm{H} \alpha$ images) correctly, even including exceptions from the hemispheric trend. Yeates and Mackay (2012) recently presented the first long-term simulation of the chirality of high-latitude filaments and were able to recover the hemispheric trend. According to them, the apparent handedness depends on which of the two effects, creation of helicity by differential rotation or its transport from active latitudes, is stronger. They found that the latter is generally stronger, except during the early years of a solar cycle. Further evidence for the dependence of the chirality of features in the solar atmosphere on the phase of the solar cycle comes from SXR loops (Zhang et al. 2010b) and active-region magnetic fields (Zhang et al. 2010a).

Having started on magnetic fields on active-region scales, we continue to discuss their magnetic and helicity properties in the next section.

\section{Coronal active-region magnetic fields}

\subsection{Coronal loops}

The frozen-in condition for the coronal plasma and magnetic field is valid in most of the coronal environment. The sole exceptions are the small diffusion regions in strong current concentrations, the so-called "current sheets" (see Sect. 4.2). Much of the plasma in the corona is confined by the magnetic field in the form of thin closed flux tubes. The loop plasma may be heated by a number of possible mechanisms (for a review see Reale 2010). As a consequence, pressure and density of the gas within the magnetic loops are enhanced compared to the values reached by the surrounding gas and the coronal loops appear bright in coronal radiation. This property is of great advantage for the investigation of the dynamics in the corona: the bright structures serve as an indirect tracer of the coronal magnetic field.

Owing to its high temperature the coronal plasma emits radiation predominantly at X-ray, EUV and radio wavelengths. The bulk of the coronal loops hosts plasma of temperatures of $\approx 10^{5} \mathrm{~K}$ to $\approx 10 \mathrm{MK}$. The lower and upper limits are representative of cool and flaring loops, respectively (Reale 2010). Coronal loops often fan out from relatively compact footpoints at the solar surface, arranging themselves in dome- 
like structures with multiple layers. The individual loops are thought to have a very narrow distribution of temperatures within the loops themselves-neglecting their footpoints - though the loops within an AR may have very different temperatures (Del Zanna and Mason 2003; Aschwanden and Boerner 2011). In the corona, where the field fans out with height, thin constituent strands are discernible. At present, several spectral EUV channels are used to observe and study coronal loop fine structures since they appear well defined around the wavelengths 171 and $193 \AA$. At the highest spatial resolution presently achievable, in the form of $\mathrm{Hi}-\mathrm{C}$ data, the density and temperature structure across the observed thin strands varies on a spatial scale of roughly $0 . ' 1$; alternatively, one may state that a diameter of $0 . ' 1$ represents an upper limit for the strands which make up an observed loop (Cirtain et al. 2013; Peter et al. 2013).

It has long been puzzling why especially the loops seen in coronal images do not show a significant variation of their width with height in the atmosphere. Given magnetic flux tubes expand with height in the solar atmosphere, one would naturally expect this to be reflected in form of a clear height dependence of the emission observed from the thin threads which compose the flux tubes (DeForest 2007). Instead, an apparently constant cross section and more or less constant brightness along the loops, but no significant expansion was observed at the two wavelengths mentioned above. This is the result of the analysis of coronal loops seen in Yohkoh/SXT images (Klimchuk et al. 1992), and EUV observations with TRACE (DeForest 2007) as well as with SDO/AIA (Aschwanden and Boerner 2011). It has been argued that this might just reflect the fact that the coronal loops are entities of a constant diameter (Klimchuk 2000), although force-free magnetic field models do not support such an interpretation.

Physical reasons for the geometric distribution of the observed coronal loop emission are now beginning to come from numerical experiments. Peter and Bingert (2012) used an MHD model to investigate the temporal evolution of the corresponding synthesized coronal emission. They were able to show how emission of seemingly constant width may arise from an expanding flux tube. They argued that the plasma indeed fills the fanning-out magnetic field structure, but that it does not equally contribute to the emission perpendicular to the plasma loops' axis. The radiation from the plasma at the outer edges, i.e., the "envelope" of the expanding magnetic field is emitted from plasma at a lower temperature. Consequently, this radiation is missed in images showing the bulk of the emission at, say, $171 \AA$ and resulting in coronal images showing loops of nearly constant cross section. Peter and Bingert (2012) also pointed out that the appearance of the coronal loops may even be caused by the specific perspective at which the coronal loops are seen (see also Mok et al. 2008). Similar results have been found recently by Malanushenko and Schrijver (2013), who investigated a large sample of flux tubes that were based on a potential field model. They were able to assign well-known observed features to both, projection effects as well as the deviation of the flux tubes' cross section from a circle along its length. These include the enhanced brightness of loops which seemingly do not expand much in the plane of sky, compared to neighboring loops which seem to do so, and the characteristic elongated bright emission where loops turn towards the LOS (see Fig. 12). 

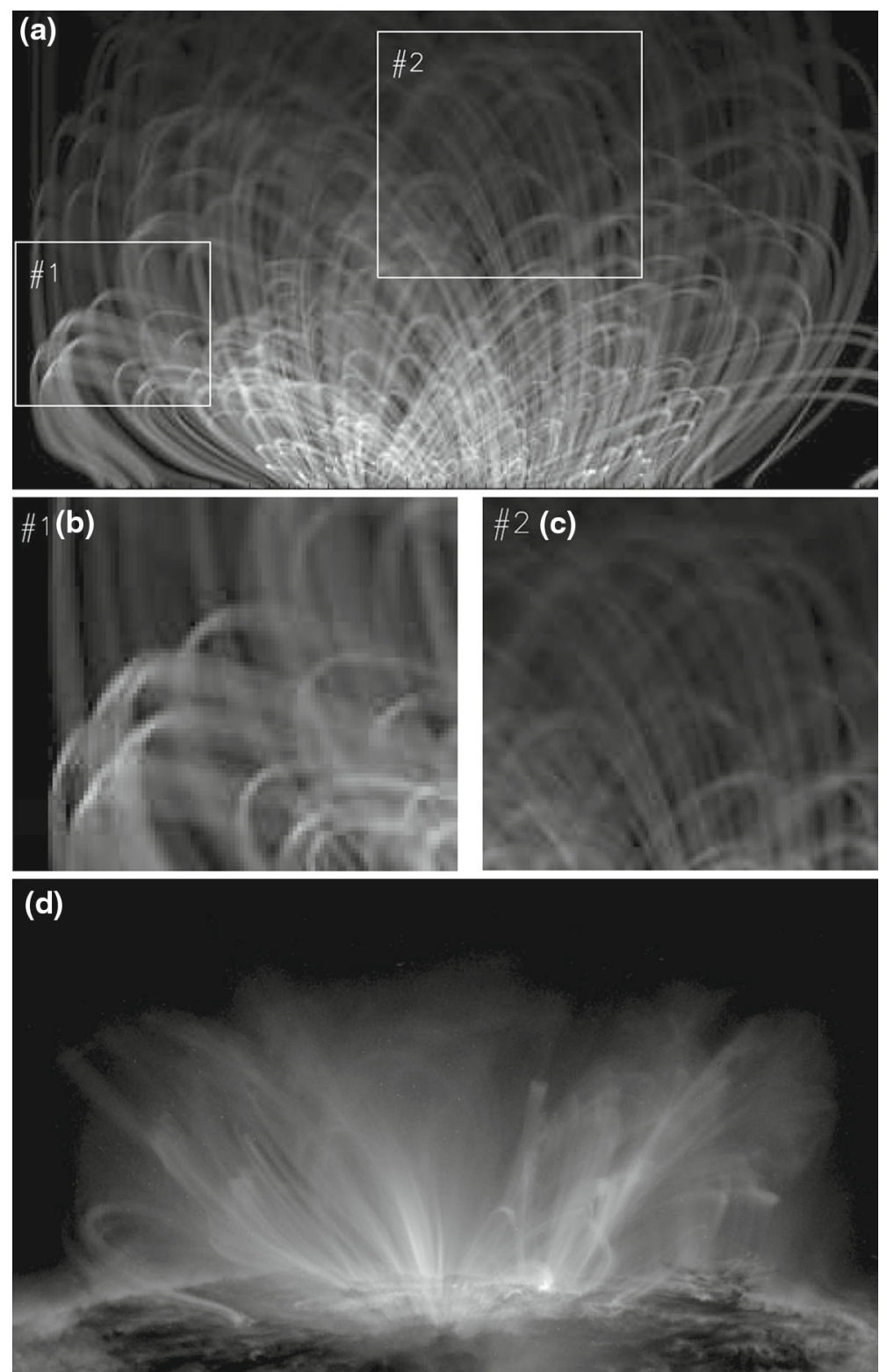

Fig. 12 a Coronal emission simulated from flux tubes (calculated from a potential magnetic field model) filled with hydrostatic, isothermal plasma. The gray scale reflects the squared column emission in arbitrary units. Rectangular areas labeled as \#1 and \#2 highlight areas where loops do not notably expand with respect to their neighbors and where loops turn towards the observer, respectively. Close-ups of these regions are shown in panels $\mathbf{b}$ and $\mathbf{c}$, respectively. d EUV emission from active-region loops as seen above the solar limb in an SDO/AIA $171 \AA$ image. The numerically synthesized brightness variations are well visible in the observed corona: the emission of loops with less varying apparent cross section is enhanced (compare panel b) and horizontally elongated where loops turn towards the LOS (compare panel c). (Adapted from Figures 17 and 18 of Malanushenko and Schrijver 2013. (C) AAS. Reproduced with permission) 


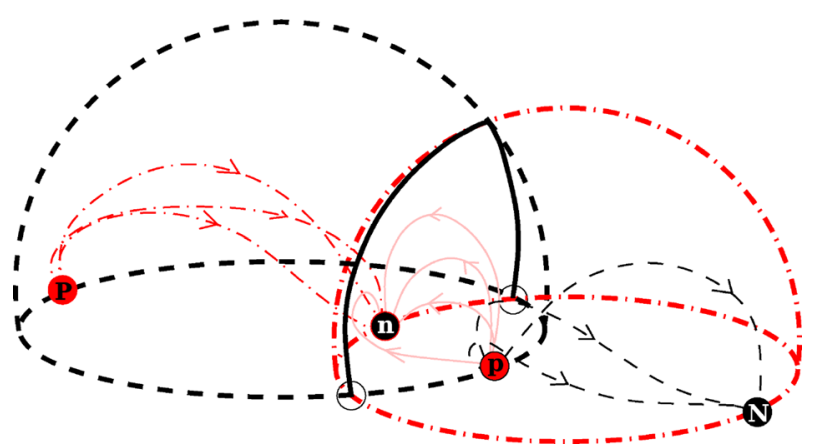

Fig. 13 Sketch of a 3D magnetic skeleton. Circles with red and black filling represent magnetic sources $(P, p)$ and sinks $(N, n)$, respectively, i.e., positive and negative flux concentrations. Black (dashed) and red (dash-dotted) curves represent sample field lines which close within the domain bounded by the positive and negative separatrix surfaces (thick red dash-dotted and thick black dashed outlined domes, respectively). The separator field line (black solid curve) runs where the positive and negative separatrix surfaces intersect, connecting two null points (black circles). It also marks the intersection of at least four distinct flux domains. Pink solid curves represent field lines that connect $p$ and $n$, which must close below the separator. Note that $P$ and $N$ can only be connected by field lines running outside of both separatrix surfaces, i.e., they have to bridge the separator. (Adapted from Priest and Forbes 2002)

\subsection{Local field topology}

The bright coronal loops within ARs connect photospheric locations of enhanced magnetic flux of opposite polarity. On a global scale, as discussed in Sect. 3, such loops are observed only within certain latitude bands, the activity belts. The activeregion fields are often composed of strong sunspot fields surrounded by plage regions of weaker field strengths. Prominent ensembles of active-region loops preferentially connect locations of strong magnetic flux inside an AR (i.e., sunspots and/or plage regions), or they have one footpoint located in an AR and the other in the enhanced flux of the surrounding network (Schrijver et al. 1999).

\subsubsection{Magnetic skeleton}

The building blocks of the coronal magnetic field include the locations of a vanishing magnetic field ("null points"), field lines which separate topologically distinct regions of space (forming "separatrix surfaces"), as well as special field lines at the intersection of such surfaces which connect two null points ("separators"). The sum of all these special locations, field lines and surfaces is called the "magnetic skeleton" (see Longcope 2005; Priest 2007, and Fig. 13). Separatrix surfaces and separators are places where the magnetic field is discontinuous, i.e., the field lines with footpoints on either side connect to rather different positions on the Sun. Consider, for instance, a field line which originates from the vicinity of the positive polarity $P$ (indicated by the red, dashed-dotted lines) in Fig. 13. If the positive polarity footpoint of the field line is located within the separatrix surface associated to $P$ (black dashed dome), the field line can only close down in the vicinity of the neighboring negative polarity $n$. If 
the footpoint, however, is shifted to a location just outside of the associated separatrix surface, the field line can no longer connect to $n$, because field lines do not cross separatrix surfaces or separators. Instead, it may connect to another neighboring negative polarity $N$ by running all along outside of the separatrix surfaces associated to $P$ and $N$ (red dash-dotted dome).

\subsubsection{Association to current-sheets and magnetic reconnection}

When a magnetic configuration evolves quasi-statically or dynamically, different flux domains develop as pictured above. It is possible to trace the different magnetic flux domains in the corona and their footprint on the solar surface, given that the 3D magnetic field configuration is known (Priest and Démoulin 1995; Démoulin et al. 1997; Démoulin 2006; Titov et al. 2002). One can determine the position of the conjugate polarity footpoint of a certain field line. Applying a horizontal shift to the location of the footpoint from which the field line calculation is started, the places of strongest variations regarding the location of the opposite polarity footpoint are detected, indicating the location of "quasi-separatrix layers" (QSLs; Priest and Démoulin 1995; Démoulin et al. 1997; Titov et al. 2002). There, the magnetic connectivity is not discontinuous but has steep gradients. This leads to the formation of current sheets (Titov 2007) in which electric currents may be efficiently dissipated (Aulanier et al. 2005; Bellan 2006; Pariat et al. 2009). Note that this implies that current sheets preferentially form where QSLs border each other (Schrijver et al. 2010).

Current sheets are regions in space where the magnetic field strength, and thus the magnetic energy density, is locally enhanced. In the collisionless environment of the solar corona, current sheets are transient features which diffuse away but, as long as they exist, they have important consequences for the field and plasma they contain. Most importantly, the resistivity within a current sheet is locally enhanced and as a consequence, unlike in the coronal surroundings of the current sheet, the plasma is - in contrary to the surrounding corona-not frozen into the field. Thus, they are favorable locations for changes of the magnetic topology to happen, i.e., where the magnetic field may change direction and/or magnitude (see chapter 2 in Priest (1982), Priest and Forbes (2007), chapter 6 in Priest (2014), and also Sect. 4.5.3).

As pointed out by Parnell et al. (2008), knowledge about the magnetic skeleton is necessary if one aims to determine the location, type, rate and frequency of reconnection events. Therefore, the magnetic skeleton has been investigated for complex magnetic field configurations also with the help of MHD experiments satisfying solarlike parameters. Maclean et al. (2009) performed MHD simulations, using a potential field based on $\mathrm{SOHO/MDI}$ observations as an initial equilibrium. They investigated the evolution of the magnetic field around an EUV bright point observed in TRACE $171 \AA$ images. They simulated the effect of the observed rotation of one of the main photospheric magnetic sources of a bright point. They could also show that the resulting buildup of electric currents may have enabled magnetic reconnection at the separatrix surfaces associated with the rotated magnetic source. They commented that more research must be undertaken to determine which parts of the separatrix surfaces host strong electric currents. This was partly addressed by Parnell et al. (2010), who studied the magnetic skeleton in the course of magnetic flux emergence into a pre-existing 
magnetic field. Their results indicated that locations along separators are favorable locations for magnetic reconnection to occur.

\subsubsection{Relation to dynamic phenomena}

Because the trigger for magnetic reconnection has often been suspected around an existing coronal null point or within a QSL, studies have been undertaken to relate flare-associated features to the magnetic topology of the corona (Luoni et al. 2007; Baker et al. 2009; van Driel-Gesztelyi et al. 2012; Sun et al. 2012a). Aulanier et al. (2000) inferred the location of a coronal null point and separatrix surfaces from a potential field reconstruction and highlighted that, a current-free model cannot be expected to reproduce the observations in detail—especially in the presence of strong shear. Nevertheless, it can be expected that it should be capable of recovering the basic, underlying coronal magnetic field topology. A similar finding was presented by Su et al. (2009), who employed, besides a potential field model, also a NLFF field model with an artificially inserted flux rope, forced to emerge into the force-free model corona. Their work revealed a line of coronal null points not only in the NLFF but also in the potential field representation. This supported the idea that a potential field model may indeed be sufficient to gain information on the basic coronal magnetic field topology. A less supportive conclusion was reached by Sun et al. (2012a), who found that the number and location of coronal null points were not the same for NLFF and current-free field models. Specifically, the position of a coronal null, as inferred from an NLFF field solution was clearly displaced from the location calculated from the associated potential field model. This mismatch, they argued, might have been caused by the high non-potentiality of the investigated active-region field which made the current-free model fail to realistically account for its structure.

Aulanier et al. (2000) and Su et al. (2009) found the shape of observed flare ribbons to be closely associated to the intersection of separatrix surfaces with the lower atmosphere. The spatial proximity between flare ribbons and QSLs agrees with the picture of confined flares (for reviews see Priest and Forbes 2002; Shibata and Magara 2011), which relates flare ribbon emissions to particles that are accelerated at the coronal reconnection site and that follow the separatrix field lines downward. Eventually encountering the denser layers of the lower atmosphere, they lose their energy due to collisions, but increase the radiation emitted at these locations by heating the surrounding plasma. Also using a potential field model, Masson et al. (2009) explained the flare ribbons observed in association with a small flare by the photospheric intersections of field lines that passed the vicinity of a coronal null point (similar to what was found by Chandra et al. 2009, see Fig. 14). They found that these field lines formed a surface which enclosed the flux domain of a "parasitic polarity" (that is a patch of a certain magnetic polarity surrounded by fields of opposite polarity) and that the geometry agreed closely with the theory of confined flares.

Note that magnetic field lines are fictitious constructs, meant to outline the direction of forcing by the magnetic field. Thus, they are often related to physical coronal structures, since charged particles in the corona feel the force exerted by the magnetic field (Lorentz force). As a consequence, for instance, heat conduction is most efficient along the magnetic field. Similarly, density enhancements and small localized distur- 

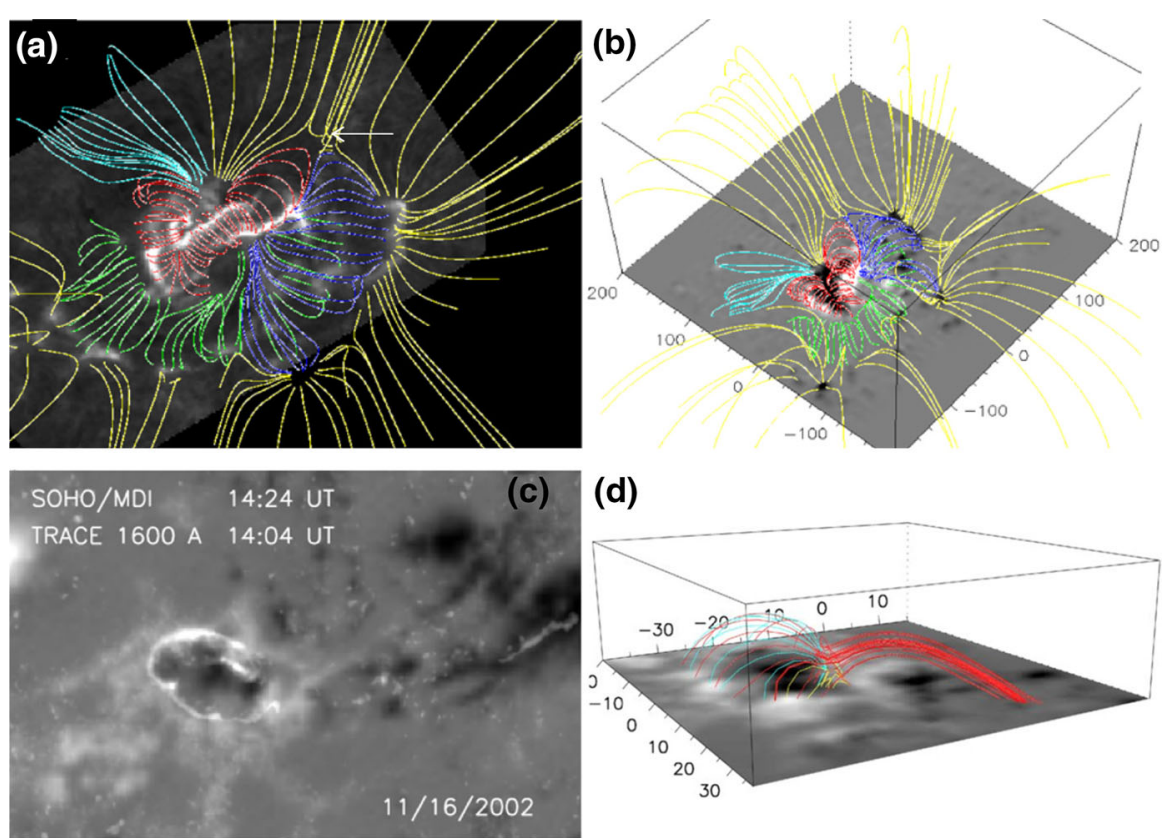

(d)

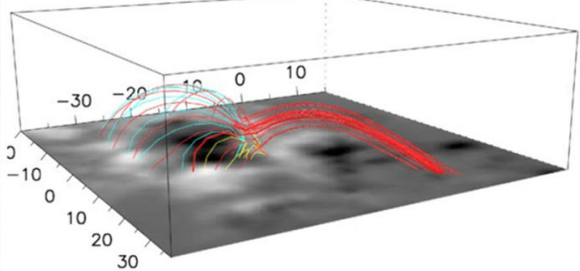

Fig. 14 a Magnetic topology of AR 10365 on 27 May 2003, estimated from a current-free field model. The white arrow in a indicates the possible location of a coronal null point, based on the associated current-free field configuration. Selected field lines that outline the basic magnetic field configuration are displayed on top of a near-in-time $\mathrm{H} \alpha$ image taken at the Solar Observatory Tower Meudon. Strongest emission in a outlines the location of flare ribbons (that is, where newly reconnected field lines are line-tied to the low atmosphere). b The same model configuration, shown on top of the SOHO/MDI LOS magnetogram (gray scale, where positive/negative polarity corresponds to white/black areas). Sample magnetic field lines, connecting the AR and its periphery are shown in yellow. Green, light and dark blue, as well as red field lines connect the observed flare ribbons to other regions within the AR. (Adapted from Figure 8 of Chandra et al. 2009. With kind permission from Springer Science and Business Media.) c TRACE 1,600 ̊̊ emission (bright ribbon and kernels) associated to a C-class flare on 11 November 2002, overlaid on a SOHO/MDI photospheric LOS magnetic field (gray-scale background; white/black represents positive/negative polarity) of AR 10191. d Sample field lines outlining the potential field reconstruction of the associated coronal field. The red, yellow and blue lines indicate a coronal null-point topology. (Adapted from Figures 2 and 3 of Masson et al. 2009. (C) AAS. Reproduced with permission)

bances propagate along field lines, and plasma flows are mechanically confined by the field (Longcope 2005). Such flows persist on time scales similar to that of the slow quasi-static evolution of the magnetic field, i.e., of the order of hours, and have indeed been found near QSLs. Early on, Marsch et al. (2004) were able to show the quasistatic nature of active-region flows and their close connection to the coronal magnetic field structure. Using a LFF field model, they were able to associate sharp changes in the Doppler velocity (inferred from SOHO/SUMER Dopplergrams) to the border between topologically different field configurations (open and closed). Furthermore, there seems to be a connection between the strength of the flows and the underlying magnetic field: the strongest flows seem to be associated to that portion of QSLs which are situated above strong magnetic fields (Baker et al. 2009). 


\subsection{Temporal evolution of active-region magnetic fields}

The active-region corona that spans from the coronal base until about $100 \mathrm{Mm}$ above the solar surface, is a low- $\beta$ environment (see Gary 2001, and Sect. 1.2.3). This implies that the evolution of the magnetic field dictates the mass, momentum and energy flow. This is because the dynamic pressure exerted by the solar wind within the Alfvén radius, i.e., the range within which any escaping material is forced to rotate rigidly with the Sun, is small compared to the magnetic pressure, the latter also well exceeding the gas pressure there (see chapter 9 in Stix 2002). The shuffling around the magnetic field lines at photospheric levels by the convective motions at (sub-)photospheric levels determines the temporal evolution of the coronal field above. Thanks to the high Alfvén velocity $\left(\approx 10^{3} \mathrm{~km} \mathrm{~s}^{-1}\right)$, the coronal plasma can quasi-statically adjust to the driving (sub-)surface convective motions (with characteristic speeds of a few $\mathrm{km} \mathrm{s}^{-1}$; see chapter 6 in Stix 2002). This, together with the underlying field topology determines the fate of coronal active-region magnetic fields. In most cases the evolution of the corona is a slow transition between neighboring equilibrium states.

A slow, quasi-static evolution means that the coronal magnetic fields adjust to the random or systematic motion of their line-tied photospheric footpoints without a sudden energy release, i.e., without a major eruption. Note that the condition of the magnetic field being frozen in the plasma and its more or less passive advection by photospheric flows is referred to as line-tying (see Longcope 2005). This does, however, not preclude the presence of dynamic phenomena. For instance, at the edges of ARs, outflows have often been observed with velocities of tens of $\mathrm{km} \mathrm{s}^{-1}$. This has actually been proposed to be important for the solar wind Brekke et al. (1997), Winebarger et al. (2001), Sakao et al. (2007). Such flows might arise from the reconnection between small-scale emerging fields and larger-scale open field lines of ARs (Liu and Su 2014). Also SXR jets are sometimes observed at the boundaries of ARs (Shimizu et al. 1994), often associated with parasitic polarities (Schmieder et al. 2013). But despite such small-scale events, for most of the time, the magnetic field of an AR evolves slowly and can be considered to be in equilibrium. At typical coronal conditions, the magnetic energy density — which is a measure for the magnitude of the associated pressure - is at least three orders of magnitude larger than the gravitational, thermal or kinetic energy density (Forbes 2000). Under equilibrium conditions, this implies that the Lorentz force vanishes and that the field can be considered to be forcefree (see Sect. 2.2). Once equilibrium is lost, however, this might no longer be true and dynamic forces come into play that compensate the non-vanishing magnetic forces.

\subsubsection{Dynamic evolution: eruptive phenomena}

Forced away from its equilibrium position, or, more precisely, from a quasi-static oscillation around its equilibrium position, a configuration of the coronal field may develop in two ways. Either it reaches a metastable state or a non-equilibrium state (Priest and Forbes 2002). A metastable configuration is stable with respect to small perturbations because it involves a stabilizing element. A corresponding coronal configuration would be in the form of, e.g., a twisted flux rope bridged by a loop arcade, where the latter holds the configuration steady (Sturrock et al. 2001). A non-equilibrium state, 
on the other hand, is unstable against any further perturbation and its evolution is then determined by the evolutionary path of that initially small perturbation. We are facing different types of evolution, in the form of a growth of the perturbation, an oscillation, or eventually a damping out. Given the appropriate trigger, a meta- or non-stable state may evolve further to a state where mass and/or energy is released due to an associated change of the field topology. Examples of force-free equilibria with stable and unstable configurations (dependent on model parameters) have been constructed by Titov and Démoulin (1999). Unstable configurations have been investigated with numerical MHD simulations by Török and Kliem (2005). Importantly, the morphology and dynamic evolution of a modeled erupting flux rope (an eruption triggered by an ideal kink instability) were found to be in agreement with an observed eruptive flare.

Associate releases of mass and/or energy are called flares, eruptive prominences and CMEs, depending on the emission and dynamics observed (for reviews see, Benz 2008; Fletcher et al. 2011; Mackay et al. 2010; Parenti 2014; Hudson et al. 2006; Webb and Howard 2012). Flares involve sudden enhancements of electromagnetic radiation, caused by accelerated particles and excessive heating of the coronal plasma. During an eruptive prominence, the material initially trapped inside is partially or completely expelled from the Sun. CMEs involve the expulsion of huge masses of coronal plasma into interplanetary space, spanning the range of roughly $\approx 10^{12} \mathrm{~kg}$ to $\approx 10^{16} \mathrm{~kg}$ (see Vourlidas et al. 2010, 2011, for a comprehensive analysis of CME masses during a full solar cycle). Many attempts have been made to develop models to describe the features associated with a loss of equilibrium, including the pre-eruptive coronal magnetic field structure, the triggering mechanism and the temporal evolution of the eruption itself. It is outside the scope of this review to summarize all existing models of eruptive processes in the solar atmosphere. Instead, we refer here to the comprehensive reviews on flare (Forbes et al. 2006; Chen 2011) and CME models (Forbes 2000; Shibata and Magara 2011).

\subsection{Favorable conditions for eruptions}

To find the conditions under which the magnetic field of a (part of an) ARs is likely to erupt, one has to analyze the corona as well as the underlying parts of the atmosphere before an eruption occurs (for reviews see Schrijver 2009; Chen 2011). Favorable conditions for eruptions include strongly twisted or sheared magnetic structures embedded in a less-sheared magnetic system (Hao et al. 2012), the rapid emergence/evolution of strong magnetic flux concentrations (Wang et al. 2004a; Sun et al. 2012b), and/or a complex magnetic field topology (Sun et al. 2012a).

\subsubsection{Magnetic flux emergence and complex magnetic field structure}

A complex magnetic field structure may include parasitic polarities, highly sheared fields, strong gradients and long PILs, not necessarily all at the same time or in the same event (van Driel-Gesztelyi and Culhane 2009). For magnetically complex ARs a tendency to be more CME productive has indeed been found (Chen et al. 2011). Large flares mainly originate from ARs with a complex photospheric magnetic field 
configuration, the largest ones from $\delta$-configurations (see review by Benz 2008, and references therein). Note that a $\delta$-configuration denotes umbrae of opposite polarity existing within a single penumbra. Only rarely are spotless regions reported to be the source of major flares (Ruzdjak et al. 1989; Sersen and Valnicek 1993; Li et al. 1995). M- and X-class flares are considered to be "major" flares in this context with SXR peak fluxes in the order of $10^{-5}$ and $10^{-4} \mathrm{~W} \mathrm{~m}^{-2}$, respectively. Ruzdjak et al. (1989), however, mentioned an enhanced occurrence rate of a specific type of eruption in spotless regions, namely two-ribbon flares, involving two progressively spreading bands of chromospheric emission.

As noted by Schrijver (2009), an eruption might not be triggered per se due to flux emergence but only if a sufficient amount of magnetic flux emerged. The imbalance of the emerging magnetic flux does not seem to be crucial for an eruption to occur, although it might play a role. Choudhary et al. (2002) noted that only roughly one-third of their analyzed cases showed a flux asymmetry of more than $10 \%$. A super-AR, however, was found to exhibit a flux imbalance of $\approx 40 \%$ which, they admitted, could have partly been caused by instrumental effects. It is worth noting that, such analyses often suffer the problem of projection effects which is noticeable in the degree of flux imbalance systematically increasing with distance from the disk center (Green et al. 2003). Chen and Wang (2012) recently revisited the importance of flux imbalance for the eruptive nature of ARs; they only considered ARs that fulfilled certain selection criteria, including being observed within $\pm 30^{\circ}$ from the central meridian and being associated to major flaring activity. They analyzed 14 ARs and found a significant imbalance of magnetic flux: more than half of the ARs showed a flux imbalance of $\gtrsim 20 \%$ (see also Tian et al. 2002; Romano and Zuccarello 2007). However, thermal effects on the photospheric line profiles can influence the determination of magnetic flux. This can lead to a seeming imbalance between the two polarities of an AR if, e.g., one polarity of the AR is composed more of sunspots, while the other polarity has more plage. Consequently, this topic needs further study.

\subsubsection{Magnetic shear and twist}

An eruption may also be triggered if, owing to the motion of its photospheric footpoints, a coronal loop arcade is sheared too much (Priest and Forbes 2002). It has long been known that the combination of strong fields and strong shear seem an essential ingredient to, for example, ribbon flares (Hagyard and Rabin 1986). Strong shear may develop due to sunspot motions, converging giant convection cells, as well as due to the emergence, cancelation and submergence of magnetic flux (see Hagyard et al. 1984a, and references therein; see also Wang 1994). Note, however, that it might not always be possible to judge whether horizontal flows caused the observed shear or if an arcade already emerged as a sheared configuration (Wiegelmann et al. 2005b). Krall et al. (1982) compared the evolution of the transverse magnetic field component to the motions within a flare-productive AR observed in white light. They found that over a period of several days the observed shearing motion resulted in an ever increasing field alignment with the PIL and was associated to the onset and increase of flaring activity, and which has been supported by the MHD model results of Wu et al. (1984). Force-free modeling results have initiated the search for a possible critical amount of 
shear which, once reached, would inevitably trigger an eruption (Low 1977a, b), but for which observational support seems sparse.

Hagyard et al. (1984b) estimated the critical shear to lie in the range $80^{\circ} \lesssim \theta \lesssim 85^{\circ}$. They defined the shear angle, $\theta$, as the angular difference between the orientation of the transverse field component and the direction perpendicular with respect to the PIL. Consequently, $\theta=0^{\circ}$ implies a field with no shear and $\theta=90^{\circ}$ would account for a transverse field parallel to the PIL. Sivaraman et al. (1992) followed a different approach: they used $\mathrm{H} \alpha$ observations to determine the orientation of the major axis of filaments (as an indication for the direction of the PIL) with respect to an approximation to the orientation of the potential field azimuth. The latter has been defined by the direction perpendicular to a straight connection between the two main spots of the associated bipolar sunspot group. Their study revealed that flares occurred only when the angle between the principal filament direction (i.e., the PIL) and the direction of the potential field azimuth exceeded $\approx 85^{\circ}$ (using the same notation for the shear angle as above).

Another source of instability is the amount of twist of a coronal structure, as induced by systematic motions of the photospheric footpoints of the field. For instance, an equilibrium coronal loop becomes unstable if its length is stretched beyond a critical value, or if it is twisted by photospheric motions (Priest 1978). For twisted magnetic field configurations, different critical thresholds were found within numerical experiments, including uniformly twisted toroidal or periodic, line-tied cylindrically symmetric configurations (Hood and Priest 1981; Baty and Heyvaerts 1996) and locally twisted configurations (Baty and Heyvaerts 1996; Mikić et al. 1990; Fan and Gibson 2003; Török and Kliem 2003). Note, the results of all of these model experiments agree fairly well on the critical amount of twist: the field lines must perform more than one full turn $(T>2 \pi)$ about the center of the flux tube for it to become kink unstable, which would be observed as an eruption under coronal conditions. These numerical considerations also revealed that the critical twist, tends to rise with increasing aspect ratio, that is the ratio of the loop length to its cross-sectional diameter (Einaudi and van Hoven 1983; Török et al. 2004). On the other hand it is smallest for loops of a strong magnetic field, for given temperature and density values (Priest 1978).

\subsection{Magnetic energy budget}

\subsubsection{Energy buildup and storage}

Magnetic flux continuously emerges from where it is generated through the photospheric layers and punches into the pre-existing coronal magnetic field. At the same time, small- and large-scale convective motions in the photospheric layers, shuffle around bundles of frozen-in magnetic field lines. The energy required to perform the work of moving magnetic structures against the ambient magnetic field contributes to the magnetic energy content of the coronal field (Parker 1987). The two major contributions to the transport of magnetic energy to the corona are the emergence of material through the photosphere from below, dragging magnetic field along, and horizontal motions of the photospheric material that increase the shear of the mag- 
netic field above (Wang 1995). The footpoint motions of coronal loops result in an energy flux of $\approx 10^{4} \mathrm{~W} \mathrm{~m}^{-2}$ and $\approx 10^{6} \mathrm{~W} \mathrm{~m}^{-2}$, for typical photospheric quiet-Sun and active-region conditions, respectively, to the corona. This is at least ten times larger than the corresponding radiative and conductive losses in chromosphere and corona (Bellan 2006; Parnell and De Moortel 2012).

The random displacements of the line-tied footpoints of the coronal field-lines naturally lead to "braided" magnetic fields (van Ballegooijen et al. 2014). Such braiding is a measure of how often different field lines cross each other in space Berger and Asgari-Targhi 2009 and is supported by MHD models (Gudiksen and Nordlund 2005; Rappazzo et al. 2008; van Ballegooijen et al. 2011). These braided small-scale field structures efficiently heat the coronal environment by the dissipation of electric currents, where oppositely directed magnetic fields are found in close vicinity to each other (Parker 1983, see also Cirtain et al. 2013; Thalmann et al. 2014). The sub-surface dynamics controlling the temporal evolution of the coronal magnetic field, also launch waves propagating upwards. Currently, from all of the excited types of waves, only Alfvén waves may be capable of actually reaching coronal heights, whereas other types of waves are efficiently damped already in the low atmosphere and thus do not contribute to the heating of the coronal plasma to the observed temperatures see the recent review by Parnell and De Moortel (2012). A discussion about the corresponding coronal heating processes is outside the scope of our review and interested readers might consult also the in-depth discussion by Klimchuk (2006) and dedicated chapters in Cranmer (2009) and Reale (2010).

The time scale on which magnetic energy is transported through the photosphere is much larger than that of the transport through the corona itself. For typical coronal conditions, the travel time over a characteristic coronal length scale is in the order of minutes. For typical photospheric values, on the other hand, one finds characteristic travel times of hours. This implies that the time span during which substantial amounts of magnetic energy are stored in the corona can be long, because sufficiently intense current distributions need to develop.

\subsubsection{Relation to flare productivity}

Depending on the magnetic field topology and its temporal evolution, very different amounts of magnetic energy are stored in time intervals of various lengths. Force-free modeling suggests energy storage rates of a few $10^{23} \mathrm{~J} \mathrm{~h}^{-1}$ to a few $10^{25} \mathrm{~J} \mathrm{~h}^{-1}$ (Thalmann and Wiegelmann 2008; Sun et al. 2012b; Malanushenko et al. 2014; He et al. 2014, and see also Fig. 15a). Besides, such modeling suggests that magnetic energy is stored mainly in the low solar atmosphere, possibly only a few Mm above photospheric levels (Thalmann and Wiegelmann 2008; Sun et al. 2012b, also cf. Fig. 15b).

The size and frequency of eruptive events appears to be related to the portion of the previously stored magnetic energy that is actually available for release at a given time (Choe and Cheng 2000; Jing et al. 2010; Tziotziou et al. 2012). This so-called "free magnetic energy" is the energy difference between a NLFF and a current-free field. One generally finds a higher free energy content prior to larger eruptive events and a small amount of free energy $\left(\lesssim 10^{23} \mathrm{~J}\right)$ prior to the weakest flaring activity (Gilchrist et al. 2012), where "weakest" activity refers to smaller than C-class events. A free 

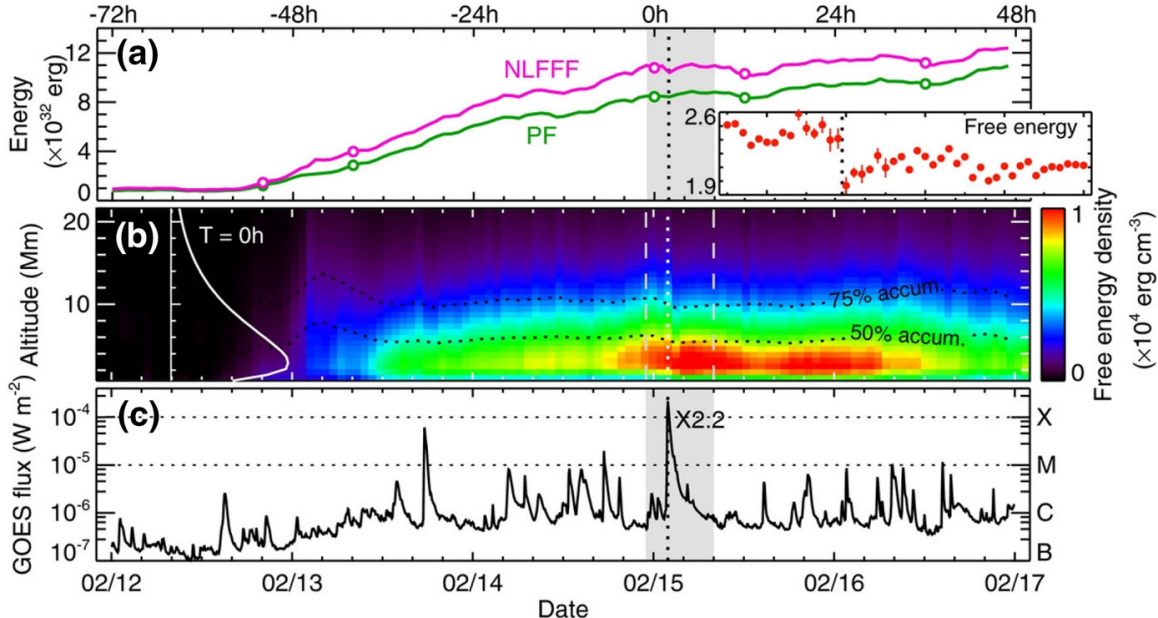

Fig. 15 a Temporal evolution of the total (pink solid line; estimated from a NLFF model) and potential (green solid line) magnetic energy stored in the coronal volume above AR 11158 during 5 days. The inset shows the evolution of the free magnetic energy (that is the excess energy over a current-free field) around the time of an X-class flare (indicated by the vertical dotted line). One can clearly see the more or less continuous increase in magnetic energy, predominately caused by an emerging flux rope $\left(\approx 10^{26} \mathrm{~J}\right.$ during $\approx 48$ h). b Height profile of the associated average free magnetic energy. The white curve shows a sample altitude profile of the average free energy on 15 February at 00:00 UT. The course of each profile over time and over height is visualized by the color code shown. Black, more or less horizontally running, dotted lines indicate the iso-contours of 50 and $75 \%$ of the total free energy. c Measured full-Sun GOES 5-min SXR flux in the 1 to $8 \AA$ channel for the same time range. The vertical, white-dashed lines in $\mathbf{b}$ as well as the gray-shaded area in a and $\mathbf{c}$ mark the duration of an X2.2 flare. The peak time of the flare, on 15 February at 01:56 UT, is indicated by vertical dotted lines. (Adapted from Figure 4 of Sun et al. 2012b. (C) AAS. Reproduced with permission)

energy of $\approx 10^{24} \mathrm{~J}$ to $\approx 10^{25} \mathrm{~J}$ has been reported prior to weak flaring activity (Bleybel et al. 2002; Régnier and Priest 2007; Thalmann et al. 2008; Sun et al. 2012a), where "weak" means C-class flares. Moderate to strong (M- to X-class) events tend to occur only when free energies of some $10^{25} \mathrm{~J}$ to some $10^{26} \mathrm{~J}$ are present (Régnier et al. 2005; Metcalf et al. 2005; Thalmann and Wiegelmann 2008; Jing et al. 2009; Sun et al. 2012a; Feng et al. 2013). Note, however, that the buildup of a sufficient amount of energy on its own does not guarantee the occurrence of a subsequent eruption and the release of part of this energy (Gilchrist et al. 2012).

\subsubsection{Energy release}

The release of magnetic energy is believed to take place in current sheets, very limited regions where magnetic field and plasma are locally decoupled. This allows the magnetic field to diffuse and change its topology by means of magnetic reconnection (see Sect. 4.2.2). It has been contended by Moore et al. (1995) and Lin et al. (2009) that the width of these regions is $\approx 0.1$ to $1 \mathrm{Mm}$. Narrower current sheets, as proposed by Wood and Neukirch (2005), would result in even smaller diffusion time scales. Clearly, unlike the global magnetic diffusion time-scale of several months, the diffu- 
sion time scale in currents sheets is very small ( $\tau_{d} \approx 1 \mathrm{~s}$ to $\left.10 \mathrm{~min}\right)$. In other words, the presence of current sheets in the corona, permits the efficient release of magnetic energy on active-region scales, in contrast to the non-efficient magnetic diffusion on global scales. Due to the rarity of vector magnetic field measurements in the corona, direct observational detections of current sheets are restricted basically to the upper chromosphere. From observations, only an upper limit of roughly $1 \mathrm{Mm}$ can be given on the width of current sheets (Solanki et al. 2003).

Force-free models of ARs indicate that the height where the gross part of the magnetic energy is released is located $\lesssim 20 \mathrm{Mm}$ above the photospheric level (Thalmann and Wiegelmann 2008; Sun et al. 2012b). Only a fraction of the released free energy goes into the acceleration of particles. Heated and/or accelerated, particles propagate from the reconnection site, near the apex of the coronal loop, downwards along the legs of the loop to the chromosphere, where they lose their energy due to collisions with the denser chromospheric material. This, in turn, is heated and convected upwards and fills the newly reconnected field lines with chromospheric and transition region plasma heated to coronal temperatures, emitting in SXRs (Neupert 1968).

Just as the free energy content prior to observed eruption is related to the flare class, one would expect that the actually released amount of free energy relates to the flare class too. Recent studies, however, do not show a clear tendency, i.e., no clear trend of more energetic events consuming more of the previously stored energy. So far, the estimated upper limits for the decrease of free magnetic energy range from 5 to $50 \%$ (Thalmann and Wiegelmann 2008; Thalmann et al. 2008; Jing et al. 2009; Sun et al. 2012b; Feng et al. 2013). Cases were also reported without a clear decrease (Sun et al. 2012a) or even an increasing free energy (Metcalf et al. 2005, and references therein). However, there have been doubts about the reliability of the latter owing to very uncertain estimates. Often also a decrease of the free energy occurs already before the time of the peak SXR flux of a flare, and in other cases this has been seen even before the flare onset (Jing et al. 2009; He et al. 2014).

Recently, Feng et al. (2013) investigated the partition of magnetic energy during an $\mathrm{X}$-class and CME event. The radiative energy release estimated from the recorded flare emission was found to be at least one order of magnitude lower than the upper limit of available free magnetic energy; this also held for the kinetic and potential energy of the associated CME and confirmed the general consensus that by far not all of the free energy is released even during the most energetic events, but that a considerable portion remains available to power successive eruptions (see also Fig. 19). As discussed for the helicity budget on a global scale (see Sect. 3.4), a non-potential magnetic field is never found to relax to a corresponding current-free configuration, in the course of an eruption. Despite expected in terms of helicity conservation, a non-potential coronal field may never entirely relax to a corresponding constant-alpha (preserved helicity) state due to the line-tied nature of the coronal structures (Antiochos and DeVore 1999). Besides, newly emerging flux also permanently disturbs the relaxation process.

\subsubsection{Coronal implosion and photospheric response}

The release of magnetic energy naturally leads to a deviation from the previous balance of the magnetic forces in an AR. The magnetic pressure is directed towards 
(a)

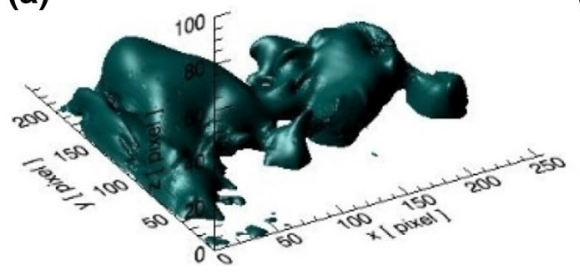

(b)

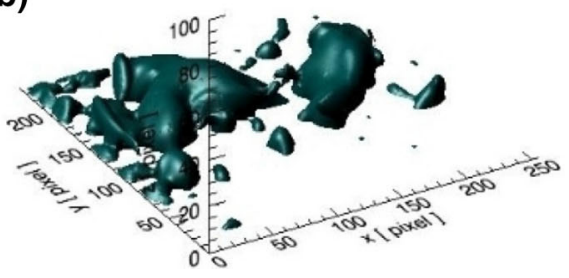

Fig. 16 NLFF magnetic field model of the active-region field above NOAA AR 10540 a before and $\mathbf{b}$ after a large M-class flare. Iso-surfaces of a absolute magnetic field strength of $200 \mathrm{G}$ are shown. The iso-surfaces give an impression of the magnetic pressure, sustaining different parts of the AR. It is evident that various areas within the AR react with different sensitivity to the flare, i.e., show differently strong signatures of implosion. (Adapted from Figure 3.7 of Thalmann 2010. Reproduced with permission)

weaker magnetic fields, i.e., generally pointing upwards towards higher altitudes and competes with the magnetic tension that tries to reduce the curvature of the magnetic field lines. The rapid release of energy during an eruption may cause a reduction of magnetic pressure, accompanied by a deflation of the magnetic field, termed "coronal implosion" (Hudson 2000, and see Fig. 16). Model calculations by Janse and Low (2007) confirmed that if the post-eruption thermal pressure cannot compensate for the eruption-related magnetic pressure reduction, a magnetic system will implode.

Frequently, observational evidence for such implosions is found in the form of the contraction of observed coronal loops (Liu et al. 2009a; Liu and Wang 2009; Sun et al. 2012b; Gosain 2012; Simões et al. 2013). Liu et al. (2012) claimed that the apparent loop contraction is not just a projection effect. This conclusion, they argued, was possible due to their observation of the contracted coronal loops to reside at lower heights than those of the pre-flare system (see Fig. 17 and also Liu and Wang 2010). Importantly, the magnetic field lines after an eruption never regained their pre-flare heights. Almost all of the studied eruptions showed both contracting overlying largescale loops that are observable in cooler EUV channels, and expanding components underlying, newly reconnected field lines, preferentially visible in warm/hot EUV channels. However, even before the conventional large-scale expanding motions could be seen for particular flares, contracting motions during the impulsive phase of flares were observed in the form of descending motions of loop-top sources (Krucker et al. 2003; Joshi et al. 2009) and EUV flaring loops (Li and Gan 2006; Liu et al. 2009a), along with converging $\mathrm{H} \alpha$ ribbons or HXR footpoints. Ji et al. (2007) explained the observational aspects related to the initial contraction in terms of the relaxation of a modeled highly sheared core field. They formed new field lines, still highly sheared and containing much of the non-potential energy. The dissipation of free energy causes the sheared field to relax and contract; their effect is much stronger than the expansion effect by reconnecting arcade field lines during the initial phase.

The deflation of coronal loops, caused by the release of magnetic energy, is often also accompanied by changes of the shear of the horizontal component of the photospheric magnetic field. The sign of this change remains inconclusive, however, since both, an increase of the shear (Wang 1992; Chen et al. 1994; Wang et al. 1994, 2012a, b; Liu et al. 2005; Petrie 2012), as well as a decrease has been found during flares (Wang 2006). 

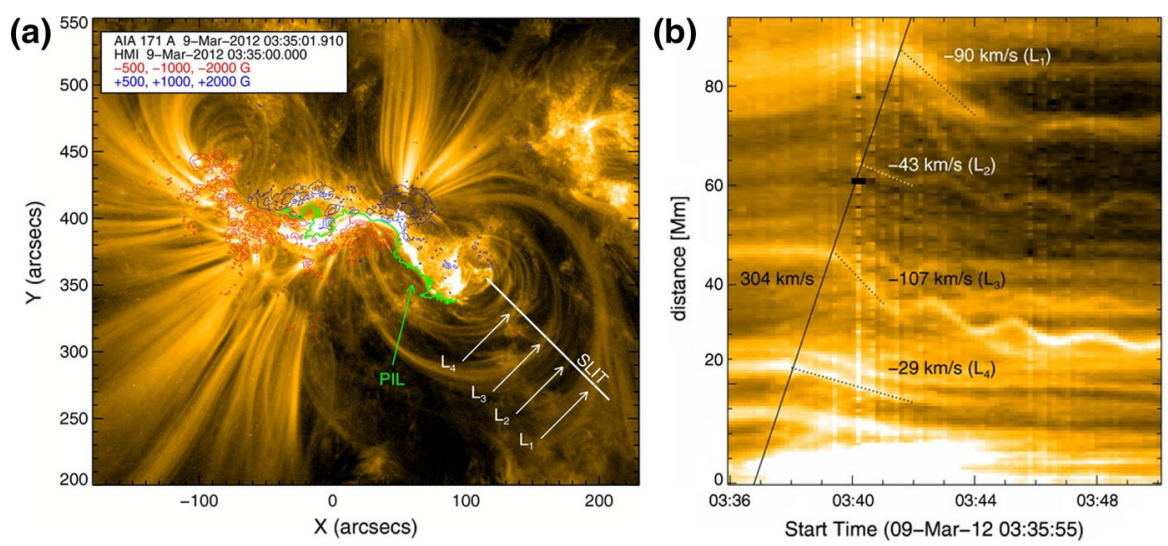

Fig. 17 a SDO/AIA image of the $171 \AA$ emission of NOAA AR 11429 on 9 March 2012. The blue/red contours resemble the co-temporal negative/positive LOS component of the photospheric magnetic field measured with SDO/HMI. The green line marks the PIL. The white line indicates the slit along which the motion of four well-defined bundles of coronal loops $\left(\mathrm{L}_{1}\right.$ to $\left.\mathrm{L}_{4}\right)$ is investigated. b Time-position diagram during the impulsive phase of an M6.4 flare. Shown is the projected speed of the coronal loop bundles which contract with different apparent speeds. A delay of the implosion onset with height is clearly visible and indicated by the inclined black straight line. (Adapted from Figures 1 and 2 of Simões et al. 2013. (C) AAS. Reproduced with permission)

To explain such contradictory results, Dun et al. (2007) proposed that the measures for the non-potentiality of a magnetic field may take on different values from one portion of an AR to another. Findings may then depend on the specific choice of the analyzed photospheric area. Moreover, projection effects due to the location of the investigated area on the solar disk might also result in changes of the observed longitudinal field, similar to that expected for flare-related reconfigurations (Wilkinson et al. 1989; Venkatakrishnan et al. 1989; Spirock et al. 2002). We also point out the need to check carefully to what extent the determined magnetic field configuration is affected by the thermal and velocity structure of the atmosphere (see review by Solanki 1993) by weak blends in the employed spectral lines, or by instrumental effects. Finally, as recently speculated, the magnetic field may behave differently at different altitudes with varying domains of increasing and decreasing shear (Jing et al. 2008; Sun et al. 2012b). No unique mechanism accounting for all of these observed aspects of magnetic shear has so far been identified.

\subsection{Magnetic helicity budget}

The storage of magnetic energy in the coronal volume above ARs is accompanied by an accumulation of magnetic helicity (see Sect. 3.4 for the Sun's global helicity budget and Sect. 5.2.6 for a corresponding discussion on quiet-Sun fields). Often, also the current helicity is investigated, this quantifies how much the fields are locally twisted (Démoulin 2007). In contrast to magnetic helicity, the current helicity is not a conserved quantity; the general relationship between the two is not known (van 
Driel-Gesztelyi et al. 2003). Within this work, we restrict ourselves to the discussion of magnetic helicity.

\subsubsection{Helicity buildup and storage}

Any magnetic helicity gained by a dynamo action somewhere within the convection zone during the generation of magnetic field is transported into the solar atmosphere by the emergence of helical magnetic flux tubes in the photosphere (Seehafer 1990). The flux of magnetic helicity at photospheric levels is the result of the combined effect of the motions of magnetic structures due to convective plasma flows and the subsequent advection of these helicity-carrying structures. For a review on observations and modeling of the helicity at photospheric levels, see Démoulin and Pariat (2009). The photospheric motions that twist and/or shear the magnetic flux tubes result in the coronal magnetic field to gain even more helicity.

Investigating the helicity injection rate in ARs by shearing motions only requires the knowledge of the normal component of the magnetic field and the horizontal velocity of the magnetic elements within an AR (Berger 1984; Démoulin and Berger 2003; Chae et al. 2004). The resulting estimate is thus a mixture of the enhancement of the helicity content by shearing motions and the portion of helicity emerging from below the photosphere. Magara and Longcope (2003) proposed that the helicity contribution from the vertical flows might only dominate early phases of flux emergence, followed by a dominant contribution from the shearing flows in the later stages of an evolving AR. This is in the opposite of what has been presented recently by Liu et al. (2014) who analyzed the ARs during their emergence phases and found that shearing motions contribute the most to the ARs' helicity content.

And also other contributions to the helicity budget within an AR were detected, including the rotational motion of sunspots around their own axis, which results in enhanced magnetic twist. The rotation of the center of one polarity around the center of the reversed polarity center also adds to the writhe of a magnetic flux tube's axis (Tian and Alexander 2006; Tian et al. 2008; Kumar et al. 2013). Liu and Zhang (2006) estimated the accumulation of helicity associated to the rotation of a pair of bipolar sunspots (during about $31 \mathrm{~h}$ ) to be $\approx 2 \times 10^{42} \mathrm{Mx}^{2}$. They noted another increase of $\approx 3 \times 10^{43} \mathrm{Mx}^{2}$ during a following period of $\approx 96 \mathrm{~h}$, while strong shearing motions were observed. Zhang et al. (2008) estimated the helicity injected by the rotational motion of sunspots around their centers within an AR to be $\approx 10^{43} \mathrm{Mx}^{2}$, which was on the same order as the combined contribution by shearing motions and flux emergence.

The above discussion underlines that further detailed research on evolving ARs is needed to disentangle the contribution of emerging, shearing and rotational motions and to assess their importance for the total helicity content of ARs. Importantly, Vemareddy et al. (2012b) showed that such estimates depend on the time interval between successive magnetic vector maps and the window size within which the horizontal footpoint velocities are tracked. Correspondingly, they guessed the general uncertainty of calculated helicity injection rates and total helicity accumulations to be $\approx 10 \%$. Vemareddy et al. (2012a) found evidence that individual portions of an AR contributed differently to the total helicity content. While in one part an accumulation of negative helicity was recorded, an injection of positive helicity was found in another 


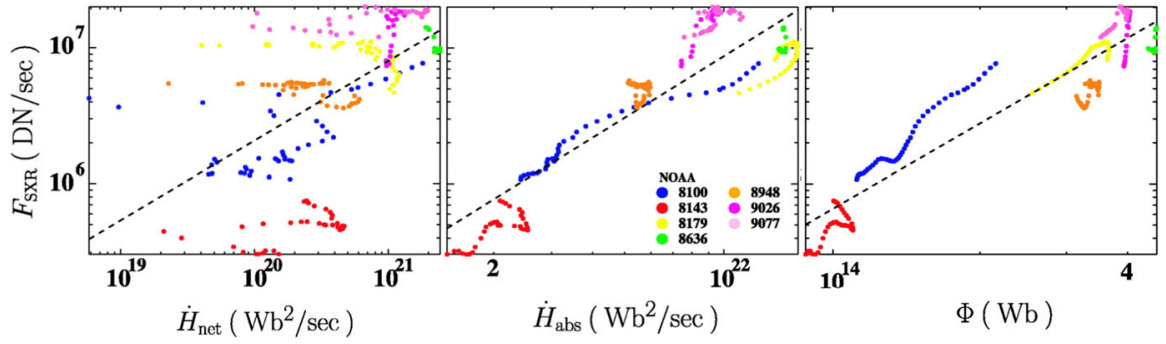

Fig. 18 Time-averaged a net helicity flux, $\dot{H}_{\text {net }}$, b absolute magnetic helicity flux, $\dot{H}_{\text {abs }}$, and c unsigned magnetic flux, $\Phi$, as a function of the SXR flux, $F_{\mathrm{SXR}}$, of seven ARs. The SXR flux is estimated from the measured intensity Yohkoh/SFD images by integration over the area used for helicity calculations and corresponds to the Yohkoh data number (DN) per second, where $1 \mathrm{DN}$ corresponds to 100 electrons in the CCD detector. Time averages are taken over $24 \mathrm{~h}$. Values corresponding to NOAA ARs 8100, 8143, 8179, 8636, 8948, 9026, and 9077 are represented by blue, red, yellow, green, orange, magenta, and pink dots, respectively. The dashed line represents a regression line, obtained from a least-squares method. One can see that the absolute helicity flux correlates better with the SXR flux than does the net helicity flux for the individual ARs. Note that for all ARs except 8143 and 8636 repeated flaring activity (C-, M- or X-class) has been reported. (Adapted from Figures 3 and 8 of Maeshiro et al. 2005. (C) AAS. Reproduced with permission)

part. Vemareddy et al. (2012b) recognized that major flaring activity only occurred at times when helicity was injected in the AR with a sign opposite to the dominant sign of the AR (see also Kusano et al. 2002). Similar findings have been presented for CME(Wang et al. 2004) and MC-productive (Chandra et al. 2010) ARs. For these too, the sign of the helicity inherent to the newly emerging magnetic flux systems may well be opposite to that of the dominant helicity in the pre-existing active-region magnetic field.

\subsubsection{Relation to flare productivity}

The rates of injected magnetic flux and helicity are necessarily related to each other (LaBonte et al. 2007; Tian and Alexander 2008; Park et al. 2008; Chandra et al. 2009; Tziotziou et al. 2012). The flare productivity, however, may be more closely related to magnetic helicity injection than to the amount of injected magnetic flux (or stored magnetic energy). More precisely, if positive and negative helicity is injected at the same time at approximately the same rate in flare-productive ARs, the absolute helicity flux (transported into the atmosphere by emerging motions) correlates well with the SXR flux (Maeshiro et al. 2005 and see Fig. 18). Park et al. (2010) based on a similar conclusion on the observation that for flaring ARs with a large unsigned magnetic flux, the average helicity injection rate was twice that of non-flaring regions. In fact, a systematic study by LaBonte et al. (2007) on the helicity injection in ARs showed that all X-class flares were associated to peak helicity fluxes greater than $6 \times 10^{36} \mathrm{Mx}^{2} \mathrm{~s}^{-1}$ and consequently proposed a causal link of the peak helicity flux to the ability of an AR to produce an X-class flare.

This proposal raises the question whether or not there is a "critical" amount of helicity which, once reached, favors the onset of an eruptive process. Park et al. (2008) 
found a nearly constantly increasing coronal helicity, preceding all of the considered 11 $\mathrm{X}$-class flares. Importantly, they suspected an individual critical limit of accumulated helicity for each of the eruptive ARs, since they could not identify a common threshold for all events. Park et al. (2010) compared the magnetic properties and flaring activity for a sample of 378 ARs. They note that prior to all flares with a peak SXR flux of more than $5 \times 10^{-5} \mathrm{~W} \mathrm{~m}^{-2}$, a significant amount of helicity $\left(10^{42}\right.$ to $\left.10^{43} \mathrm{Mx}^{2}\right)$ was monotonically accumulated during $12 \mathrm{~h}$ to $48 \mathrm{~h}$. Analyzing more than 150 ARs, Tziotziou et al. (2013) found that a relative helicity of $\approx 10^{42} \mathrm{Mx}^{2}$ and $\approx 10^{43} \mathrm{Mx}^{2}$ is accumulated prior to $\mathrm{M}$ - and X-class flaring, respectively. Note that whenever we speak of "relative" helicity, the helicity of a non-potential field with respect to that of the corresponding potential field is meant. Earlier already Tziotziou et al. (2012) had estimated the instantaneous magnetic energy and helicity budgets of ARs and found that those with a free energy of $\lesssim 4 \times 10^{24} \mathrm{~J}$ and a relative helicity of $\lesssim 2 \times 10^{42} \mathrm{Mx}^{2}$ were not flare productive. Also, the flare class of the observed events was successively smaller (X-to C-class) for ARs with successively less free energy and relative helicity budgets. Early estimates of the helicity budget of flare and CME-productive ARs, based on force-free field models, range from $\approx 10^{34} \mathrm{Mx}^{2}$ to $\approx 10^{43} \mathrm{Mx}^{2}$, with the general tendency that ARs with a higher helicity content are more flare productive (Régnier et al. 2002; Régnier and Canfield 2006; Régnier and Priest 2007).

In recent years, considerable effort has been invested into developing methods that infer the coronal helicity budget based on the reconstructed NLFF coronal magnetic field. This is far from trivial, since it often involves the computation of the magnetic vector potential that has to satisfy specific gauge properties (Rudenko and Myshyakov 2011; Thalmann et al. 2011; Valori et al. 2012a; Yang et al. 2013). So far, these models have not been applied to real solar cases, but have been tested by the use of semianalytic force-free magnetic field solutions. Only Valori et al. (2012b) investigated the helicity content of an AR during flux emergence and found a relative helicity of $\approx 10^{42} \mathrm{Mx}^{2}$ prior to background, i.e., B-level flaring activity. The hesitation to apply the developed methods certainly arises from the strong influence the choice of a particular gauge as well as its numerical implementation may have on the model outcome, especially when applied to real data. Only when the reliability of such methods has been assessed, will it become clear, whether summing over the helicity injected at a photospheric level, i.e., estimated from routine direct photospheric magnetic field measurements, represents the safest estimate of the coronal helicity content (Démoulin and Pariat 2009).

\subsubsection{Helicity dissipation and transport}

In contrast to dissipating magnetic energy, magnetic reconnection is a very inefficient process for removing magnetic helicity. This was shown by Berger (1984), who had tested the magnetic helicity decay in a coronal loop, by assuming classical anomalous conductivity in a circular loop with a length of $100 \mathrm{Mm}$, a radius of $10 \mathrm{Mm}$, and an uniform temperature of $1 \mathrm{MK}$. He found a diffusion time scale of $\approx 10^{9}$ to $10^{13} \mathrm{~s}$, which is far too long to be relevant for time scales of impulsive (eruptive) processes such as flares, where typical time scales are $\approx 10^{3} \mathrm{~s}$. Instead, during magnetic reconnection, and on time scales relevant for it, the magnetic helicity is approximately conserved. 
Only its source, the mutual linking of different flux systems or the internal twist of a magnetic structure, is transformed (Hornig and Rastätter 1997). This provides a constraint on the relaxation of a current-carrying magnetic-field configuration by energy dissipation: it can only relax to another lower-energy, still current-carrying state but not to a current-free one (Woltjer 1958; Taylor 1974; Bellan 1999, 2006). Importantly, the decrease of both magnetic helicity and of magnetic energy due to an eruption is often temporary and sets in before the onset of the eruptive event, as defined by the impulsive rise of SXR emission (Jing et al. 2009; Tziotziou et al. 2013). Both are often found to return to, or even exceed the pre-eruptive level within hours, thus allowing for subsequent flaring activity (see Fig. 19).

As a consequence of the conservation properties of magnetic helicity, a change of the coronal magnetic helicity content can only be caused by an expulsion of a magnetic structure and its inherent helicity, such as a CME to interplanetary space. A typical CME contains a magnetic helicity in the order of $10^{42} \mathrm{Mx}^{2}$, as this is also a the typical magnetic helicity content of a MC (DeVore 2000; Georgoulis et al. 2009, and see also Sect. 3.4). These estimates agree very well with the helicity budget of ARs prior to major eruptions ( $\gtrsim 2 \times 10^{42} \mathrm{Mx}^{2}$; see Tziotziou et al. 2012, and see Sect. 4.6.1). MCs often show the same chirality as their source region, as inferred from the twist of the flux tubes they originate from (Rust 1994; Mandrini et al. 2004). But events have also been reported where this has not been the case. For instance, Chandra et al. (2010) investigated a case in which observed features (sunspot whorls and flare ribbons) as well as a corresponding LFF magnetic field suggested a predominantly negative helicity within an AR. In contrast, the associated MC was attested a positive helicity, which contradicted helicity conservation. Only a very accurate analysis of the helicity injection revealed that a strong, local injection of positive helicity served as the source for the observed positive helicity of the MC.

Note that already Kusano et al. (2004) outlined the importance of neighboring flux systems of helicity with opposite signs within a single AR for its flare productivity, and Leamon et al. (2004) attested only models invoking magnetic reconnection between the small-scale active-region magnetic fields and their overlying large-scale envelope field, where the latter ejected an MC, the ability to relate their helicity aspects correctly.

\section{Quiet-Sun magnetic fields}

Traditionally the "quiet-Sun" regions got their name because they were assumed to lack activity. However, while large-scale eruptions are mainly associated with ARs (see Sect. 4), the QS is not quiet either. Dynamic processes that occur on smaller scales had simply not been resolved by early missions and instruments. Already Labonte (1979) pointed out that the total magnetic flux of the QS exceeds the flux contained in ARs by far and that it drives activity, such as spicules. These were first observed in $\mathrm{H} \alpha$ and described as "small spices of chromospheric material" (Roberts 1945). Ephemeral ARs (see Sect. 1.1.1) were also found to be centers of activity Harvey and Martin (1973). As in their active-region counterparts, the photospheric magnetic field in the ephemeral regions drives activity, such as X-ray bright points, (Golub et al. 1974, 1977; Priest 1994) in the upper solar atmosphere. 


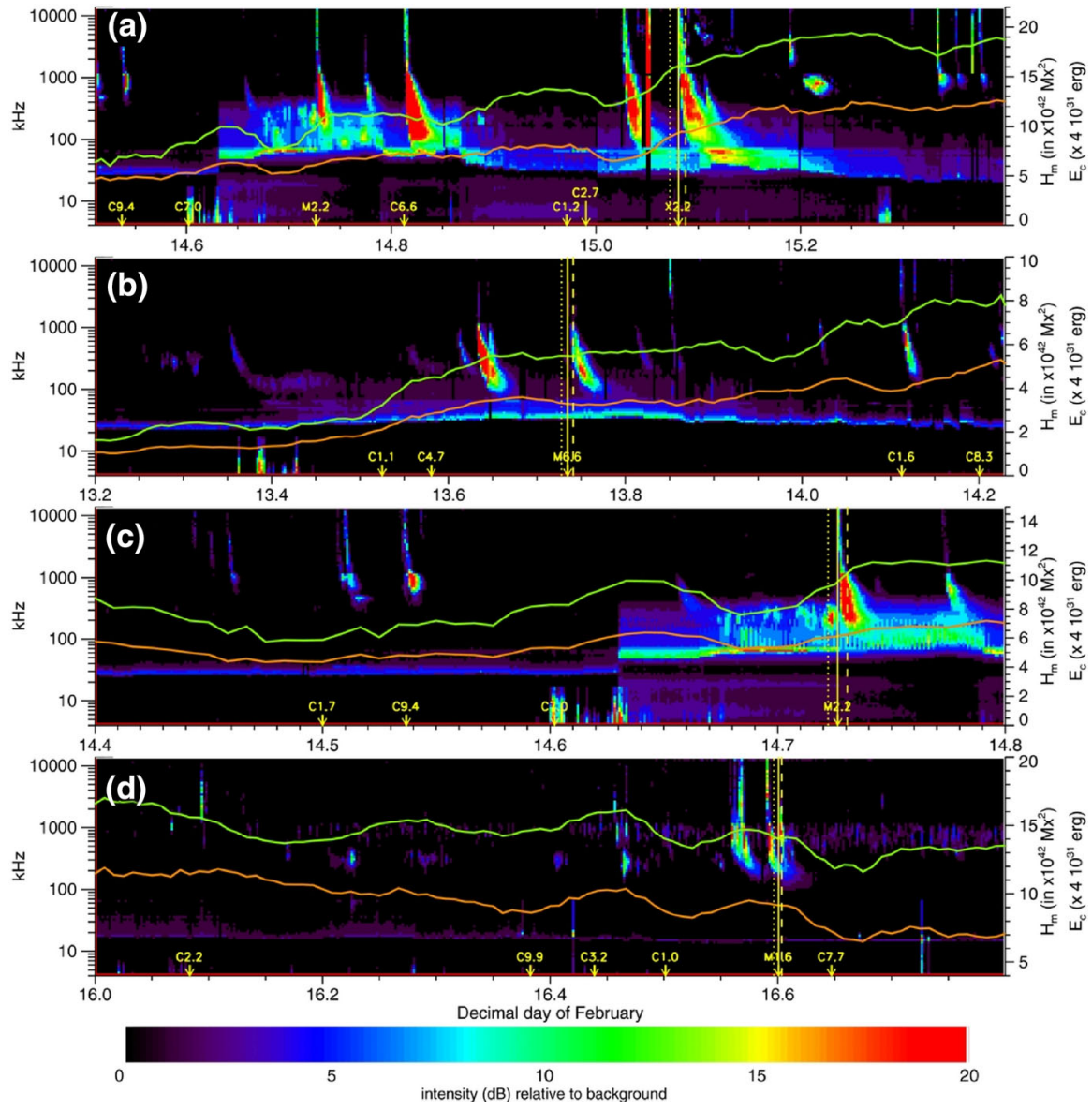

Fig. 19 Relative helicity (green curves) and free magnetic energy (orange curves) of AR 11158 in February 2011, around the times of four large eruptive events (with values are averaged over $72 \mathrm{~min}$ ): a an X2.2 flare of 15 February, $\mathbf{b}$ an M6.6 flare on 13 February, $\mathbf{c}$ an M2.2 flare on 14 February, and $\mathbf{d}$ an M1.6 flare of 16 February. The flare onset, peak and end times (defined by the GOES SXR flux) are indicated by dotted, solid and dashed vertical lines, respectively. The color-coded background resembles the cotemporal WIND/WAVES frequency-time radio spectra. It can well be seen that a decrease of the magnetic energy and relative helicity magnitude starts before the onset of the flares and displays a gradual, rather than instantaneous character. (Figure 11 of Tziotziou et al. 2013. () AAS. Reproduced with permission)

\subsection{D magnetic field structure in the quiet Sun}

Because of the often mixed magnetic polarities on smaller scales in the QS, we mainly find magnetic loops which are very short and hardly reach into higher layers of the solar atmosphere. With modern high-resolution measurements, however, we can use magnetic field modeling techniques to model the 3D structure in the QS, similar to those described in Sect. 2.2 and which are routinely applied to the active Sun (see Sect. 4). Nevertheless, some care has to be taken, as is discussed in Sect. 5.2.5. 
Because of their easier computational load and because only photospheric LOS measurement are required as boundary condition, the topology of the quiet Sun's outer atmosphere and its temporal evolution is usually investigated by use of potential field models. However, one has to keep in mind that any static-model approach provides only a snapshot of the, in reality dynamic, magnetic carpet. Schrijver and Title (2003) modeled the fraction of magnetic flux that connects from network elements into the upper solar atmosphere and found it to scale with the flux density of the underlying internetwork field. For relatively strong internetwork flux densities, in the order of $5 \times 10^{-3} \mathrm{~Wb} \mathrm{~m}^{-2}$, only about 30 to $40 \%$ of the flux connects to the corona while the greater part connects back down in the form of closed loops.

\subsubsection{Photospheric quiet-Sun loops}

The structure and dynamics of the quiet-Sun magnetic field can be investigated with spectro-polarimetric measurements. Martínez González et al. (2007), using data from VTT, found low-lying photospheric magnetic loops connecting about 10 to $20 \%$ of the visible magnetic flux of opposite polarity. Note that emerging small-scale loops are observed in the form of newly detected horizontal fields well before vertical flux is detected (see Centeno et al. 2007, for a corresponding study based on Hinode/SOT-SP data). In subsequent studies, Martínez González and Bellot Rubio (2009) and Martínez González et al. (2010) presented observations of emerging $\Omega$-loops by combining magnetic and imaging data from Hinode/SOT and DOT. With an even higher resolution, based on the balloon-borne Sunrise/IMaX instrument, Danilovic et al. (2010a) investigated a large number of transverse photospheric features. The high-resolution IMaX data revealed that $97 \%$ of these features are smaller than the mean size of such structures $\left(\approx 1^{\prime \prime}\right.$.0 squared) found in earlier studies.

Already Isobe et al. (2008) had detected emerging $\Omega$-shaped loops. They showed that such structures, after emergence, reach the chromosphere and reconnect with the mainly vertical fields there. Thus they contribute to the heating of the chromospheric plasma there and to generating coronal MHD waves. These high-frequency waves may then contribute to coronal heating and the acceleration of the solar wind particles. For a description of the kinetic processes involved in coronal heating, we refer to specialized reviews such as that by Marsch (2006).

\subsection{Associated photospheric fields and the response on the upper atmosphere}

QS structures are quite dynamic, as new magnetic flux permanently emerges, usually in bipolar form. The corresponding loops connecting the two polarities emerge into the solar atmosphere, while the loop footpoints move apart due to the shape of the rising loop. The footpoints also undergo displacement due to horizontal photospheric motions. The opposite polarity footpoints keep separating until eventually one or both cancel with older photospheric magnetic flux (Martin et al. 1985). As a consequence, the magnetic flux in the quiet-Sun photosphere is replaced every $\approx 14 \mathrm{~h}$ (Hagenaar 2001). An important question is, how the corona responds to these changes in the photospheric field and how we can get insight into the quiet-Sun field in the upper 
solar atmosphere. This was addressed by Close et al. $(2004,2005)$ using potential field modeling based on photospheric SOHO/MDI LOS magnetograms. They found that the coronal magnetic field becomes redistributed even faster, with a typical time of $1.4 \mathrm{~h}$.

\subsubsection{Doubts about the concept of a magnetic canopy}

The concept of a magnetic canopy (Gabriel 1976, and see Sect. 1.3.1), in the sense of a horizontal magnetic field that fills the upper chromosphere and overlies a most field-free atmosphere, is still a subject of debate. One problem is that there are multiple definitions of a canopy. One is that it is nearly horizontal field overlying largely fieldfree regions. Another is that it is a horizontal boundary between the $\beta>1$ and $\beta<1$ field. Instead of vertically more or less well-divided domains of high or low $\beta$, such domains may well be mixed up, even "islands" may exist (Rosenthal et al. 2002).

A number of comparative studies of magnetic field structures and/or bright emission from photospheric levels, and corresponding signatures from higher atmospheric layers, revealed no obvious expansion of the magnetic network with height (see Sect. 1.3). Zhang and Zhang (2000a,b), for instance, compared quiet-Sun magnetic signatures inferred from Fe I $5324 \AA$ (photosphere) and $\mathrm{H} \beta 4861 \AA$ (chromosphere) observations and found no significant change of the magnetic configuration over a height of $\approx 1.5 \mathrm{Mm}$. They emphasized, however, that the $\mathrm{H} \beta$ line exhibits high noise levels so that the expansion of the magnetic features might simply be hidden by the noise. Peter (2002), among others, presented SOHO/SUMER CI continuum (chromosphere) and O VI $1037 \AA$ (transition region) brightness observations which revealed network elements of nearly the same size. This observation can be construed to contradict the concept of a nearly horizontal canopy at a height of $\approx 1 \mathrm{Mm}$ above the photosphere, or alternatively, it implies that the brightness structures may expand less rapidly with height than the magnetic field. Using TRACE 171 Å observations, already Zhang et al. (1999) had found that the width of the emission associated to fibrils was nearly the same at different heights: at their photospheric root as well as $\approx 30 \mathrm{Mm}$ higher up in the atmosphere. It was speculated as a possible explanation that, either the observed emission might not be sensitive to the detection of the desired canopy structure-as recently supported by Peter and Bingert (2012), see Sect. 1.4.2 for details—or that network fields in the QS could, by some mechanism, be prevented from fanning out (Zhang 2005). These claims would imply that the need for an extra force, at least as strong as the horizontal pressure gradients, that prevents the magnetic field from expanding with height. At least in photospheric layers, magnetic structures and the bright points associated with them are seen to expand with height (Briand and Solanki 1995; Bühler et al.2014, Astron. Astrophys., submitted).

Schrijver and van Ballegooijen (2005) modeled the quiet-Sun network magnetic field at heights of $\lesssim 20 \mathrm{Mm}$ above chromospheric levels. They found an average plasma- $\beta$ close to unity in the entire simulation domain, indicating that the quietSun corona is not force-free, i.e., that the magnetic field there is not unaffected by the plasma. A magnetic canopy also suffers at the hands of atmospheric flows. Thus, Pietarila et al. (2011) demonstrated on the basis of 3D radiation MHD models that in particular downflows pull U-loops out of a magnetic canopy into the lower lying 


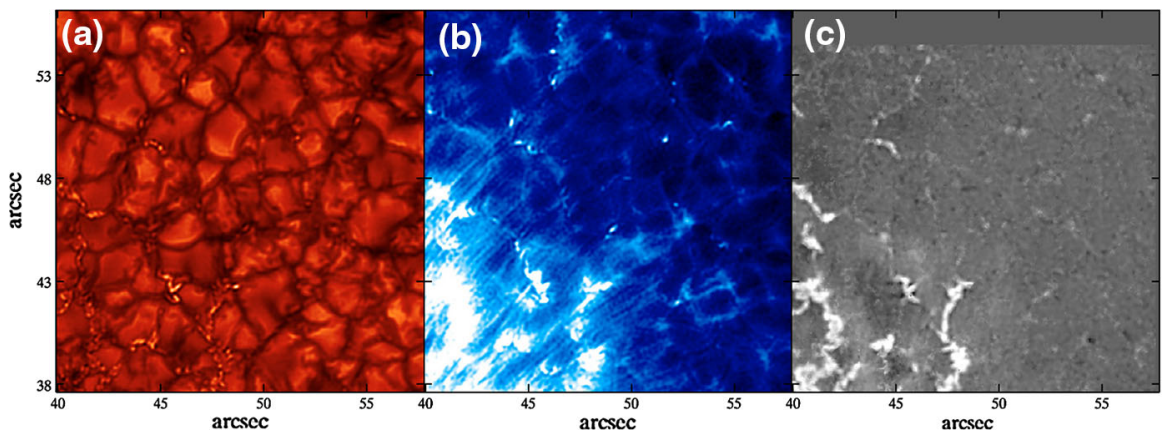

Fig. 20 a Continuum image, b CaII K intensity and c longitudinal magnetic flux (ranging from $-800 \mathrm{G}$ to $1 \mathrm{kG}$ ) on 9 August 2007, covering a plage region and the QS seen as bright and dark areas in b, respectively. The continuum image displays a clear pattern of dark intergranular lanes, often exhibiting emission from more or less tightly packed bright photospheric filigree. Comparison with the Ca II $\mathrm{K}$ image shows that many of the observed thin chromospheric fibrils originate from the locations populated with filigree. Longer fibrils, originating near the boundary between the bright plage and surrounding (darker) QS areas indicate a canopy-like magnetic connection between areas of stronger, i.e., plage, and weak, i.e., QS, magnetic fluxes. (Figure 8 from Pietarila et al. 2009. Reproduced with permission, (C) ESO)

atmosphere, giving rise to some of the horizontal fields seen in the quiet photosphere. With time the canopy loses its clear identity due to this process.

The canopy separates the fields originating from the network and supposedly opening into the atmosphere from closed fields, originating and closing in the weak internetwork. In a numerical experiment, Schrijver and Title (2003) showed that strong internetwork fields allow flux densities capable of considerably modifying the dynamic geometry of the magnetic coupling between the photosphere and outer layers. They argue that bunches of field lines likely connect from strong internetwork regions well up to the corona while field lines from the network close back onto internetwork fields. They estimated that the magnetic flux which originates from internetwork fields and connect to the corona to be $\approx 50 \%$ of the total flux of the quiet-Sun coronal magnetic fields. It may well be that in the mixed-polarity QS, canopies play a less important role than in the more unipolar environment of CHs (see Fig. 27 in Sect. 6.2.2), which is where they are generally placed in theoretical studies.

\subsubsection{Network and internetwork magnetic loops}

Wiegelmann et al. (2010a) used a potential and LFF magnetic field model and found that the majority of the model field lines connect strong internetwork features below equipartition field strength with network elements. They were also able to show that for most of the field lines reaching chromospheric and coronal heights, one of the footpoints is located in the strong internetwork fields. In other words, while the idea of a canopy, formed by horizontal fields above activity centers, seems well established it is more controversial for the atmosphere above quiet-Sun regions. However, observational evidence was found by Pietarila et al. (2009) that chromospheric fibrils formed a canopy over parts of a plage region and the quiet-Sun network (see Fig. 20). They point out that this might not even contradict the finding of Schrijver and Title (2003) - 
(a)

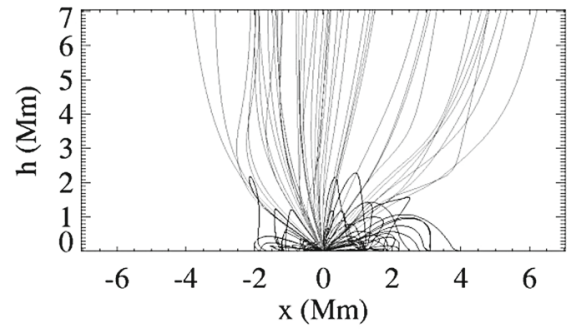

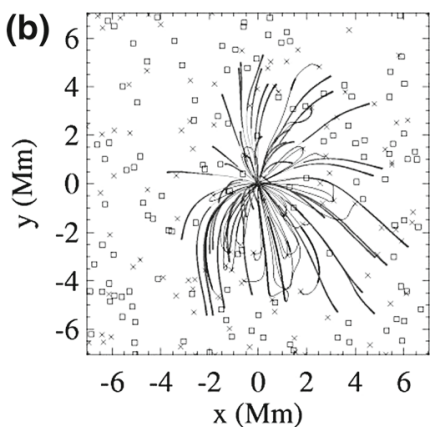

Fig. 21 Illustration of the magnetic carpet, computed from a potential field model, when viewed a from the side and $\mathbf{b}$ from top. The plot depicts a photospheric magnetic flux concentration with a flux of $3 \times 10^{8} \mathrm{~Wb}$, surrounded by a small-scale mixed-polarity field (marked by squares and crosses for opposite polarities). The FOV reaches halfway to the neighboring network sources. Field lines with one footpoint in the central flux concentration (closed field lines shown as black and open field lines shown as gray curves) suggest that a collar of field lines that emanates from the network and closes back onto the internetwork field within several Mm, implying that most of the coronal field is likely anchored in the internetwork field, rather than in the network. (Adapted from Figure 1 of Schrijver and Title 2003. ( A AAS. Reproduced with permission)

namely, that internetwork fields disable the formation of a large-scale canopy-but instead, that the apex of small-scale loops of the internetwork may not always reach a height in the atmosphere where a canopy might exist. And indeed, potential and forcefree magnetic field extrapolations carried out by Wiegelmann et al. (2010a) showed that the number of short field lines (with apex heights of $\lesssim 500 \mathrm{~km}$ ) outnumber the field lines reaching the corona (with apex heights $\gtrsim 2.5 \mathrm{Mm}$ ). It has to be noted, however, that a rather small portion of the solar atmosphere was investigated in this study (based on the limited Sunrise/IMaX FOV), so that a possible bias cannot be ruled out.

\subsubsection{Magnetic carpet}

The dynamics of the QS is quite complex since the atmosphere above the magnetic network is permanently and everywhere filled with small-scale magnetic flux in the form of magnetic loops emerging from below. This small-scale magnetic mesh is usually referred to as "magnetic carpet" (Title and Schrijver 1998, and see Fig. 21). The structure of this mixed-polarity network is driven by processes such as magnetic flux emergence, flux cancelation as well the coalescence and fragmentation of magnetic elements (Parnell 2001). Widely used for the statistical analysis of the properties of magnetic features associated with the magnetic carpet are potential and LFF field models (see Sect. 2.2.1). Both methods are based on the observed LOS photospheric magnetic field and deliver similar results concerning the length of field lines, the height of their apex in the atmosphere and the approximate field strength (Wiegelmann et al. 2010a).

Therefore, although the evidence for a common canopy-like field (nearly horizontal field overlying a nearly field-free region) is relatively strong, the criticisms of the canopy as a boundary between open and closed field lines as well as between high and low-beta plasma are better found. Therefore, such views of the canopy do need revising. 


\subsubsection{Role of magnetic fields in coronal and chromospheric heating}

The fact that the plasma temperature in the solar corona is a factor of $\approx 10^{3}$ higher than in the photosphere motivates the search for plausible mechanisms yielding the needed amount of energy involved. Throughout the last decade, a number of authors, including Walsh and Ireland (2003), Aschwanden (2005), McIntosh et al. (2011), Wedemeyer-Böhm et al. (2012), Winebarger et al. (2013), demonstrated that one of the several mechanisms, involving small-scale X-ray jets, bright points, micro- and nanoflares as well as Alfvén and MHD turbulence and waves, are able to provide partial explanations for the extreme heating of coronal plasma. Mass and energytransport between the lower atmosphere (photosphere and chromosphere) and the outer atmosphere (corona) is, in general, a challenging concern. Not only because of the complicated and highly dynamic structure of these layers, but also because of the highly non-linear physical processes involved. Nevertheless, some progress was made already during the Skylab-era (see Withbroe and Noyes 1977, for a review). The discussion of coronal and chromospheric heating is well outside the scope of the present paper, however, and we therefore refer the interested reader to the specialized review by Klimchuk (2006).

Numerical experiments, carried out by Schrijver and Title (2002) showed that lowlying chromospheric fields are well outlined by the magnetic connection between magnetic elements observable, e.g., in the form of $\mathrm{H} \alpha$ fibrils. Although a complex magnetic field structure was found, which contained multiple null points representing a potential source for reconnection, they were found to reside in the low atmosphere only and thus were not likely to significantly contribute to coronal heating. Régnier et al. (2008) used a potential magnetic field model based on Hinode/SOT-SP magnetograms to investigate the relation of coronal and photospheric null points and confirmed a shortage at coronal heights. In particular, they found that $98 \%$ of the null points present in the model were located at photospheric or chromospheric heights, and only $2 \%$ were found to be situated at coronal heights (see Fig. 22). Thus, they confirmed earlier findings based on numerical experiments which stated that magnetic reconnection at coronal null points is unlikely to be the source for coronal heating.

Wiegelmann et al. (2013) investigated a 22-minute time series of potential field equilibria extrapolated from Sunrise/IMaX data and found that the magnetic connectivity in the upper solar atmosphere changes rapidly, with a recycling time of $\approx 3 \mathrm{~min}-$ a short time compared to $\approx 14 \mathrm{~min}$ in the photosphere. An estimation of the upper limit for the free magnetic energy, which might be converted to heat by magnetic reconnection was still somewhat too small to be the dominant source for chromospheric and coronal heating in the QS. The same conclusion was reached by Chitta et al. (2014), based on a magneto-resistive computation starting from series of SDO/HMI and from Sunrise/IMAX magnetograms.

\subsubsection{Force-freeness of quiet-Sun magnetic fields}

Going beyond the potential field approach and applying force-free extrapolation methods (see Sect. 2.2), to picture the magnetic field in the outer solar atmosphere above quiet-Sun regions, are tempting. This is, however, challenging, since force-free mod- 
Fig. 22 Spacial distribution of the projected location of magnetic null points, computed from a potential field model. Blue, yellow and red circles mark the locations of photospheric, chromospheric, and coronal nulls, respectively. The quantity of coronal nulls is outnumbered by that of the photospheric and chromospheric nulls. Photospheric magnetic field in a quiet-Sun area observed with Hinode/SOT on 24 June 2007 at 22:09 UT. The gray-scale background depicts the LOS magnetic field component, in the range $\pm 50 \mathrm{G}$. Black/white represents negative/positive polarity. (Figure 1 of Régnier et al. 2008. Reproduced with permission, (C) ESO)

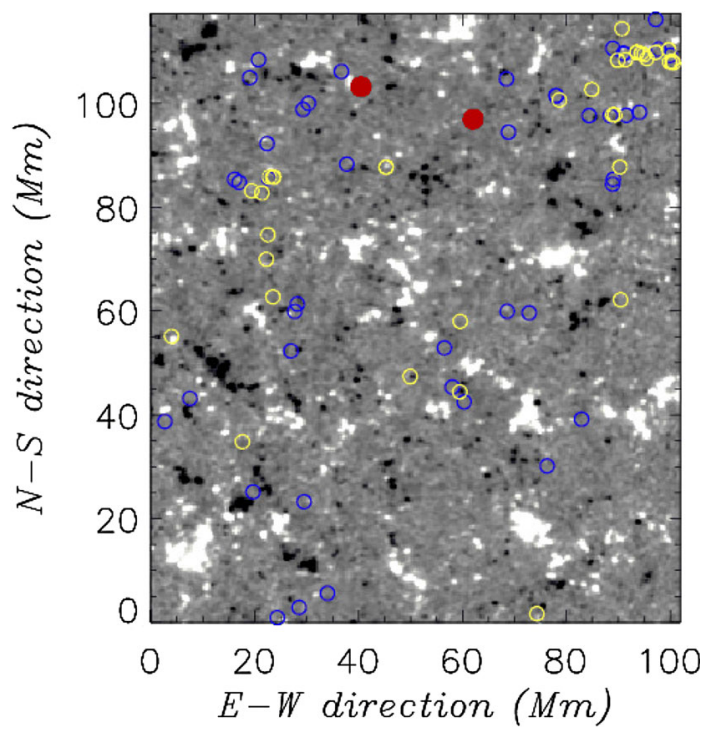

els are based on the magnetic field measurements at low atmospheric heights, i.e., routinely at photospheric, or occasionally at low chromospheric heights. Additionally, in the QS one has to deal with a poor signal-to-noise ratio of the transverse magnetic field measurements. Nevertheless, Zhao et al. (2009) aimed to compare the measured horizontal field of Hinode/SOT-SP vector magnetograms with the horizontal field component of an associated potential field model. They confirmed that the quiet Sun's magnetic field is non-potential, that it carries significant electric currents, and that it is sheared, on average by about $40^{\circ}$ with respect to a potential field. A different approach is to employ the magneto-resistive method of van Ballegooijen, which returns force-free fields without the need of making use of the measurements of horizontal photospheric magnetic field components. This approach has been taken by Chitta et al. (2014).

The difficulty in modeling QS-related coronal magnetic fields using different NLFF methods has recently been demonstrated by Liu et al. (2011b): the results gained from the different modeling techniques deviated considerably. The problems may run deeper, since even the assumption that the magnetic field in the QS is force-free is questionable. Following, Schrijver and van Ballegooijen (2005) it is unlikely that the quiet Sun's coronal magnetic field is force-free or even quasi-steady. One of the necessary conditions a force-free environment has to meet is that, at a given height, the surface integral $\int_{S} B_{\text {vertical }}^{2}-B_{\text {horizontal }}^{2} \mathrm{~d} S$ vanishes (see Aly 1989, for details). This condition cannot be fulfilled if, on average, the (unsigned) horizontal field is much larger than the vertical one.

This means that a predominantly horizontal field cannot exist at the base of a forcefree environment, and if the horizontal flux is on average about a factor of five higher (as claimed by Lites et al. 2008) the quiet-Sun photosphere is even farther away from a force-free state than photospheric active-region fields are. It should be mentioned 


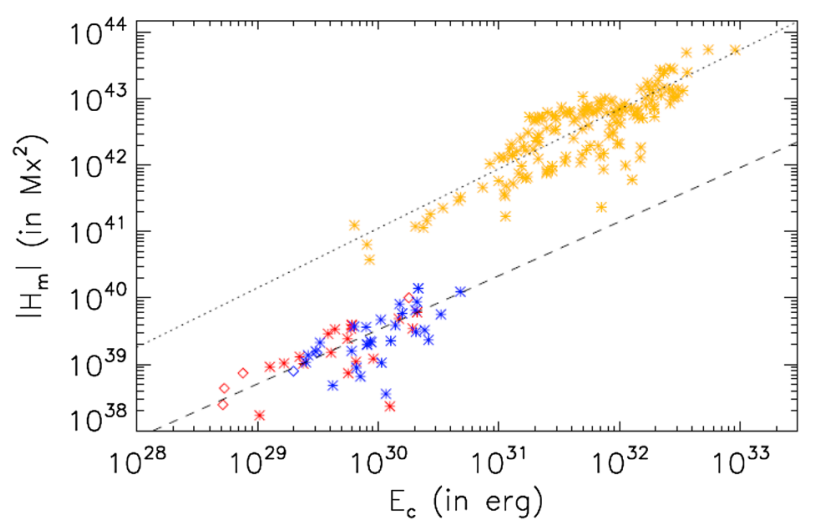

Fig. 23 Free magnetic energy ( $E_{c}$; horizontal axis) in relation to the magnitude of the relative magnetic helicity ( $H_{m} ; y$-axis). The yellow stars in the upper right part of the graph correspond to the values calculated for a number of ARs by Tziotziou et al. (2012). Blue/red plot symbols correspond to a positive/negative total helicity budget of solar quiet regions, respectively. Asterisks and diamonds mark regions centered within $\pm 30^{\circ}$ heliographic latitude and higher latitudes than that, respectively. The dashed and dotted lines represent least-squares fits to the values calculated for the quiet-Sun and active-region areas, respectively. (Figure 6 of Tziotziou et al. 2014. Reproduced with permission (C) ESO)

though, that Danilovic et al. (2010b) found that a ratio closer to 1 is also compatible with observations. Obviously, Aly's condition is not fulfilled in the internetwork photosphere where the horizontal field is on average much stronger than the vertical field, which has important implications for the reliability of force-free magnetic field reconstructions in the QS. The fact that these fields are definitely far from being force-free in the photosphere and that most of the weak (nearly) horizontal fields are the tops of very short and low-lying loops (Martínez González et al. 2007; Centeno et al. 2007; Martínez González and Bellot Rubio 2009; Danilovic et al. 2010a) which do not reach up to layers where the field is closer to being force-free, the impact of the plasma on the field cannot be neglected.

\subsubsection{Magnetic energy and helicity budget}

Despite the difficulties and limitations of applying force-free models to quiet-Sun areas (see Sect. 5.2.5), NLFF models have recently been applied to investigate the magnetic energy and helicity budget in the QS. Tziotziou et al. (2014) performed a statistical study with a large number of quiet-Sun vector magnetograms from Hinode/SOT-SP. They found that the QS has no dominant sense of helicity (in contrast to ARs) and that both the free magnetic energy and the relative magnetic helicity are correlated with the area occupied with network elements. Consequently, the free magnetic energy and the relative helicity are correlated as well (see blue/red stars and diamonds in Fig. 23, respectively). In an earlier work, Tziotziou et al. (2012) investigated these quantities for ARs (shown as yellow stars in Fig. 23, for comparison). Both energy and helicity are much lower in the QS, compared to ARs, which is naturally caused by the lower magnetic flux they contain. The authors also noted that the investigated quiet-Sun areas are closer to a potential field than the ARs, which is another reason for a lower 
free energy in the QS. See also Sects. 3.4 and 4.6 for magnetic helicity investigations on global scales and in ARs, respectively.

\subsection{Small-scale dynamics}

The observations of the coronal magnetic field show numerous dynamic phenomena on small as well as larger (active region) scales. In particular, we now know that the term "quiet Sun" is not really adequate to describe the coronal field even outside of ARs, since numerous dynamic phenomena on small scales are known to occur there (Schadee et al. 1983; Parker 1988; Shibata et al. 1992).

Chesny et al. (2013) found the first evidence for sigmoidal (i.e., S shaped) loops in the QS using SDO/AIA data. These were interpreted as a clear indicator for nonpotential magnetic fields. So far S-shaped loops had been observed to connect within ARs or to connect ARs on either side of the solar equator (i.e., in the form of TELs) only. S-shaped loops in the QS indicate that similar dynamic phenomena as in ARs might occur here, but on smaller scales. In fact, events similar to small versions of flares and CMEs are found in the QS (Innes et al. 2009, 2010), which mostly attract less attention than their impressive, and sometimes globally disturbing, active-region counterparts. In fact, outside of ARs the Sun displays a broad range of smaller-scale, transient phenomena which involve only a fraction of the energy that is involved in the dynamics associated to the predominant large-scale activity sites.

\subsubsection{Jets}

Already in the 80s of the last century, Brueckner and Bartoe (1983) reported observations of high-energy supersonic jets in quiet-Sun areas. They found that the associated shock waves reached to heights of about 4 to $16 \mathrm{Mm}$. The authors applied a cloud model for the solar wind and assumed that the entire kinetic energy of the wind is provided at the Sun's surface. This involves the presence of emerging flux and a field strength of $15 \mathrm{G}$ and a flux emergence of $1.3 \times 10^{15} \mathrm{Mx}$ is required to power the corresponding high speed jets. In their opinion, magnetic forces are the most likely cause for the jets and a correlation to active areas in the QS was found. Moreover, the influence of the individual jets was discernible as fine structures in the solar wind flow at a distance of one AU. Later, X-ray jets were observed by Shibata et al. (1992, 1994), although not necessarily in quiet-Sun regions.

Yokoyama and Shibata (1995) carried out 2D resistive MHD simulations including a simplified convection zone, photosphere, chromosphere and corona. They found that magnetic reconnection plays an important role for activity in the upper solar atmosphere and can be a common cause of both hot X-ray jets, as well as cooler loop brightening that become visible in $\mathrm{H} \alpha$. In the former case, plasma is heated up to temperatures between 4 and $10 \mathrm{MK}$ and is accelerated to the Alfvén speed of about $100 \mathrm{~km} \mathrm{~s}^{-1}$. In the latter case, cool $\left(\approx 10^{4} \mathrm{~K}\right)$ chromospheric material is accelerated almost without plasma heating. The authors concluded that the combined energy of cool-loop brightening and hot-jet acceleration might be significantly larger than previ- 
Fig. 24 Schematic view on the reconnection occurring in the solar corona upon emerging magnetic flux from the center of a supergranule below with a pre-existing magnetic field. Near the reconnection $\mathrm{X}$-point, the temperature is enhanced and outflows are observed presumably as EUV/X-ray jets in the corona and $\mathrm{H} \alpha / \mathrm{Ca}$ surges in the chromosphere. (Adapted from Figure 10 of Jiang et al. 2012. (C) AAS. Reproduced with permission)

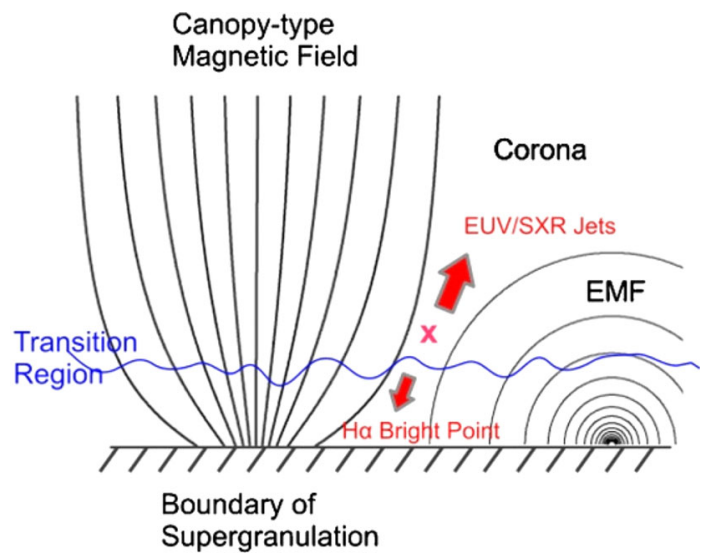

ously thought. Also the energy in loop brightenings might have been underestimated from previous observations.

The role of magnetic reconnection for heating and dynamics of the solar corona was revisited by Shibata et al. (2007), this time observationally with Ca II H data from Hinode/SOT. So-called "anemone jets" were found both in the corona and in the chromosphere. The authors interpreted the frequently observed small chromospheric anemone jets as a manifestation of ubiquitous magnetic reconnection. The coexistence of cooler chromospheric material, that becomes visible in $\mathrm{H} \alpha$ and the hot jets expected from numerical experiments by Yokoyama and Shibata (1995) was observationally confirmed. The combined energy of these events was more than two orders of magnitude too small to explain the heating the active corona. Nonetheless, the authors pointed out that the jets observed by Hinode/SOT are only the largest events and a great number of smaller jets might have gone undetected, but may also contribute to the heating.

Recently Jiang et al. (2012) presented 2.5D MHD simulations of the processes involved in canopy-type magnetic configurations triggered by newly emerging magnetic flux into a pre-existing field configuration at the boundary of a supergranule. They found hot $\left(\approx 10^{6} \mathrm{~K}\right)$ and cold $\left(\approx 10^{4} \mathrm{~K}\right)$ jets originating from coronal microflares and associated them to what is usually observed in coronal and chromospheric images as EUV or HXR jets and $\mathrm{H} \alpha / \mathrm{Ca}$ surges, respectively (Fig. 24). This magnetic reconnection between newly emerging magnetic flux and the ambient field plays a major role for jets has been confirmed in numerical experiments recently by Moreno-Insertis and Galsgaard (2013). For a recent review on small-scale, jet-like events at chromospheric levels, we refer to Tsiropoula et al. (2012), as well as to Sect. 6.2.4 for polar jets.

\section{Coronal holes}

CHs are regions of strongly reduced emissivity at coronal temperatures and, consequently seen as dark features in coronal images (which is why they are called "holes"; see Fig. 25). The magnetic field of CHs is mainly open, i.e., organized in magnetic 


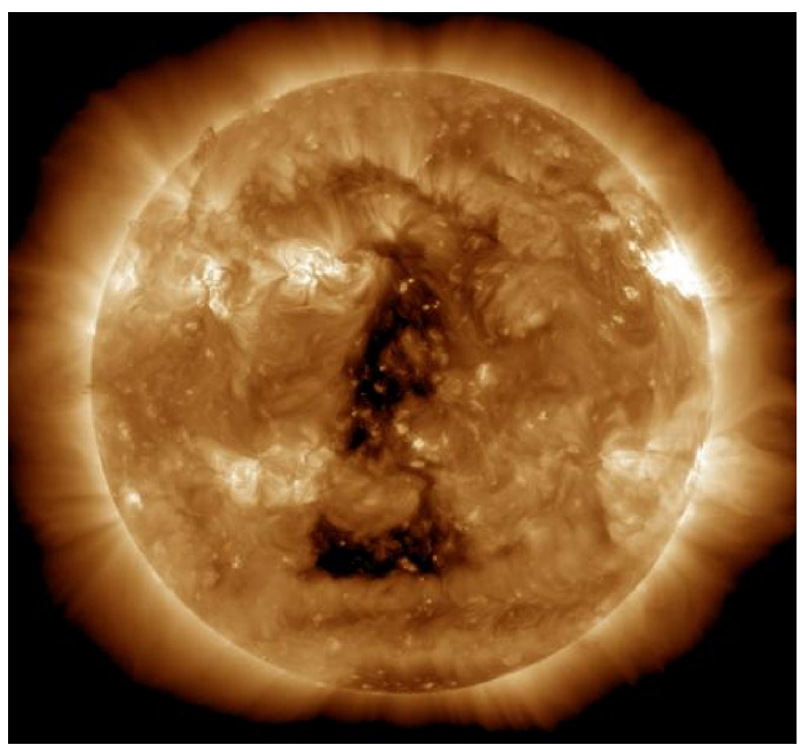

Fig. 25 A large coronal hole observed with SDO/AIA at $193 \AA$ on 13 March 2012. It can easily be distinguished from its quiet Sun surrounding as the region of lowest EUV emission, while ARs exhibit strongest emission. Source http://phys.org/news/2012-03-huge-coronal-hole-solar.html

funnels having their footpoints in the network. Along open fields, charged particles can escape the outer solar atmosphere, leaving behind plasma of strongly reduced density and slightly reduce temperature, if compared to the quiet Sun's corona. The main motivation for the research of CHs since the 1970s was to understand the role of CHs in terms of the mass and energy flow between the Sun's surface and the heliosphere. For a recent overview we refer to Cranmer (2009). One distinguishes between polar CHs (PCHs) and equatorial CHs (ECHs; see Del Zanna and Bromage 1999).

For techniques used to analyze observations of $\mathrm{CHs}$, it makes a difference whether the holes are observed on the disk or above the solar limb. Discussing the corresponding challenges and issues, however, lies outside the scope of this work and we refer to the review by Cranmer (2009). Here, we focus on the magnetic field structure in CHs. As other magnetic structures on the Sun, $\mathrm{CHs}$ and the corresponding solar wind streams change over the solar cycle (Harvey and Sheeley 1979). For changes of the magnetic structure of CHs in the course of the solar cycle see Sect. 3.3.

\subsection{Properties of coronal holes}

\subsubsection{Properties of photospheric fields associated with coronal holes}

Almost continuous observations of $\mathrm{CHs}$ and measurements of the associated photospheric magnetic fields are available since the Skylab era in the 1970s. To our knowledge, a first review on observations of $\mathrm{CHs}$ and the underlying solar magnetic field was presented by Harvey and Sheeley (1979). Somewhat larger CH-averaged values, rang- 
ing from 0.8 to $17 \mathrm{G}$, with an average value of nearly $8 \mathrm{G}$, were found by Wiegelmann and Solanki (2004). Conclusions on the magnetic structure of $\mathrm{CHs}$ were at that time mainly based on the photospheric LOS magnetic field observations (e.g., from KPNO). Unfortunately, at that time, the magnetic field data were subject to major uncertainties owing to systematic errors (a factor of $\gtrsim 2$ ). Nevertheless, it already became clear that the photospheric field in which $\mathrm{CHs}$ are rooted is dominated by fields of a single polarity, i.e., either positive or negative. In ECHs, for instance, the main polarity of the holes dominated in the range of 58 to $95 \%$ and, on average, $77 \pm 14 \%$ (Wiegelmann and Solanki 2004). Equatorial quiet-Sun regions were approximately flux balanced in the range of 2 to $29 \%$ and, on average, $9 \pm 9 \%$. The magnetic field strength of these photospheric fields, averaged over the whole $\mathrm{CH}$, was found to lie in the range 0.5 to $7 \mathrm{G}$ (Harvey and Sheeley 1979, and references therein). It is noteworthy that there is some scatter in the values found, depending on the observations used for analysis.

As in ARs and in the QS, reliable magnetic field measurements are mainly available at photospheric levels. Because of the poor signal-to-noise ratio in weak-field regions, it is difficult to measure the weak transverse field with high accuracy. As a consequence, observational studies and coronal magnetic field models are often based on LOS magnetic field measurements. An additional problem arises for the observation of $\mathrm{CHs}$ in polar regions, where foreshortening effects cannot be neglected (since the spatial resolution in the direction to the limb decreases) and where limb darkening can also become an issue.

\subsubsection{Modeling of coronal-hole magnetic fields}

Similar to the QS and global magnetic fields, potential field models based on photospheric field measurements are used as a first approximation of the magnetic field in CHs. Note, however, that also when applied to model the magnetic field in coronalhole areas potential field models have their limitations (see Sect. 5.2.5 for details). The magnetic field structure of CHs is often also studied with the help of PFSS models (see Sects. 2.2.1 and 3.1). In their output, coronal-hole areas are easily identified by their open magnetic fields (i.e., field lines connecting the photosphere with the source surface). A powerful tool for $\mathrm{CH}$-investigations is the combination of flux-transport models in the photosphere and coronal field modeling with PFSS (see Sect. 2.3.3 for a description of the method and Sect. 6.3.1 for an application to ECHs).

Wang (2009) pointed out that coronal-hole areas can be reasonably well reproduced over the entire solar cycle when assuming the source surface of a PFSS model to be located at a constant height of 2.5 solar radii (i.e., $\approx 1.74 \times 10^{9} \mathrm{~m}$ ) above the photosphere. The main contribution to the open flux in PFSS models arises from the dipole and quadrupole moment of the Sun's global magnetic field-the latter, especially around solar maximum. Higher-order multipoles decrease too rapidly with height to contribute significantly at the source surface. Note that taking the quadrupolar field into account does not substantially change the resulting rotational properties of the coronal-hole associated magnetic field: the photospheric field (represented by the highorder multipole moments) rotates differently while the coronal field (characterized by the low-order multipoles) rotates almost rigidly. 


\subsection{Polar coronal holes}

During solar activity minimum, large $\mathrm{CHs}$ occur in the polar regions as a consequence of the dominating dipolar moment of the Sun. One has to keep in mind, however, that all measurements concerning near-polar areas, are carried out from the ecliptic, i.e., from a highly inclined viewpoint with respect to the target region. With the launch of the Solar Orbiter mission, scheduled for 2017, this drawback will finally be overcome.

\subsubsection{Magnetic flux in polar coronal holes}

Hinode/SOT-SP provided the capability of studying the full photospheric magneticfield vector around the Sun's south polar region (Tsuneta et al. 2008a). Unipolar, vertical flux tubes with $\mathrm{kG}$ fields, scattered all over the $\mathrm{PCH}$ (ranging from $70^{\circ}$ to $90^{\circ}$ solar latitude) and ubiquitous horizontal fields were found. The strong $(\mathrm{kG})$ vertical spots are unipolar, while the average field strength of the entire FOV is only about $10 \mathrm{G}$, depending on the filling factor. In total, Tsuneta et al. (2008a) found the horizontal magnetic flux in the polar region, around solar minimum, to be almost twice the vertical flux, namely $4.0 \times 10^{21}$ and $2.2 \times 10^{21} \mathrm{Mx}$, respectively.

Jin and Wang (2011) revisited Hinode/SOT-SP vector magnetic field measurements in a PCH and compared it to measurements of quiet-Sun areas close to the solar limb and the disk center, as well as with low-latitude CHs. They also uncovered an imbalance of vertical and horizontal field in the polar region, given an average vertical and horizontal flux density of $\approx 100 \mathrm{G}$ and $\approx 1 \mathrm{kG}$, respectively. They estimated that such patches of strong vertical field occupy $\approx 7 \%$ of the $\mathrm{CH}$ area investigated in their study. The authors also revealed a significant amount of magnetic flux with a polarity opposite to the dominant polarity. This implied that only about one-third of the magnetic flux in the analyzed polar region could be assigned to be actually open magnetic flux. They also found that the ratio of dominant to minor flux for low-latitude and PCHs is similar, but that the total vertical magnetic field strength in polar holes is about $60 \%$ higher.

In general, we expect that as an increasingly larger fraction of the magnetic flux within $\mathrm{CHs}$ is detected, the percentage of open flux relative to the total flux in a $\mathrm{CH}$ will continue to decrease. However, we also expect that much of the hidden flux is weak and ordered on very small spatial scales. Consequently, much of this flux is expected to be restricted to very low-lying loops that do not reach into the corona, as found by Wiegelmann et al. (2010a) in the quiet Sun.

\subsubsection{Comparison to the quiet-Sun magnetic field}

Using Hinode/SOT measurements, Ito et al. (2010) compared a polar $\mathrm{CH}$ region with a quiet-Sun region near the East limb at an epoch close to solar activity minimum (Fig. 26). The total magnetic flux and area covered by kG-fields was found to be larger in the north-polar region than in the QS near the East limb. Also, while the vertical magnetic field was found to be nearly balanced in the considered quiet Sun, near-limb area, one polarity clearly dominated the polar regions. In particular, around $90 \%$ of the features with a magnetic field magnitude of more than $500 \mathrm{G}$ were of the same 


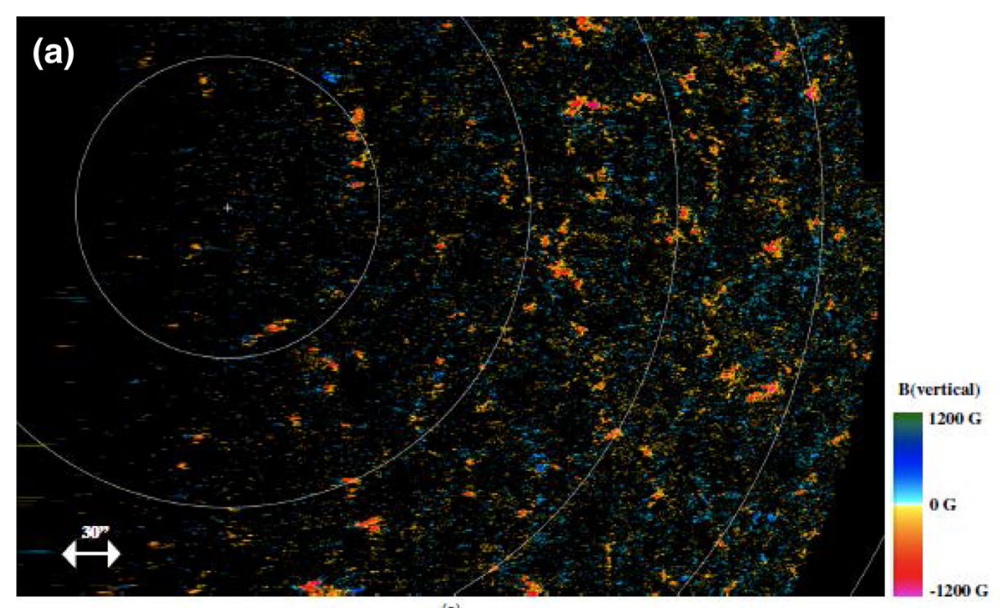

(a)

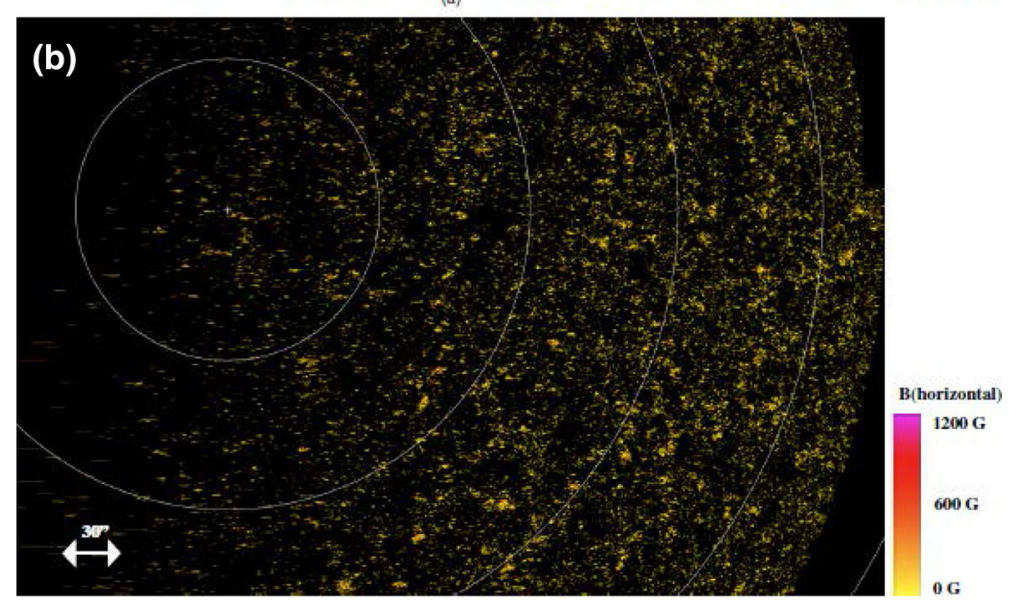

Fig. 26 Maps of the signed strength of the a vertical and $\mathbf{b}$ horizontal components of the magnetic field vector on 25 September 2007, converted to a view from above the north pole of the Sun. East/west is to the left/right. The pixel size is 0.' 16. Black areas mark locations where the magnetic field strength has not been obtained, because the associated polarization signal did not exceed a given threshold above the noise level. The color code in a represents the signed strength of the vertical magnetic field, with red/blue representing negative/positive polarity. Many of the horizontal field concentrations in $\mathbf{b}$ are co-located with the vertical field patches in a. (Figure 4 from Ito et al. 2010. (C) AAS. Reproduced with permission)

polarity. A PFSS model revealed mainly open magnetic fields in the north-polar $\mathrm{CH}$ and predominantly closed structures in a quiet-Sun region near the East limb (see (a) and (b) in Fig. 27, respectively). Because of one dominating polarity in the hole, the opposite flux cancels out by low-lying loops and above a certain height all field lines are open. Note that this is a general property of $\mathrm{CHs}$ which has also been found for ECHs (see Sect. 6.3.2). The authors also found that the horizontal fields are similar in the QS and at the poles and interpreted this as evidence for a local dynamo. A global dynamo would create different fields in polar and equatorial regions, they argued; see also Sect. 1.1.3. 


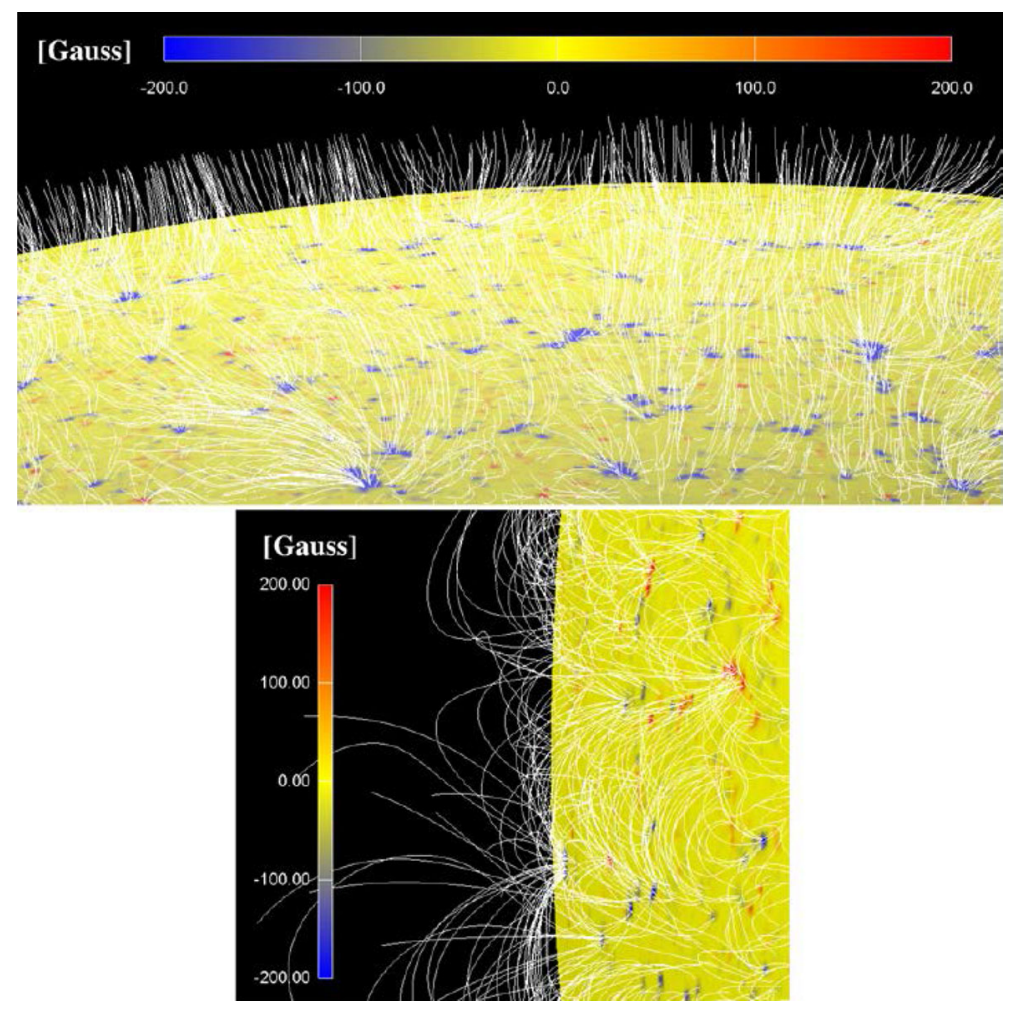

Fig. 27 Selected field lines, calculated from a PFSS model, outlining the coronal magnetic field structure associated to a $\mathrm{CH}$ a around the north pole of the Sun and $\mathbf{b}$ in a quiet-Sun area near the East limb. It can be seen that the majority of field lines that originate in $\mathrm{kG}$-features fan out just above the photosphere (in a canopy-like fashion) and that they are open. The color-coded surface indicates the vertical magnetic field, where blue/red represents negative/positive polarity. It is evident that the polar region is dominated by a specific (negative, in this case) polarity, while the QS represents an environment of mixed polarity. (Figure 11 from Ito et al. 2010. (C) AAS. Reproduced with permission)

\subsubsection{Polar plumes}

Polar plumes are visible in the form of thin streamers above the solar limb in the polar regions. They have been studied intensively, e.g., by a coordinated observing campaign ( $\mathrm{SOHO}$ and several of other space-borne and ground-based instruments; see DeForest et al. 1997). A comprehensive review was given by Wilhelm et al. (2011), including discussion of the 3D structure of polar plumes, details of their generation and of their interaction with the solar wind. Here, we concentrate on the magnetic field structure of polar plumes.

Supported by potential field models, polar plumes are associated to open fields on large scales (Suess 1982). While the source region of these plumes is usually not visible in coronal EUV images, stereoscopic reconstructions by Feng et al. (2009) showed that they connected to photospheric magnetic patches. Wang and Sheeley (1995) and Wang (1998) proposed that plumes are produced by the reconnection of emerging mixed- 
polarity fields with previously existing unipolar fields. Raouafi et al. (2008) reported that most of the studied coronal jets in polar regions are generally followed by plumes. The authors pointed out the common feature shared by plumes and jets: a mixedpolarity field area where their footpoints are located. In their picture, flux emergence and subsequent opening of previously closed loops by magnetic reconnection provide the energy for jet acceleration and eventually produce the open (plume) field geometry. For $70 \%$ of the observed polar jets, a plume was found to form within minutes to hours after the appearance of the jet. Such an association is plausible, given that the formation mechanism of jets is also thought to be the reconnection of emerging flux with previously present unipolar field (e.g., Yokoyama and Shibata 1995; Canfield et al. 1996).

\subsubsection{Polar jets}

Jets are ubiquitous dynamical features which occur in CHs, ARs and the QS. (For a discussion of jets occurring in the QS, see Sect. 5.3.1.) For example, Cirtain et al. (2007) detected about 10 fast jets per hour in a PCH. Detailed analysis revealed that at least a subset of jets is characterized by two distinct velocities: one close to the sound speed $\left(\approx 200 \mathrm{~km} \mathrm{~s}^{-1}\right)$ and another one in the order of the Alfvén speed $\left(\approx 1,000 \mathrm{~km} \mathrm{~s}^{-1}\right)$. This might be explained with the following scenario. Initially, the outflow triggered by reconnection has a velocity close to the Alfvén speed. Due to the transit of a reconnected magnetic field line to a relaxed configuration, Alfvén waves become excited, which might also contribute to the acceleration of the fast solar wind. The reconnection process also has the effect of heating the coronal plasma by converting magnetic to thermal energy. As a consequence, the plasma expands, which also leads to a plasma outflow. Its speed, however, is significantly lower (sound speed) than the outflow component caused by magnetic reconnection.

Using spectroscopic data from SOHO/SUMER and Hinode/EIS, Kamio et al. (2009) investigated the formation of jets in the transition region and coronal environment of CHs. They deduced that the open magnetic fields associated with the jets were rooted in $\mathrm{kG}$ vertical fields at photospheric levels. They also found that both explosive phenomena and cool up-flows were caused by magnetic reconnection with low-lying loops in the transition region, confirming and extending earlier results. Yang et al. (2011b) investigated the boundaries of the equatorial extension of a PCH using co-observations of the photospheric magnetic field from SDO/HMI and the coronal plasma EUV emission with SDO/AIA. A number of jets were recognized in the EUV images and interpreted as signatures of magnetic reconnection. The latter was supported by co-temporal emergence and cancelation of magnetic flux seen in the photospheric magnetic field data. The coexistence of open and closed fields at $\mathrm{CH}$ boundaries naturally results in energy deposition by multiple reconnection events, those thought to produce jets.

\subsubsection{Contribution to the solar wind}

Tian et al. (2011) observed high-speed outflows of $\approx 120 \mathrm{~km} \mathrm{~s}^{-1}$ in plume-like structures in polar and ECHs, as well as quiet-Sun regions in SDO/AIA images. Comparison with a potential coronal magnetic field model based on SDO/HMI measurements 
led the authors to conclude that plume-related jets do not necessarily contribute to the solar wind. This confirmed what was found earlier by Feng et al. (2009), using STEREO/SECCHI data, namely that the contribution of polar plumes to the fast solar wind is insignificant. Note, however, that studies of outflows in polar plumes and inter-plume-regions are contradictory. For instance, Gabriel et al. (2003) claimed that about half of the fast solar wind at a distance of 1.1 solar radii originates from plumes. A corresponding discussion is beyond the scope of this work and we refer to section 4 of Wilhelm et al. (2011) for a detailed review of the relevant literature.

\subsection{Equatorial coronal holes}

During cycle phases of higher solar activity, CHs are present at all latitudes. ECHs are smaller and persist for a shorter time than PCHs. Nevertheless, they persist over several solar rotations. Many low-latitude holes are located close to the edges of ARs. This helps to understand the changes of $\mathrm{CHs}$ in the course of the solar cycle: because ARs tend to emerge closer and closer to the solar equator, $\mathrm{CHs}$ exhibit a similar trend. In some cases, ARs appear even within these CHs (see section 3 in Cranmer 2009, for details). As a consequence of the emerging $\mathrm{AR}$, the $\mathrm{CH}$ decreases in size.

\subsubsection{Formation and evolution}

In contrast to the differential character of the solar rotation in the sub-surface layers of the Sun, ECHs at coronal heights rotate more rigidly (Timothy et al. 1975), but still with a differential character (Insley et al. 1995). Glencross (1974) suggested "magnetic merging", or more precisely magnetic reconnection, of oppositely directed magnetic arches could possibly form $\mathrm{CHs}$ and also explain their more rigid rotation.

Associated numerical experiments have been carried out by Wang et al. (1996) and as a possible origin of ECHs, the interaction of bipolar ARs has been identified, which is able to explain the imbalance of magnetic flux that is characteristic for CHs. Modeling a bipolar magnetic region superposed on an axis symmetric dipole global field, Wang et al. (1996) found that both, the local photospheric field as well as the overlying global coronal field, determine the location of open field regions. The rotation of the $\mathrm{CH}$, however, is then controlled by the bipolar magnetic region with which it co-rotates (see Fig. 28). During this process, the coronal magnetic field has to change its topology by magnetic reconnection from closed to open, and vice versa when entering and leaving the $\mathrm{CH}$. By definition, magnetic reconnection is prohibited in potential field models, as is the breaking down of the frozen-in condition of the high-conductivity coronal plasma.

Therefore, physically more advanced approaches, able to investigate the nature of the magnetic reconnection involved in the development of $\mathrm{CHs}$, were carried out by Lionello et al. (2005). They found that while the magnetic structure of a $\mathrm{CH}$ might be relatively stable, magnetic flux may permanently move in or out of the region covered by the $\mathrm{CH}$, causing reconnection to happen. The transitions from closed to open magnetic fields at the boundaries of $\mathrm{CHs}$ were suspected to be the origin of the slow (with velocities of $\lesssim 450 \mathrm{~km} \mathrm{~s}^{-1}$ ) solar wind (Wang et al. 1996). Interchanging 

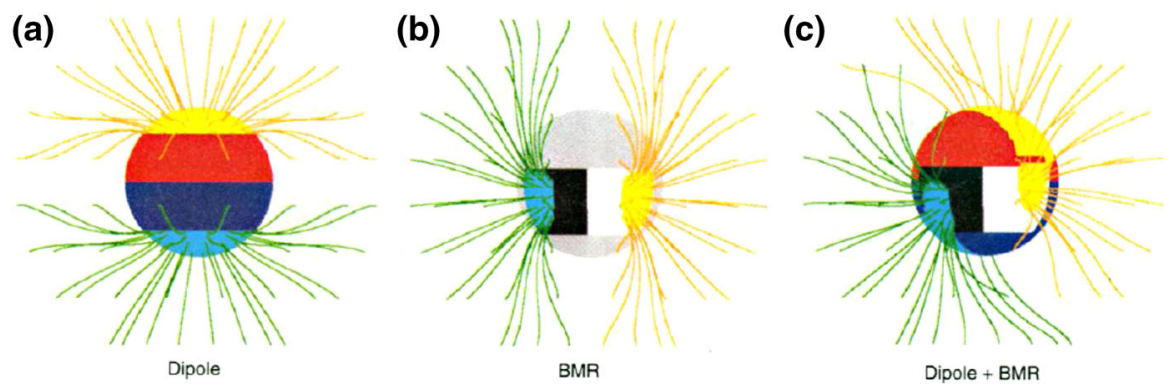

Fig. 28 Examples for idealized open magnetic field regions: Panel a shows an axisymmetric dipole, panel b a bipolar magnetic region (BMR) and panel c a superposition of dipole and BMR. Orange and green field lines originate in positive and negative flux regions, respectively. The color coding on the solar surface corresponds to different regions: white and black to positive and negative polarities in ARs, yellow and light blue to positive and negative polarities in $\mathrm{CHs}$, red and dark blue to positive and negative background fields. (Adapted from Figure 4 of Wang et al. 1996. Reprinted with permission from AAAS)

reconnection between open and closed fields at the boundary of $\mathrm{CHs}$ is thought to be responsible for, or at least related to coronal jets and polar plumes.

Recently, Petrie and Haislmaier (2013) used synoptic magnetograms from GONG to approximate the corona with a potential-field model and compared it to synoptic EUV maps from STEREO. They investigated the relationship between decaying ARs and $\mathrm{CHs}$ at low latitudes. Newly emerging ARs generally result in significant changes of the global coronal magnetic field structure and cause the re-shaping of the streamer belt. Decaying ARs which were found to evolve steadily and gradually, however, would not yield a considerable change of the global field, even though they left behind a considerable flux imbalance. The authors argued that some of the imbalanced flux, nevertheless needs to (re)connect elsewhere and thus to form the streamer belt or the open fields.

\subsubsection{Comparison with quiet-Sun magnetic fields}

The local, small-scale magnetic field structure of ECHs in the upper solar atmosphere has been investigated by Wiegelmann and Solanki (2004) under the presumption that electromagnetic radiation from coronal plasma mainly originates from closed magnetic loops, while open field lines remain almost invisible owing to their strongly reduced plasma density. A statistical study, using a potential field model based on SOHO/MDI magnetograms, revealed that only small closed magnetic loops exist within the $\mathrm{CH}$. The apex of these loops does not reach coronal heights. Above a certain height, all field lines were found to be open since the minority flux has been canceled out. In contrast, quiet-Sun areas are more or less flux balanced and thus contain field lines of various apex heights, including numerous field lines reaching up into the corona (see Fig. 29). For corresponding investigations of PCHs see Sect. 6.2.2 and Fig. 27. Yang et al. (2011a) compared the magnetic field vector field in two ECHs and the QS using Hinode/SOT-SP data. They found that horizontal magnetic fields, inclination angels as well as the current density and current helicity are stronger in $\mathrm{CHs}$ than in the QS. The authors also concluded that the magnetic field in both QS and $\mathrm{CH}$ is non-potential (see also Sect. 5.2.5). 

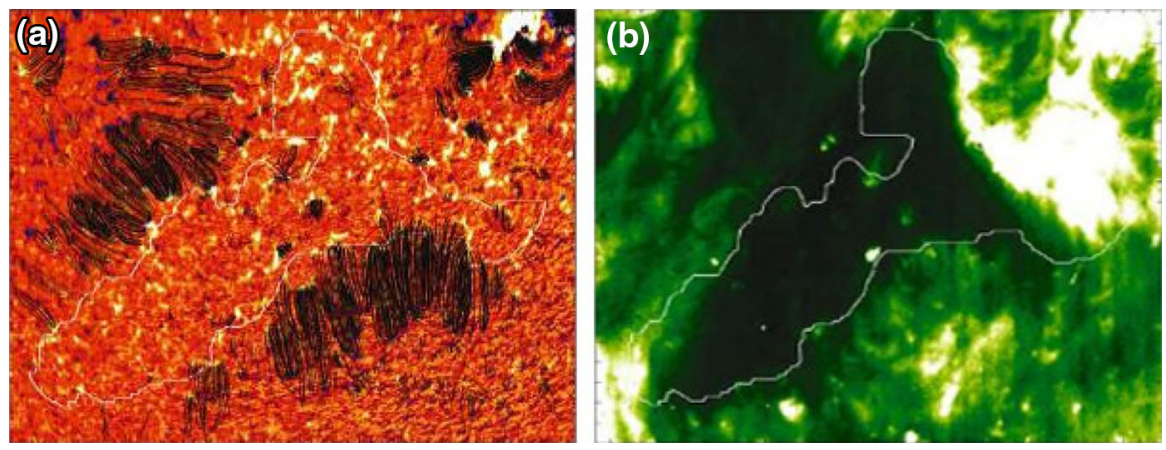

Fig. 29 a SOHO/MDI magnetogram and overlaid closed magnetic field lines (black) obtained from a potential field model. Only closed field lines with a field strength of $\geq 20 \mathrm{G}$ are shown. One can see that outside of the $\mathrm{CH}$ boundary, the number of closed loops is significantly higher and that these loops are also longer. b Co-spatial EUV image from SOHO/EIT at $195 \AA$ A. The boundary of an ECH is outlined by a white contour in both images. The $\mathrm{CH}$ area is seen as a region of reduced emissivity. (Adapted from Figure 3 of Wiegelmann and Solanki 2004. With kind permission from Springer Science and Business Media)

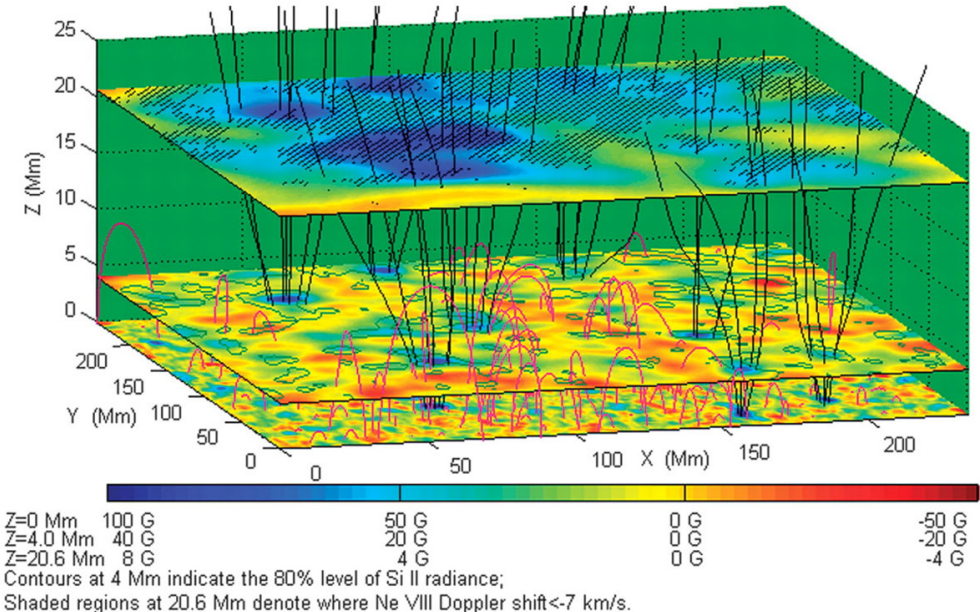

Fig. 30 The linear force-free coronal magnetic field model shows open (black) and closed (red) field lines. Because of the decreasing magnetic field strength, the inserted magnetic maps at 0, 4 and $20.6 \mathrm{Mm}$ correspond to a different color bar. At the 4 and $20.6 \mathrm{Mm}$ level the authors made a correlation analysis of the extrapolated magnetic field Bz and Doppler maps from SOHO/SUMER. The shaded area at $20.6 \mathrm{Mm}$ corresponds to a detected outflow faster than $7 \mathrm{~km} \mathrm{~s}^{-1}$. (Figure 5 of Tu et al. 2005. Reprinted with permission from AAAS)

\subsubsection{Contribution to the solar wind}

Hassler et al. (1999), using Doppler maps from SOHO/SUMER, investigated the relationship between the chromospheric magnetic network and plasma outflows. They showed that the solar wind is rooted at the boundaries of the cells of the magnetic network. Tu et al. (2005) went a step further and combined data from the same instrument with a potential coronal magnetic field model based on SOHO/MDI data. A 
correlation analysis of the modeled field structure, of observed Doppler-velocity and radiance maps revealed an acceleration of the solar wind at heights between five and $20 \mathrm{Mm}$ above the Sun's surface, and originating from coronal funnels (Fig. 30). Jin and Wang (2011), using Hinode/SOT-SP data were able to support the scenario described by $\mathrm{Tu}$ et al. (2005), namely that the solar wind streams along magnetic funnels and the magnetic reconnection of open and closed fields might provide energy for the acceleration of the particles.

\section{Conclusion and outlook}

Within this paper, we aimed to review our current understanding of the role of magnetic fields for the physics of the solar atmosphere, in particular the corona. In the past years, ground-based and space-borne instruments have delivered data of unprecedented spatial and temporal resolution. Together with the ever increasing sophistication of numerical techniques, this led to new insights into the nature and the dynamic evolution of the coronal magnetic field. A broad range of spatial scales is involved in coronal processes: from very small scales, at which magnetic reconnection may locally reconfigure the magnetic field, to very large scales, at which the long-term recycling of the global magnetic field takes place.

There are a number of techniques available for the measurement of the coronal magnetic field, but none has found widespread and regular use. Observations at radio wavelengths have provided by far the most direct measurements of coronal magnetic fields, augmented by polarization measurements in coronal lines in the infrared for a relatively small number of cases. More frequent are observations using magnetically sensitive chromospheric lines in the infrared, which can sample layers close to the coronal base. Current limitations on the spatial and temporal resolution at radio frequencies are expected to be partly lifted once FASR starts operating. More regular observations of the coronal magnetic field vector are also envisaged with the NSO/DKIST telescope. A more indirect approach is taken by coronal seismology, which permits the magnetic field strength to be derived from the analysis of oscillating loops.

Nevertheless, by far the most accurate routine measurements are of the magnetic field in the photosphere, ranging from global scales with $S D O$ to high-resolution measurements with Sunrise.

The instabilities that might trigger magnetic reconnection are still only accessible through 3D numerical MHD models. This is also because even the present imaging and spectropolarimetric instrumentation with the highest spatial resolution (e.g., Sunrise and Hi-C), might still be far from resolving the relevant spatial scales. Besides, high spatial resolution often comes with a restricted FOV. Such restrictions are less severe for the analysis of processes observed on larger active-region scales. Coronal imaging data of sufficiently high spatial resolution and temporal cadence, in the form of SDO/AIA images, have allowed detailed analysis of the coronal dynamics in recent years. The interpretation of these observations has been increasingly aided by static force-free magnetic field models which, at the same time, were validated by the match with structures seen in the same coronal images. The force-free modeling approach is well justified in the corona above an active region owing to the low plasma $\beta$ there, 
but less suitable to model the quiet Sun, where $\beta$ is not low and non-magnetic forces have to be taken into account, e.g., with magneto-static and MHD-models.

For studying the evolution of coronal magnetic fields a strong tool is the combination of flux-transport models and extrapolation techniques. Complementary stereoscopic and tomographic methods significantly improved the ability to reconstruct the $3 \mathrm{D}$ structure of coronal loops using simultaneously observed 2D images from a number of vantage points, such as SOHO/EIT, the STEREO spacecrafts, SDO/AIA, and in the future also Solar Orbiter.

A promising approach is to combine extrapolations from photospheric fields and stereoscopy within one model. Such attempts are still in their infancy though and currently non-linear force-free extrapolations of the photospheric field vector into the corona are the state-of-the-art. The maturing of such modeling tools for the interpretation of coronal dynamics has mainly become possible due to the development and operation of advanced instruments, such as SOLIS/VSM, Hinode/SOT-SP and $S D O / H M I$ that deliver vector magnetic field maps regularly for the entire solar disk and active-region scans with both high time cadence and high spatial resolution. In addition, forward MHS and MHD modeling techniques proved their strength in the ability to compute synthetic spectra characteristic of ARs which quantitatively reproduced spectra recorded with, e.g., SOHO/SUMER. Recent milestones using one or the other tools individually, or combination with each other, were discussed throughout this review. Besides having shed light on some of the hidden dynamics of the magnetic field, indirectly accessible to us only by the analysis of coronal images, the scientific outcome in recent years naturally also changed the course of the field and uncovered and/or strengthened the importance of answering still open questions.

On small granulation scales, the permanent restructuring of line-tied, braided and/or twisted active-region magnetic fields has been a strong contender for providing a contribution to the heating of the coronal plasma to the observed temperatures. Its contribution to the release of parts of the vast amount of energy stored in the coronal active-region magnetic fields, however, is still to be determined in detail. On activeregion scales, dynamic phenomena such as flares and CMEs received great attention and have revealed some of their secrets. We are increasingly able to relate observed flare-associated energetics to specific magnetic field structures, both in space and in time. The ability to predict eruptive phenomena, however, is still in its infancy. Detailed analysis of the eruption-associated magnetic field topology and its evolution is highlighting the complex interplay between photospheric driver and coronal response, underlining that the task of attempting any forecasting is a challenging one. Similarly demanding is the prediction of the long-term behavior of activity and even more so since the importance of local eruptive processes for the cyclic global restructuring remains unclear.

Finally, the findings of recent years showed us that the magnetic structure of the solar corona, even during times of low solar activity when the global magnetic field is thought to be best represented by a "simple" dipole field, is rather complex. Consequently, much work is still needed to unravel the nature of the Sun's magnetic field, on local as well as on global scales, the latter being important to understand its contribution to the solar wind and its effects on our interplanetary environment. 
Acknowledgments We thank all colleagues who have provided us with permission to reprint their figures and have helped with discussions and comments. This work was supported by the grants Deutsches Zentrum für Luft- und Raumfahrt (DLR): 50 OC 0904, Deutsche Forschungsgemeinschaft (DFG): WI 3211/21, Fonds zur Förderung der wissenschaftlichen Forschung (FWF): P25383-N27, and by the BK21 plus program through the National Research Foundation (NRF) funded by the Ministry of Education of Korea.

Open Access This article is distributed under the terms of the Creative Commons Attribution License which permits any use, distribution, and reproduction in any medium, provided the original author(s) and the source are credited.

\section{Abbreviations}

AR(s)
CH(s)
CME(s)
ECH(s)
EUV
FOV
FASR
GOES
Hi-C
Hinode
Hinode/EIS
Hinode/SOT
Hinode/SOT-SP

KPNO

LFF

LOS

$\mathrm{MC}(\mathrm{s})$

MHD

$\mathrm{M}(\mathrm{H}) \mathrm{S}$

NSO

NSO/DIKST

NLFF

$\mathrm{PCH}(\mathrm{s})$

PIL(s)

PFSS

QSL(s)

QS

$S D O$

SDO/AIA

SDO/HMI

$\mathrm{SOHO}$
Active Region(s)

Coronal Hole(s)

Coronal Mass Ejection(s)

Equatorial Coronal Hole(s)

Extreme Ultraviolet)

Field-Of-View

Frequency Agile Solar Radiotelescope (Gary and Keller 2005)

Geostationary Operational Environmental Satellite

(http://www.goes.noaa.gov/)

High-resolution Coronal Imager (Golub et al. 2006; Cirtain et al. 2013)

Solar-B (Ichimoto and Solar-B Team 2005)

Hinode EUV Imaging Spectrometer (Culhane et al. 2007)

Hinode Solar Optical Telescope (Suematsu et al. 2008)

Hinode SOT Spectro-Polarimeter (Tsuneta et al. 2008b; Lites et al. 2013)

Kitt Peak National Observatory (http://www.noao.edu/kpno/)

Linear Force-Free

Line-Of-Sight

Magnetic Cloud(s)

Magneto-Hydro-Dynamic

Magneto-(Hydro-)Static

National Solar Observatory (http://www.nso.edu/)

NSO Daniel K. Inouye Solar Telescope (http://atst.nso.edu/)

Non-linear Force-Free

Polar Coronal Hole(s)

Polarity Inversion Line(s)

Potential Field Source Surface

Quasi-Separatrix Layer(s)

Quiet Sun

Solar Dynamics Observatory (Pesnell et al. 2012)

SDO Atmospheric Imaging Assembly (Lemen et al. 2012)

SDO Helioseismic and Magnetic Imager (Schou et al. 2012)

Solar and Heliospheric Observatory (Scherrer et al. 1995) 
SOHO/EIT

SOHO/LASCO

SOHO/MDI

SOHO/SUMER

SOLIS

SOLIS/VSM

Solar Orbiter

STEREO

STEREO/SECCHI

STEREO/SECCHICOR1(2)

STEREO/SECCHIEUVI

Sunrise

SXR(s)

Sunrise/IMaX

TEL(s)

TRACE

VTT

WIND/WAVES

Yohkoh

Yohkoh/SXT
SOHO Extreme ultraviolet Imaging Telescope

(Delaboudinière et al. 1995)

SOHO Large Angle and Spectrometric Coronagraph

(Brueckner et al. 1995)

SOHO Michelson Doppler Imager (Scherrer et al. 1995)

SOHO Solar Ultraviolet Measurements of Emitted Radiation (Wilhelm et al. 1995)

Solar Optical Long-term Investigations of the Sun (Keller et al. 2003a)

SOLIS Vector SpectroMagnetograph (Keller et al. 2003b)

(Müller et al. 2013)

Solar-TErrestrial RElations Observatory (Kaiser et al. 2008)

STEREO Sun Earth Connection Coronal and Heliospheric Investigation (Howard et al. 2002)

STEREO/SECCHI inner (outer) CORonagraph (Liu et al. 2009b)

STEREO/SECCHI Extreme UltraViolet Imager (Wuelser et al. 2004)

Solar-C (Solanki et al. 2010; Barthol et al. 2011)

Soft X-ray(s)

Sunrise Imaging Magnetograph eXperiment (Martínez Pillet et al. 2011)

Trans-Equatorial Loop(s)

Transition Region and Coronal Explorer (Handy et al. 1999)

Vacuum Tower Telescope (http://www.kis.uni-freiburg.de/)

Bougeret et al. (1995)

Solar-A (Tsuneta et al. 1991)

Yohkoh Soft X-ray Telescope (Tsuneta et al. 1991)

\section{References}

Abramenko VI, Longcope DW (2005) Distribution of the magnetic flux in elements of the magnetic field in active regions. Astrophys J 619:1160-1166. doi:10.1086/426710

Abramenko VI, Carbone V, Yurchyshyn V, Goode PR, Stein RF, Lepreti F, Capparelli V, Vecchio A (2011) Turbulent diffusion in the photosphere as derived from photospheric bright point motion. Astrophys J 743:133. doi:10.1088/0004-637X/743/2/133

Aly JJ (1989) On the reconstruction of the nonlinear force-free coronal magnetic field from boundary data. Sol Phys 120:19-48

Antiochos SK, DeVore CR (1999) The role of helicity in magnetic reconnection: 3D numerical simulations. Wash DC Am Geophys Union Geophys Mon Ser 111:187

Arnaud J (1982a) Observed polarization of the Fe XIV 5303 coronal emission line. Astron Astrophys $112: 350-354$

Arnaud J (1982b) The analysis of Fe XIV 5303 coronal emission-line polarization measurements. Astron Astrophys 116:248-254

Aschwanden MJ (2005) Physics of the solar corona. An introduction with problems and solutions, 2nd edn. Praxis Publishing Ltd., Chichester

Aschwanden MJ (2011) Solar stereoscopy and tomography. Living Rev Sol Phys 8:5. doi:10.12942/ lrsp-2011-5 
Aschwanden MJ, Boerner P (2011) Solar corona loop studies with the atmospheric imaging assembly. I. Cross-sectional temperature structure. Astrophys J 732:81. doi:10.1088/0004-637X/732/2/81

Aschwanden MJ, Sun X, Liu Y (2014) The magnetic field of active region 11158 during the 2011 February 12-17 flares: differences between photospheric extrapolation and coronal forward-fitting methods. Astrophys J 785:34. doi:10.1088/0004-637X/785/1/34

Athay RG, Querfeld CW, Smartt RN, Bommier V (1983) Vector magnetic fields in prominences. III-HeI D3 Stokes profile analysis for quiescent and eruptive prominences. Sol Phys 89:3-30. doi:10.1007/ BF00211948

Aulanier G, DeLuca EE, Antiochos SK, McMullen RA, Golub L (2000) The topology and evolution of the Bastille day flare. Astrophys J 540:1126-1142. doi:10.1086/309376

Aulanier G, Pariat E, Démoulin P (2005) Current sheet formation in quasi-separatrix layers and hyperbolic flux tubes. Astron Astrophys 444:961-976. doi:10.1051/0004-6361:20053600

Bai T (2003) Periodicities in solar flare occurrence: analysis of cycles 19-23. Astrophys J 591:406-415. doi: $10.1086 / 375295$

Baker D, van Driel-Gesztelyi L, Mandrini CH, Démoulin P, Murray MJ (2009) Magnetic reconnection along quasi-separatrix layers as a driver of ubiquitous active region outflows. Astrophys J 705:926935. doi:10.1088/0004-637X/705/1/926

Bao SD, Sakurai T, Suematsu Y (2002) The sources of magnetic field twist in solar active regions. Astrophys J 573:445-453

Barthol P, Gandorfer A, Solanki SK, Schüssler M, Chares B, Curdt W, Deutsch W, Feller A, Germerott D, Grauf B, Heerlein K, Hirzberger J, Kolleck M, Meller R, Müller R, Riethmüller TL, Tomasch G, Knölker M, Lites BW, Card G, Elmore D, Fox J, Lecinski A, Nelson P, Summers R, Watt A, Bonet JA, Schmidt W, Domingo V, Álvarez-Herrero A, Sabau-Graziati L, Widani C, Haberler P, Härtel K, Kampf D, Levin T, Sanz-Andrés A, Schmidt E (2011) The sunrise mission. Sol Phys 268:1-34. doi:10. 1007/s11207-010-9662-9

Baty H, Heyvaerts J (1996) Electric current concentration and kink instability in line-tied coronal loops. Astron Astrophys 308:935-950

Baumann I, Schmitt D, Schüssler M, Solanki SK (2004) Evolution of the large-scale magnetic field on the solar surface: a parameter study. Astron Astrophys 426:1075-1091. doi:10.1051/0004-6361:20048024

Beck C, Choudhary DP, Rezaei R (2014) A three-dimensional view of the thermal structure in a superpenumbral canopy. Astrophys J 788:183. doi:10.1088/0004-637X/788/2/183

Bellan PM (1999) Magnetic helicity and relaxation: theory. In: Brown MR, Canfield RC, Pevtsov AA (eds) Helicity in space and laboratory plasmas, pp 119-128

Bellan PM (2006) Fundamentals of plasma physics. Cambridge University Press, Cambridge

Benz AO (2008) Flare observations. Living Rev Sol Phys 5(1). http://www.livingreviews.org/lrsp-2008-1

Berdyugina SV, Usoskin IG (2003) Active longitudes in sunspot activity: century scale persistence. Astron Astrophys 405:1121-1128. doi:10.1051/0004-6361:20030748

Berger MA (1984) Rigorous new limits on magnetic helicity dissipation in the solar corona. Geophys Astrophys Fluid Dyn 30:79-104

Berger TE, Title AM (1996) On the dynamics of small-scale solar magnetic elements. Astrophys J 463:365. doi:10.1086/177250

Berger MA, Ruzmaikin A (2000) Rate of helicity production by solar rotation. J Geophys Res 105:1048110490. doi:10.1029/1999JA900392

Berger MA, Asgari-Targhi M (2009) Self-organized braiding and the structure of coronal loops. Astrophys J 705:347-355. doi:10.1088/0004-637X/705/1/347

Bernasconi PN, Rust DM, Hakim D (2005) Advanced automated solar filament detection and characterization code: description, performance, and results. Sol Phys 228:97-117. doi:10.1007/ s11207-005-2766-y

Berton R, Sakurai T (1985) Stereoscopic determination of the three-dimensional geometry of coronal magnetic loops. Sol Phys 96:93-111. doi:10.1007/BF00239795

Bieber JW, Rust DM (1995) The escape of magnetic flux from the sun. Astrophys J 453:911. doi:10.1086/ 176451

Bleybel A, Amari T, van Driel-Gesztelyi L, Leka KD (2002) Global budget for an eruptive active region. I. Equilibrium reconstruction approach. Astron Astrophys 395:685-695. doi:10.1051/0004-6361: 20021332

Bogdan TJ, Low BC (1986) The three-dimensional structure of magnetostatic atmospheres. II-Modeling the large-scale corona. Astrophys J 306:271-283. doi:10.1086/164341 
Bogod VM, Stupishin AG, Yasnov LV (2012) On magnetic fields of active regions at coronal heights. Sol Phys 276:61-73. doi:10.1007/s11207-011-9850-2

Borrero JM, Ichimoto K (2011) Magnetic structure of sunspots. Living Rev Sol Phys 8(4). doi:10.12942/ lrsp-2011-4. http://www.livingreviews.org/lrsp-2011-4

Bougeret J-L, Kaiser ML, Kellogg PJ, Manning R, Goetz K, Monson SJ, Monge N, Friel L, Meetre CA, Perche C, Sitruk L, Hoang S (1995) Waves: the radio and plasma wave investigation on the wind spacecraft. Space Sci Rev 71:231-263. doi:10.1007/BF00751331

Bray RJ, Loughhead RE (1964) Sunspots. Chapman \& Hall, London

Bray RJ, Cram LE, Durrant CJ, Loughhead RE (1991) Plasma loops in the solar corona. Cambridge astrophysics series. Cambridge University Press, Cambridge

Brekke P, Kjeldseth-Moe O, Brynildsen N, Maltby P, Haugan SVH, Harrison RA, Thompson WT, Pike CD (1997) Flows and dynamics in the corona observed with the coronal diagnostic spectrometer (cds). Sol Phys 170:163-177. doi:10.1023/A:1004945702259

Briand C, Solanki SK (1995) Empirical models of solar magnetic elements: constraints imposed by $\mathrm{MgI}$ Stokes profiles. Astron Astrophys 299:596

Brosius JW, White SM (2006) Radio measurements of the height of strong coronal magnetic fields above sunspots at the solar limb. Astrophys J Lett 641:L69-L72. doi:10.1086/503774

Brosius JW, Davila JM, Thomas RJ, White SM (1997) Coronal magnetography of a solar active region using coordinated SERTS and VLA observations. Astrophys J 488:488-498

Brown DS, Nightingale RW, Alexander D, Schrijver CJ, Metcalf TR, Shine RA, Title AM, Wolfson CJ (2003) Observations of rotating sunspots from TRACE. Sol Phys 216:79-108

Brueckner GE, Bartoe J-DF (1983) Observations of high-energy jets in the corona above the quiet sun, the heating of the corona, and the acceleration of the solar wind. Astrophys J 272:329-348. doi:10.1086/ 161297

Brueckner GE, Howard RA, Koomen MJ, Korendyke CM, Michels DJ, Moses JD, Socker DG, Dere KP, Lamy PL, Llebaria A, Bout MV, Schwenn R, Simnett GM, Bedford DK, Eyles CJ (1995) The large angle spectroscopic coronagraph (LASCO). Sol Phys 162:357-402. doi:10.1007/BF00733434

Bruls JHMJ, Solanki SK (1995) Infrared lines as probes of solar magnetic features. IX. MgI $12 \mu \mathrm{m}$ diagnostics of solar plage. Astron Astrophys 293:240-251

Bruno R, Carbone V (2013) The solar wind as a turbulence laboratory. Living Rev Sol Phys 10(2). doi:10. 12942/lrsp-2013-2. http://www.livingreviews.org/lrsp-2013-2

Buente M, Solanki SK, Steiner O (1993) Centre-to-limb variation of the Stokes V asymmetry in solar magnetic flux tubes. Astron Astrophys 268:736-748

Bühler D, Lagg A, Solanki SK (2013) Quiet Sun magnetic fields observed by Hinode: support for a local dynamo. Astron Astrophys 555:A33. doi:10.1051/0004-6361/201321152

Burlaga LF (1988) Magnetic clouds and force-free fields with constant alpha. J Geophys Res 93:7217-7224. doi:10.1029/JA093iA07p07217

Burlaga L, Sittler E, Mariani F, Schwenn R (1981) Magnetic loop behind an interplanetary shock—Voyager, Helios, and IMP 8 observations. J Geophys Res 86:6673-6684. doi:10.1029/JA086iA08p06673

Caligari P, Moreno-Insertis F, Schussler M (1995) Emerging flux tubes in the solar convection zone. 1: Asymmetry, tilt, and emergence latitude. Astrophys J 441:886-902. doi:10.1086/175410

Cameron R, Vögler A, Schüssler M (2011) Decay of a simulated mixed-polarity magnetic field in the solar surface layers. Astron Astrophys 533:A86. doi:10.1051/0004-6361/201116974

Canfield RC, Reardon KP, Leka KD, Shibata K, Yokoyama T, Shimojo M (1996) H alpha surges and X-ray jets in AR 7260. Astrophys J 464:1016. doi:10.1086/177389

Canfield RC, Hudson HS, McKenzie DE (1999) Sigmoidal morphology and eruptive solar activity. Geophys Res Lett 26:627-630. doi:10.1029/1999GL900105

Cargill PJ (2009) Coronal magnetism: difficulties and prospects. Space Sci Rev 144:413-421. doi:10.1007/ s11214-008-9446-9

Carlsson M, Stein RF (1997) Formation of solar calcium H and K bright grains. Astrophys J 481:500-514

Carmichael H (1964) A process for flares. NASA Spec Publ 50:451

Carroll JA (1933) Zones of apparent inhibition of sunspots on the solar disc. Nature 131:548-549. doi:10. 1038/131548b0

Centeno R, Socas-Navarro H, Lites B, Kubo M, Frank Z, Shine R, Tarbell T, Title A, Ichimoto K, Tsuneta S, Katsukawa Y, Suematsu Y, Shimizu T, Nagata S (2007) Emergence of small-scale magnetic loops in the quiet-Sun internetwork. Astrophys J Lett 666:L137-L140. doi:10.1086/521726 
Chae J, Moon Y-J, Park Y-D (2004) Determination of magnetic helicity content of solar active regions from SOHO/MDI magnetograms. Sol Phys 223:39-55. doi:10.1007/s11207-004-0938-9

Chandra R, Schmieder B, Aulanier G, Malherbe JM (2009) Evidence of magnetic helicity in emerging flux and associated flare. Sol Phys 258:53-67. doi:10.1007/s11207-009-9392-z

Chandra R, Pariat E, Schmieder B, Mandrini CH, Uddin W (2010) How can a negative magnetic helicity active region generate a positive helicity magnetic cloud? Sol Phys 261:127-148. doi:10.1007/ s11207-009-9470-2

Charbonneau P (2010) Dynamo models of the solar cycle. Living Rev Sol Phys 7(3). doi:10.12942/ lrsp-2010-3. http://www.livingreviews.org/lrsp-2010-3

Chen PF (2011) Coronal mass ejections: models and their observational basis. Living Rev Sol Phys 8(1). http://www.livingreviews.org/lrsp-2011-1

Chen AQ, Wang JX (2012) Quantifying solar superactive regions with vector magnetic field observations. Astron Astrophys 543:A49. doi:10.1051/0004-6361/201118037

Chen J, Wang H, Zirin H, Ai G (1994) Observations of vector magnetic fields in flaring active regions. Sol Phys 154:261-273. doi:10.1007/BF00681099

Chen J, Bao S, Zhang H (2006) A statistical study of transequatorial loops. Sol Phys 235:281-294. doi:10. 1007/s11207-006-0072-y

Chen J, Lundstedt H, Zhang H (2010) Statistical study of twist values of transequatorial loops and the relationship with flares. Adv Space Res 45:537-540. doi:10.1016/j.asr.2009.10.005

Chen C, Wang Y, Shen C, Ye P, Zhang J, Wang S (2011) Statistical study of coronal mass ejection source locations: 2. Role of active regions in CME production. J Geophys Res (Space Phys) 116(A15):A12108. doi:10.1029/2011JA016844

Chesny DL, Oluseyi HM, Orange NB (2013) Non-potential fields in the quiet Sun network: extremeultraviolet and magnetic footpoint observations. Astrophys J Lett 778:L17. doi:10.1088/2041-8205/ 778/1/L17

Cheung MCM, Isobe H (2014) Flux emergence (theory). Living Rev Sol Phys 11:3. doi:10.12942/ lrsp-2014-3

Chidambara Aiyar PR (1932) Two longitudinal zones of apparent inhibition of sunspots on the solar disc. Mon Not R Astron Soc 93:150

Chitta LP, Kariyappa R, van Ballegooijen AA, DeLuca EE, Solanki SK (2014) Nonlinear force-free field modeling of the solar magnetic carpet and comparison with SDO/HMI and sunrise/IMaX observations. Astrophys J 793:112. doi:10.1088/0004-637X/793/2/112

Cho K-S, Park S-H, Marubashi K, Gopalswamy N, Akiyama S, Yashiro S, Kim R-S, Lim E-K (2013) Comparison of helicity signs in interplanetary CMEs and their solar source regions. Sol Phys 284:105127. doi:10.1007/s11207-013-0224-9

Choe GS, Cheng CZ (2000) A model of solar flares and their homologous behavior. Astrophys J 541:449467. doi:10.1086/309415

Choudhary DP, Sakurai T, Venkatakrishnan P (2001) Chromospheric magnetic field of solar active regions. Astrophys J 560:439-444. doi:10.1086/322501

Choudhary DP, Venkatakrishnan P, Gosain S (2002) On magnetic flux imbalance in solar active regions. Astrophys J 573:851-856. doi:10.1086/340754

Cirtain JW, Golub L, Lundquist L, van Ballegooijen A, Savcheva A, Shimojo M, DeLuca E, Tsuneta S, Sakao T, Reeves K, Weber M, Kano R, Narukage N, Shibasaki K (2007) Evidence for Alfvén waves in solar X-ray jets. Science 318:1580-1582. doi:10.1126/science.1147050

Cirtain JW, Golub L, Winebarger AR, de Pontieu B, Kobayashi K, Moore RL, Walsh RW, Korreck KE, Weber M, McCauley P, Title A, Kuzin S, Deforest CE (2013) Energy release in the solar corona from spatially resolved magnetic braids. Nature 493:501-503. doi:10.1038/nature11772

Close RM, Parnell CE, Longcope DW, Priest ER (2004) Recycling of the solar corona's magnetic field. Astrophys J Lett 612:L81-L84. doi:10.1086/424659

Close RM, Parnell CE, Longcope DW, Priest ER (2005) Coronal flux recycling times. Sol Phys 231:45-70. doi:10.1007/s11207-005-6878-1

Cranmer SR (2009) Coronal holes. Living Rev Sol Phys 6:3. http://www.livingreviews.org/lrsp-2009-3

Culhane JL, Harra LK, James AM, Al-Janabi K, Bradley LJ, Chaudry RA, Rees K, Tandy JA, Thomas P, Whillock MCR, Winter B, Doschek GA, Korendyke CM, Brown CM, Myers S, Mariska J, Seely J, Lang J, Kent BJ, Shaughnessy BM, Young PR, Simnett GM, Castelli CM, Mahmoud S, MapsonMenard H, Probyn BJ, Thomas RJ, Davila J, Dere K, Windt D, Shea J, Hagood R, Moye R, Hara H, 
Watanabe T, Matsuzaki K, Kosugi T, Hansteen V, Wikstol Ø (2007) The EUV imaging spectrometer for Hinode. Sol Phys 243:19-61. doi:10.1007/s01007-007-0293-1

Danilovic S, Beeck B, Pietarila A, Schüssler M, Solanki SK, Martínez Pillet V, Bonet JA, del Toro Iniesta JC, Domingo V, Barthol P, Berkefeld T, Gandorfer A, Knölker M, Schmidt W, Title AM (2010a) Transverse component of the magnetic field in the solar photosphere observed by SUNRISE. Astrophys J Lett 723:L149-L153. doi:10.1088/2041-8205/723/2/L149

Danilovic S, Schüssler M, Solanki SK (2010b) Probing quiet Sun magnetism using MURaM simulations and Hinode/SP results: support for a local dynamo. Astron Astrophys 513:A1. doi:10.1051/0004-6361/ 200913379

Dasi-Espuig M, Solanki SK, Krivova NA, Cameron R, Peñuela T (2010) Sunspot group tilt angles and the strength of the solar cycle. Astron Astrophys 518:A7. doi:10.1051/0004-6361/201014301

Davila JM (1994) Solar tomography. Astrophys J 423:871. doi:10.1086/173864

de la Cruz Rodríguez J, Socas-Navarro H (2011) Are solar chromospheric fibrils tracing the magnetic field? Astron Astrophys 527:L8. doi:10.1051/0004-6361/201016018

De Pontieu B, McIntosh SW, Carlsson M, Hansteen VH, Tarbell TD, Schrijver CJ, Title AM, Shine RA, Tsuneta S, Katsukawa Y, Ichimoto K, Suematsu Y, Shimizu T, Nagata S (2007) Chromospheric Alfvénic waves strong enough to power the solar wind. Science 318:1574-1577. doi:10.1126/science. 1151747

De Rosa ML, Schrijver CJ, Barnes G, Leka KD, Lites BW, Aschwanden MJ, Amari T, Canou A, McTiernan JM, Régnier S, Thalmann JK, Valori G, Wheatland MS, Wiegelmann T, Cheung MCM, Conlon PA, Fuhrmann M, Inhester B, Tadesse T (2009) A critical assessment of nonlinear force-free field modeling of the solar corona for active region 10953. Astrophys J 696:1780-1791. doi:10.1088/0004-637X/ $696 / 2 / 1780$

de Wijn AG, Stenflo JO, Solanki SK, Tsuneta S (2009) Small-scale solar magnetic fields. Space Sci Rev 144:275-315. doi:10.1007/s11214-008-9473-6

DeForest CE (2007) On the size of structures in the solar corona. Astrophys J 661:532-542. doi:10.1086/ 515561

DeForest CE, Hoeksema JT, Gurman JB, Thompson BJ, Plunkett SP, Howard R, Harrison RC, Hassler DM (1997) Polar plume anatomy: results of a coordinated observation. Sol Phys 175:393-410. doi:10. 1023/A:1004955223306

Del Zanna G, Bromage BJI (1999) The elephant's trunk: spectroscopic diagnostics applied to SOHO/CDS observations of the August 1996 equatorial coronal hole. J Geophys Res 104:9753-9766. doi:10.1029/ 1998JA900067

Del Zanna G, Mason HE (2003) Solar active regions: SOHO/CDS and TRACE observations of quiescent coronal loops. Astron Astrophys 406:1089-1103. doi:10.1051/0004-6361:20030791

Delaboudinière J-P, Artzner GE, Brunaud J, Gabriel AH, Hochedez JF, Millier F, Song XY, Au B, Dere KP, Howard RA, Kreplin R, Michels DJ, Moses JD, Defise JM, Jamar C, Rochus P, Chauvineau JP, Marioge JP, Catura RC, Lemen JR, Shing L, Stern RA, Gurman JB, Neupert WM, Maucherat A, Clette F, Cugnon P, van Dessel EL (1995) EIT: Extreme-ultraviolet Imaging Telescope for the SoHO mission. Sol Phys 162(1-2):291-312. doi:10.1007/BF00733432

Démoulin P (2006) Extending the concept of separatrices to QSLs for magnetic reconnection. Adv Space Res 37:1269-1282. doi:10.1016/j.asr.2005.03.085

Démoulin P (2007) Recent theoretical and observational developments in magnetic helicity studies. Adv Space Res 39:1674-1693. doi:10.1016/j.asr.2006.12.037

Démoulin P, Berger MA (2003) Magnetic energy and helicity fluxes at the photospheric level. Sol Phys 215:203-215

Démoulin P, Pariat E (2009) Modelling and observations of photospheric magnetic helicity. Adv Space Res 43:1013-1031. doi:10.1016/j.asr.2008.12.004

Démoulin P, Bagala LG, Mandrini CH, Henoux JC, Rovira MG (1997) Quasi-separatrix layers in solar flares. II. Observed magnetic configurations. Astron Astrophys 325:305-317

Démoulin P, Mandrini CH, Van Driel-Gesztelyi L, Lopez Fuentes MC, Aulanier G (2002) The magnetic helicity injected by shearing motions. Sol Phys 207:87-110

DeVore CR (2000) Magnetic helicity generation by solar differential rotation. Astrophys J 539:944-953. doi:10.1086/309274

Dowdy JF Jr, Rabin D, Moore RL (1986) On the magnetic structure of the quiet transition region. Sol Phys 105:35-45. doi:10.1007/BF00156374 
Dun J, Kurokawa H, Ishii TT, Liu Y, Zhang H (2007) Evolution of magnetic nonpotentiality in NOAA AR 10486. Astrophys J 657:577-591. doi:10.1086/510373

Durrant CJ (1988) The atmosphere of the sun. Hilger, Bristol

Durrant CJ, McCloughan J (2004) A method of evolving synoptic maps of the solar magnetic field, II. Comparison with observations of the polar fields. Sol Phys 219:55-78. doi:10.1023/B:SOLA.0000021830. 88336.86

Einaudi G, van Hoven G (1983) The stability of coronal loops-finite-length and pressure-profile limits. Sol Phys 88:163-177. doi:10.1007/BF00196185

Evershed J (1909) Radial movement in sun-spots. Mon Not R Astron Soc 69:454

Fan Y, Gibson SE (2003) The emergence of a twisted magnetic flux tube into a preexisting coronal arcade. Astrophys J Lett 589:L105-L108. doi:10.1086/375834

Feng L, Inhester B, Solanki SK, Wiegelmann T, Podlipnik B, Howard RA, Wuelser J-P (2007) First stereoscopic coronal loop reconstructions from STEREO SECCHI images. Astrophys J Lett 671:L205-L208. doi: $10.1086 / 525525$

Feng L, Inhester B, Solanki SK, Wilhelm K, Wiegelmann T, Podlipnik B, Howard RA, Plunkett SP, Wuelser JP, Gan WQ (2009) Stereoscopic polar plume reconstructions from STEREO/SECCHI images. Astrophys J 700:292-301. doi:10.1088/0004-637X/700/1/292

Feng L, Wiegelmann T, Su Y, Inhester B, Li YP, Sun XD, Gan WQ (2013) Magnetic energy partition between the coronal mass ejection and flare from AR 11283. Astrophys J 765:37. doi:10.1088/0004-637X/ $765 / 1 / 37$

Fletcher L, Dennis BR, Hudson HS, Krucker S, Phillips K, Veronig A, Battaglia M, Bone L, Caspi A, Chen Q, Gallagher P, Grigis PT, Ji H, Liu W, Milligan RO, Temmer M (2011) An observational overview of solar flares. Space Sci Rev 159:19-106. doi:10.1007/s11214-010-9701-8

Floyd O, Lamy P, Llebaria A (2014) The interaction between coronal mass ejections and streamers: a statistical view over 15 years (1996-2010). Sol Phys 289:1313-1339. doi:10.1007/s11207-013-0379-4

Forbes TG (2000) A review on the genesis of coronal mass ejections. J Geophys Res 105:23153-23166. doi:10.1029/2000JA000005

Forbes TG, Linker JA, Chen J, Cid C, Kóta J, Lee MA, Mann G, Mikić Z, Potgieter MS, Schmidt JM, Siscoe GL, Vainio R, Antiochos SK, Riley P (2006) CME theory and models. Space Sci Rev 123:251-302. doi:10.1007/s11214-006-9019-8

Foukal P (2004) Solar astrophysics, 2nd edn. Wiley, New York

Gabriel AH (1976) A magnetic model of the solar transition region. Philos Trans R Soc Lond A 281:339-352

Gabriel AH, Bely-Dubau F, Lemaire P (2003) The contribution of polar plumes to the fast solar wind. Astrophys J 589:623-634. doi:10.1086/374416

Gaizauskas V (1985) Observations of the fine structure of the chromosphere. In: Lites BW (eds) Chromospheric diagnostics and modelling, pp 25-49

Gary GA (2001) Plasma-beta above a solar active region: rethinking the paradigm. Sol Phys 203:71-86

Gary DE, Keller CU (2005) Solar and space weather radiophysics: current status and future developments. Astrophysics and space science library. Springer, Dordrecht

Gary GA, Moore RL, Hagyard MJ, Haisch BM (1987) Nonpotential features observed in the magnetic field of an active region. Astrophys J 314:782-794. doi:10.1086/165104

Georgoulis MK, Rust DM, Pevtsov AA, Bernasconi PN, Kuzanyan KM (2009) Solar magnetic helicity injected into the heliosphere: magnitude, balance, and periodicities over solar cycle 23. Astrophys $\mathrm{J}$ Lett 705:L48-L52. doi:10.1088/0004-637X/705/1/L48

Gibson SE, Fan Y, Mandrini C, Fisher G, Demoulin P (2004) Observational consequences of a magnetic flux rope emerging into the corona. Astrophys J 617:600-613. doi:10.1086/425294

Gilchrist SA, Wheatland MS, Leka KD (2012) The free energy of NOAA solar active region AR 11029. Sol Phys 276:133-160. doi:10.1007/s11207-011-9878-3

Giovanelli RG (1980) An exploratory two-dimensional study of the coarse structure of network magnetic fields. Sol Phys 68:49-69. doi:10.1007/BF00153266

Giovanelli RG, Jones HP (1982) The three-dimensional structure of atmospheric magnetic fields in two active regions. Sol Phys 79:267-278. doi:10.1007/BF00146244

Glencross WM (1974) Formation of holes in the solar corona. Nature 250:717-719. doi:10.1038/250717a0

Golub L, Krieger AS, Harvey JW, Vaiana GS (1977) Magnetic properties of X-ray bright points. Sol Phys 53:111-121. doi:10.1007/BF02260212 
Golub L, Cirtain J, DeLuca E, Nystrom G, Kankelborg C, Klumpar D, Longcope D, Martens P (2006) The Hi-C sounding rocket experiment. In: AAS/solar physics division meeting \#37. Bulletin of the American Astronomical Society, vol 38, p 226

Golub L, Krieger AS, Silk JK, Timothy AF, Vaiana GS (1974) Solar X-ray bright points. Astrophys J Lett 189:L93. doi:10.1086/181472

Gopalswamy N, Hanaoka Y, Kosugi T, Lepping RP, Steinberg JT, Plunkett S, Howard RA, Thompson BJ, Gurman J, Ho G, Nitta N, Hudson HS (1998) On the relationship between coronal mass ejections and magnetic clouds. Geophys Res Lett 25:2485-2488. doi:10.1029/98GL50757

Gosain S (2012) Evidence for collapsing fields in the corona and photosphere during the 2011 February 15 X2.2 flare: SDO/AIA and HMI observations. Astrophys J 749:85. doi:10.1088/0004-637X/749/1/85

Green LM, Démoulin P, Mandrini CH, Van Driel-Gesztelyi L (2003) How are emerging flux, flares and CMEs related to magnetic polarity imbalance in MDI data? Sol Phys 215:307-325

Grossmann-Doerth U, Pahlke K-D, Schüssler M (1987) Spurious variation of photospheric magnetic flux. Astron Astrophys 176:139-145

Grossmann-Doerth U, Schüssler M, Steiner O (1998) Convective intensification of solar surface magnetic fields: results of numerical experiments. Astron Astrophys 337:928-939

Gudiksen BV, Nordlund $\AA$ (2005) An ab initio approach to the solar coronal heating problem. Astrophys J 618:1020-1030. doi:10.1086/426063

Guenther E, Mattig W (1991) High resolution line profiles in quiet and plage regions of the sun. Astron Astrophys 243:244-250

Guglielmino SL, Martínez Pillet V, Bonet JA, del Toro Iniesta JC, Bellot Rubio LR, Solanki SK, Schmidt W, Gandorfer A, Barthol P, Knölker M (2012) The frontier between small-scale bipoles and ephemeral regions in the solar photosphere: emergence and decay of an intermediate-scale bipole observed with SUNRISE/IMaX. Astrophys J 745:160. doi:10.1088/0004-637X/745/2/160

Gyenge N, Baranyi T, Ludmány A (2014) Migration and extension of solar active longitudinal zones. Sol Phys 289:579-591. doi:10.1007/s11207-013-0424-3

Hagenaar HJ (2001) Ephemeral regions on a sequence of full-disk Michelson Doppler Imager magnetograms. Astrophys J 555:448-461. doi:10.1086/321448

Hagenaar HJ, Schrijver CJ, Title AM (2003) The properties of small magnetic regions on the solar surface and the implications for the solar dynamo(s). Astrophys J 584:1107-1119. doi:10.1086/345792

Hagyard MJ, Rabin DM (1986) Measurement and interpretation of magnetic shear in solar active regions. Adv Space Res 6:7-16. doi:10.1016/0273-1177(86)90105-5

Hagyard MJ, Moore RL, Emslie AG (1984a) The role of magnetic field shear in solar flares. Adv Space Res 4:71-80. doi:10.1016/0273-1177(84)90162-5

Hagyard MJ, Teuber D, West EA, Smith JB (1984b) A quantitative study relating observed shear in photospheric magnetic fields to repeated flaring. Sol Phys 91:115-126. doi:10.1007/BF00213618

Hale GE (1908a) On the probable existence of a magnetic field in Sun-spots. Astrophys J 28:315. doi:10. $1086 / 141602$

Hale GE (1908b) Solar vortices (Contributions from the Mt. Wilson Solar Observatory, No. 26). Astrophys J 28:100. doi:10.1086/141581

Hale GE (1925) Nature of the hydrogen vortices surrounding sunspots. Publ Astron Soc Pac 37:268-270. doi:10.1086/123497

Hale GE, Nicholson SB (1925) The law of sunspot polarity. Astrophys J 62:270-300. doi:10.1086/142933

Hale GE, Ellerman F, Nicholson SB, Joy AH (1919) The magnetic polarity of sunspots. Astrophys J 49:153178. doi:10.1086/142452

Handy BN, Acton LW, Kankelborg CC, Wolfson CJ, Akin DJ, Bruner ME, Caravalho R, Catura RC, Chevalier R, Duncan DW, Edwards CG, Feinstein CN, Freeland SL, Friedlaender FM, Hoffmann CH, Hurlburt NE, Jurcevich BK, Katz NL, Kelly GA, Lemen JR, Levay M, Lindgren RW, Mathur DP, Meyer SB, Morrison SJ, Morrison MD, Nightingale RW, Pope TP, Rehse RA, Schrijver CJ, Shine RA, Shing L, Strong KT, Tarbell TD, Title AM, Torgerson DD, Golub L, Bookbinder JA, Caldwell D, Cheimets PN, Davis WN, Deluca EE, McMullen RA, Warren HP, Amato D, Fisher R, Maldonado H, Parkinson C (1999) The transition region and coronal explorer. Sol Phys 187:229-260. doi:10.1023/ A:1005166902804

Hansteen VH, De Pontieu B, Rouppe van der Voort L, van Noort M, Carlsson M (2006) Dynamic fibrils are driven by magnetoacoustic shocks. Astrophys J Lett 647:L73-L76. doi:10.1086/507452 
Hao Q, Guo Y, Dai Y, Ding MD, Li Z, Zhang XY, Fang C (2012) Understanding the white-light flare on 2012 March 9: evidence of a two-step magnetic reconnection. Astron Astrophys 544:L17. doi:10. 1051/0004-6361/201219941

Harra LK, Matthews SA, van Driel-Gesztelyi L (2003) Evidence of flaring in a transequatorial loop on the Sun. Astrophys J Lett 598:L59-L62. doi:10.1086/380558

Harvey J, Hall D (1971) Magnetic fields measured with the $10830 \AA$ HeI line. In: Howard R (ed) Solar magnetic fields. IAU symposium, vol 43, p 279

Harvey KL, Martin SF (1973) Ephemeral active regions. Sol Phys 32:389-402. doi:10.1007/BF00154951

Harvey JW, Sheeley NR Jr (1979) Coronal holes and solar magnetic fields. Space Sci Rev 23:139-158. doi:10.1007/BF00173808

Harvey KL, Zwaan C (1993) Properties and emergence of bipolar active regions. Sol Phys 148:85-118. doi:10.1007/BF00675537

Hassler DM, Dammasch IE, Lemaire P, Brekke P, Curdt W, Mason HE, Vial J-C, Wilhelm K (1999) Solar wind outflow and the chromospheric magnetic network. Science 283:810. doi:10.1126/science.283. 5403.810

He H, Wang H, Yan Y, Chen PF, Fang C (2014) Variations of the 3-D coronal magnetic field associated with the X3.4-class solar flare event of AR 10930. J Geophys Res (Space Phys) 119:3286-3315. doi:10. 1002/2013JA019157

Heyvaerts J, Priest ER (1984) Coronal heating by reconnection in DC current systems. A theory based on Taylor's hypothesis. Astron Astrophys 137:63-78

Hirayama T (1974) Theoretical model of flares and prominences. I: Evaporating flare model. Sol Phys 34:323-338. doi:10.1007/BF00153671

Hood AW, Priest ER (1981) Critical conditions for magnetic instabilities in force-free coronal loops. Geophys Astrophys Fluid Dyn 17:297-318. doi:10.1080/03091928108243687

Hornig G, Rastätter L (1997) The role of helicity in the reconnection process. Adv Space Res 19:1789-1792. doi:10.1016/S0273-1177(97)00077-X

Howard RA, Moses JD, Socker DG, Dere KP, Cook JW, Secchi Consortium (2002) Sun earth connection coronal and heliospheric investigation (SECCHI). Adv Space Res 29:2017-2026. doi:10.1016/ S0273-1177(02)00147-3

Hu YQ, Feng XS, Wu ST, Song WB (2008) Three-dimensional MHD modeling of the global corona throughout solar cycle 23. J Geophys Res (Space Phys) 113:A03106. doi:10.1029/2007JA012750

Hudson HS (2000) Implosions in coronal transients. Astrophys J Lett 531:L75-L77. doi:10.1086/312516

Hudson HS, Bougeret J-L, Burkepile J (2006) Coronal mass ejections: overview of observations. Space Sci Rev 123:13-30. doi:10.1007/s11214-006-9009-x

Hundhausen AJ (1993) Sizes and locations of coronal mass ejections-SMM observations from 1980 and 1984-1989. J Geophys Res 98:13177. doi:10.1029/93JA00157

Ichimoto K, Solar-B Team (2005) The solar-B mission. J Korean Astron Soc 38:307-310

Innes DE, Genetelli A, Attie R, Potts HE (2009) Quiet Sun mini-coronal mass ejections activated by supergranular flows. Astron Astrophys 495:319-323. doi:10.1051/0004-6361:200811011

Innes DE, McIntosh SW, Pietarila A (2010) STEREO quadrature observations of coronal dimming at the onset of mini-CMEs. Astron Astrophys 517:L7. doi:10.1051/0004-6361/201014366

Insley JE, Moore V, Harrison RA (1995) The differential rotation of the corona as indicated by coronal holes. Sol Phys 160:1-18. doi:10.1007/BF00679089

Isobe H, Proctor MRE, Weiss NO (2008) Convection-driven emergence of small-scale magnetic fields and their role in coronal heating and solar wind acceleration. Astrophys J Lett 679:L57-L60. doi:10.1086/ 589150

Ito H, Tsuneta S, Shiota D, Tokumaru M, Fujiki K (2010) Is the polar region different from the quiet region of the Sun? Astrophys J 719:131-142. doi:10.1088/0004-637X/719/1/131

Ivanov EV (2007) Active longitudes: structure, dynamics, and rotation. Adv Space Res 40:959-969. doi:10. 1016/j.asr.2007.02.093

Jafarzadeh S, Cameron RH, Solanki SK, Pietarila A, Feller A, Lagg A, Gandorfer A (2014a) Migration of Ca II H bright points in the internetwork. Astron Astrophys 563:A101. doi:10.1051/0004-6361/ 201323011

Jafarzadeh S, Solanki SK, Lagg A, Bellot Rubio LR, van Noort M, Feller A, Danilovic S (2014b) Inclinations of small quiet-Sun magnetic features based on a new geometric approach. Astron Astrophys 569:A105. doi:10.1051/0004-6361/201423414 
Janse ÅM, Low BC (2007) Coronal hydromagnetic implosions. Astron Astrophys 472:957-965. doi:10. 1051/0004-6361:20077532

Ji H, Huang G, Wang H (2007) The relaxation of sheared magnetic fields: a contracting process. Astrophys J 660:893-900. doi:10.1086/513017

Jiang J, Choudhuri AR, Wang J (2007) A new explanation for the origin of trans-equatorial loops based on a dynamo model. Sol Phys 245:19-25. doi:10.1007/s11207-007-9033-3

Jiang J, Cameron RH, Schmitt D, Schüssler M (2011) The solar magnetic field since 1700. II. Physical reconstruction of total, polar and open flux. Astron Astrophys 528:A83. doi:10.1051/0004-6361/ 201016168

Jiang R-L, Fang C, Chen P-F (2012) Numerical simulation of solar microflares in a canopy-type magnetic configuration. Astrophys J 751:152. doi:10.1088/0004-637X/751/2/152

Jiang J, Hathaway DH, Cameron RH, Solanki SK, Gizon L, Upton L (2014) Magnetic flux transport at the solar surface. Space Sci Rev. doi:10.1007/s11214-014-0083-1

Jin C, Wang J (2011) Vector magnetic fields of a solar polar region. Astrophys J 732:4. doi:10.1088/ 0004-637X/732/1/4

Jing J, Wiegelmann T, Suematsu Y, Kubo M, Wang H (2008) Changes of magnetic structure in 3D associated with the X3.4 flare of 2006 December 13. Astrophys J Lett 676:L81-L84. doi:10.1086/587058

Jing J, Chen PF, Wiegelmann T, Xu Y, Park S-H, Wang H (2009) Temporal evolution of free magnetic energy associated with four X-class flares. Astrophys J 696:84-90. doi:10.1088/0004-637X/696/1/84

Jing J, Tan C, Yuan Y, Wang B, Wiegelmann T, Xu Y, Wang H (2010) Free magnetic energy and flare productivity of active regions. Astrophys J 713:440-449. doi:10.1088/0004-637X/713/1/440

Jing J, Yuan Y, Reardon K, Wiegelmann T, Xu Y, Wang H (2011) Nonpotentiality of chromospheric fibrils in NOAA active regions 11092 and 9661. Astrophys J 739:67. doi:10.1088/0004-637X/739/2/67

Jones HP (1985) Recent studies of magnetic canopies. Aust J Phys 38:919-928

Jones HP, Giovanelli RG (1982) Magnetograph response to canopy-type fields. Sol Phys 79:247-266. doi:10.1007/BF00146243

Joshi B, Veronig A, Cho K-S, Bong S-C, Somov BV, Moon Y-J, Lee J, Manoharan PK, Kim Y-H (2009) Magnetic reconnection during the two-phase evolution of a solar eruptive flare. Astrophys J 706:14381450. doi:10.1088/0004-637X/706/2/1438

Judge P (2006) Observations of the solar chromosphere. In: Leibacher J, Stein RF, Uitenbroek H (eds), Solar MHD theory and observations: a high spatial resolution perspective. Astronomical society of the pacific conference series, vol 354, p 259

Judge PG (2009) On magnetic field "reconstruction". Astron Astrophys 493:1121-1123. doi:10.1051/ 0004-6361:200810767

Kaiser ML, Kucera TA, Davila JM, Cyr OC, St Guhathakurta M, Christian E (2008) The STEREO mission: an introduction. Space Sci Rev 136:5-16. doi:10.1007/s11214-007-9277-0

Kaltman TI, Bogod VM, Stupishin AG, Yasnov LV (2012) The altitude structure of the coronal magnetic field of AR 10933. Astron Rep 56:790-799. doi:10.1134/S1063772912100022

Kamio S, Hara H, Watanabe T, Curdt W (2009) Distribution of jets and magnetic fields in a coronal hole. Astron Astrophys 502:345-353. doi:10.1051/0004-6361/200811125

Keller CU, Harvey JW, Giampapa MS (2003a) SOLIS: an innovative suite of synoptic instruments. In: Keil SL, Avakyan SV (eds) Innovative telescopes and instrumentation for solar astrophysics, vol 4853, pp 194-204

Keller CU, Harvey JW, The Solis Team (2003b) The SOLIS vector-spectromagnetograph. In: TrujilloBueno J, Sanchez Almeida J (eds) Solar polarization. Astronomical society of the pacific conference series, vol 307, pp 13-22

Klimchuk JA (2000) Cross-sectional properties of coronal loops. Sol Phys 193:53-75. doi:10.1023/A: 1005210127703

Klimchuk JA (2006) On solving the coronal heating problem. Sol Phys 234:41-77. doi:10.1007/ s11207-006-0055-Z

Klimchuk JA, Lemen JR, Feldman U, Tsuneta S, Uchida Y (1992) Thickness variations along coronal loops observed by the soft X-ray telescope on YOHKOH. Publ Astron Soc Jpn 44:L181-L185

Kopp RA, Pneuman GW (1976) Magnetic reconnection in the corona and the loop prominence phenomenon. Sol Phys 50:85-98. doi:10.1007/BF00206193

Krall KR, Smith JB Jr, Hagyard MJ, West EA, Cummings NP (1982) Vector magnetic field evolution, energy storage, and associated photospheric velocity shear within a flare-productive active region. Sol Phys 79:59-75. doi:10.1007/BF00146973 
Kramar M, Inhester B, Solanki SK (2006) Vector tomography for the coronal magnetic field. I. Longitudinal Zeeman effect measurements. Astron Astrophys 456:665-673. doi:10.1051/0004-6361:20064865

Kramar M, Inhester B, Lin H, Davila J (2013) Vector tomography for the coronal magnetic field. II. Hanle effect measurements. Astrophys J 775:25. doi:10.1088/0004-637X/775/1/25

Kramar M, Airapetian V, Mikić Z, Davila J (2014) 3D coronal density reconstruction and retrieving the magnetic field structure during solar minimum. Sol Phys. doi:10.1007/s11207-014-0525-7

Krucker S, Hurford GJ, Lin RP (2003) Hard X-ray source motions in the 2002 July 23 gamma-ray flare. Astrophys J Lett 595:L103-L106. doi:10.1086/378840

Kuckein C, Martínez Pillet V, Centeno R (2012) An active region filament studied simultaneously in the chromosphere and photosphere. I. Magnetic structure. Astron Astrophys 539:A131. doi:10.1051/ 0004-6361/201117675

Kumar P, Park S-H, Cho K-S, Bong S-C (2013) Multiwavelength study of a solar eruption from AR NOAA 11112 I. Flux emergence, sunspot rotation and triggering of a solar flare. Sol Phys 282:503-521. doi:10.1007/s11207-012-0174-7

Kusano K, Maeshiro T, Yokoyama T, Sakurai T (2002) Measurement of magnetic helicity injection and free energy loading into the solar corona. Astrophys J 577:501-512. doi:10.1086/342171

Kusano K, Maeshiro T, Yokoyama T, Sakurai T (2004) The trigger mechanism of solar flares in a coronal arcade with reversed magnetic shear. Astrophys J 610:537-549. doi:10.1086/421547

Labonte BJ (1979) Activity in the quiet sun. I-Observations of macrospicules in H-alpha and D3. Sol Phys 61:283-296. doi:10.1007/BF00150413

LaBonte BJ, Georgoulis MK, Rust DM (2007) Survey of magnetic helicity injection in regions producing X-class flares. Astrophys J 671:955-963. doi:10.1086/522682

Labrosse N, Heinzel P, Vial J-C, Kucera T, Parenti S, Gunár S, Schmieder B, Kilper G (2010) Physics of solar prominences: I-spectral diagnostics and non-LTE modelling. Space Sci Rev 151:243-332. doi:10.1007/s11214-010-9630-6

Lagg A, Solanki SK, Riethmüller TL, Martínez Pillet V, Schüssler M, Hirzberger J, Feller A, Borrero JM, Schmidt W, del Toro Iniesta JC, Bonet JA, Barthol P, Berkefeld T, Domingo V, Gandorfer A, Knölker M, Title AM (2010) Fully resolved quiet-Sun magnetic flux tube observed with the SUNRISE/IMAX instrument. Astrophys J Lett 723:L164-L168. doi:10.1088/2041-8205/723/2/L164

Lamb DA, Howard TA, DeForest CE, Parnell CE, Welsch BT (2013) Solar magnetic tracking. IV. The death of magnetic features. Astrophys J 774:127. doi:10.1088/0004-637X/774/2/127

Leamon RJ, Canfield RC, Jones SL, Lambkin K, Lundberg BJ, Pevtsov AA (2004) Helicity of magnetic clouds and their associated active regions. J Geophys Res (Space Phys) 109:A05106. doi:10.1029/ 2003JA010324

Lee J, McClymont AN, Mikić Z, White SM, Kundu MR (1998) Coronal currents, magnetic fields, and heating in a solar active region. Astrophys J 501:853-865. doi:10.1086/305851

Lee J, White SM, Kundu MR, Mikić Z, McClymont AN (1999) A test for coronal magnetic field extrapolations. Astrophys J 510:413-421. doi:10.1086/306556

Leenaarts J, Carlsson M, Rouppe van der Voort L (2012) The formation of the H $\alpha$ line in the solar chromosphere. Astrophys J 749:136. doi:10.1088/0004-637X/749/2/136

Leighton RB (1964) Transport of magnetic fields on the Sun. Astrophys J 140:1547. doi:10.1086/148058

Lemen JR, Title AM, Akin DJ, Boerner PF, Chou C, Drake JF, Duncan DW, Edwards CG, Friedlaender FM, Heyman GF, Hurlburt NE, Katz NL, Kushner GD, Levay M, Lindgren RW, Mathur DP, McFeaters EL, Mitchell S, Rehse RA, Schrijver CJ, Springer LA, Stern RA, Tarbell TD, Wuelser J-P, Wolfson CJ, Yanari C, Bookbinder JA, Cheimets PN, Caldwell D, Deluca EE, Gates R, Golub L, Park S, Podgorski WA, Bush RI, Scherrer PH, Gummin MA, Smith P, Auker G, Jerram P, Pool P, Soufli R, Windt DL, Beardsley S, Clapp M, Lang J, Waltham N (2012) The atmospheric imaging assembly (AIA) on the solar dynamics observatory (SDO). Sol Phys 275:17-40. doi:10.1007/s11207-011-9776-8

Leroy JL (1977) On the intensity of magnetic field in quiescent prominences. Astron Astrophys 60:79-84

Leroy JL, Bommier V, Sahal-Brechot S (1983) The magnetic field in the prominences of the polar crown. Sol Phys 83:135-142. doi:10.1007/BF00148248

Li J (2011) Active longitudes revealed by large-scale and long-lived coronal streamers. Astrophys J 735:130. doi:10.1088/0004-637X/735/2/130

Li YP, Gan WQ (2006) The oscillatory shrinkage in TRACE $195 \AA$ Aloops during a flare impulsive phase. Astrophys J Lett 644:L97-L100. doi:10.1086/505576

Li J, Ulrich RK (2012) Long-term measurements of sunspot magnetic tilt angles. Astrophys J 758:115. doi:10.1088/0004-637X/758/2/115 
Li KJ, Zhong SH, Ding YJ, Bai JM, Li QY (1995) The sunspotless flare on April 12, 1991 and the evolution of the neighboring filaments. Astron Astrophys Suppl Ser 109:347-353

Lin H, Rimmele T (1999) The granular magnetic fields of the quiet Sun. Astrophys J 514:448-455. doi:10. $1086 / 306925$

Lin H, Penn MJ, Tomczyk S (2000) A new precise measurement of the coronal magnetic field strength. Astrophys J Lett 541:L83-L86

Lin H, Kuhn JR, Coulter R (2004) Coronal magnetic field measurements. Astrophys J Lett 613:L177-L180. doi:10.1086/425217

Lin J, Li J, Ko Y-K, Raymond JC (2009) Investigation of thickness and electrical resistivity of the current sheets in solar eruptions. Astrophys J 693:1666-1677. doi:10.1088/0004-637X/693/2/1666

Lionello R, Riley P, Linker JA, Mikić Z (2005) The effects of differential rotation on the magnetic structure of the solar corona: magnetohydrodynamic simulations. Astrophys J 625:463-473. doi:10.1086/429268

Lites BW (2005) Magnetic flux ropes in the solar photosphere: the vector magnetic field under active region filaments. Astrophys J 622:1275-1291. doi:10.1086/428080

Lites BW, Elmore DF, Seagraves P, Skumanich AP (1993) Stokes profile analysis and vector magnetic fields. VI. Fine-scale structure of a sunspot. Astrophys J 418:928-942

Lites BW, Kubo M, Socas-Navarro H, Berger T, Frank Z, Shine R, Tarbell T, Title A, Ichimoto K, Katsukawa Y, Tsuneta S, Suematsu Y, Shimizu T, Nagata S (2008) The horizontal magnetic flux of the quiet-Sun internetwork as observed with the Hinode spectro-polarimeter. Astrophys J 672:1237-1253. doi:10. $1086 / 522922$

Lites BW, Akin DL, Card G, Cruz T, Duncan DW, Edwards CG, Elmore DF, Hoffmann C, Katsukawa Y, Katz N, Kubo M, Ichimoto K, Shimizu T, Shine RA, Streander KV, Suematsu A, Tarbell TD, Title AM, Tsuneta S (2013) The Hinode spectro-polarimeter. Sol Phys 283:579-599. doi:10.1007/ s11207-012-0206-3

Liu J, Zhang H (2006) The magnetic field, horizontal motion and helicity in a fast emerging flux region which eventually forms a delta spot. Sol Phys 234:21-40. doi:10.1007/s11207-006-2091-0

Liu Y, Lin H (2008) Observational test of coronal magnetic field models. I. Comparison with potential field model. Astrophys J 680:1496-1507. doi:10.1086/588645

Liu R, Wang H (2009) Coronal implosion and particle acceleration in the wake of a filament eruption. Astrophys J Lett 703:L23-L28

Liu R, Wang H (2010) Fast contraction of coronal loops at the flare peak. Astrophys J Lett 714:L41-L46. doi:10.1088/2041-8205/714/1/L41

Liu S, Su JT (2014) Multi-channel observations of plasma outflows and the associated small-scale magnetic field cancellations on the edges of an active region. Astrophys Space Sci 351:417-425. doi:10.1007/ s10509-014-1853-7

Liu C, Deng N, Liu Y, Falconer D, Goode PR, Denker C, Wang H (2005) Rapid change of $\delta$ spot structure associated with seven major flares. Astrophys J 622:722-736. doi:10.1086/427868

Liu R, Wang H, Alexander D (2009a) Implosion in a coronal eruption. Astrophys J 696:121-135

Liu Y, Luhmann JG, Lin RP, Bale SD, Vourlidas A, Petrie GJD (2009b) Coronal mass ejections and global coronal magnetic field reconfiguration. Astrophys J Lett 698:L51-L55. doi:10.1088/0004-637X/698/ $1 / \mathrm{L} 51$

Liu R, Lee J, Wang T, Stenborg G, Liu C, Wang H (2010) A reconnecting current sheet imaged in a solar flare. Astrophys J Lett 723:L28-L33. doi:10.1088/2041-8205/723/1/L28

Liu R, Wang T-J, Lee J, Stenborg G, Liu C, Park S-H, Wang H-M (2011a) Observing the reconnection region in a transequatorial loop system. Res Astron Astrophys 11:1209-1228. doi:10.1088/1674-4527/11/ $10 / 009$

Liu S, Zhang HQ, Su JT (2011b) Comparison of nonlinear force-free field and potential field in the quiet Sun. Sol Phys 270:89-107. doi:10.1007/s11207-011-9741-6

Liu R, Liu C, Török T, Wang Y, Wang H (2012) Contracting and erupting components of sigmoidal active regions. Astrophys J 757:150. doi:10.1088/0004-637X/757/2/150

Liu Y, Hoeksema JT, Bobra M, Hayashi K, Schuck PW, Sun X (2014) Magnetic helicity in emerging solar active regions. Astrophys J 785:13. doi:10.1088/0004-637X/785/1/13

Livi SHB, Wang J, Martin SF (1985) The cancellation of magnetic flux. I-On the quiet sun. Aust J Phys 38:855-873

Livingston W, Harvey JW, Malanushenko OV, Webster L (2006) Sunspots with the strongest magnetic fields. Sol Phys 239:41-68. doi:10.1007/s11207-006-0265-4 
Longcope DW (2005) Topological methods for the analysis of solar magnetic fields. Living Rev Sol Phys 2(7). http://www.livingreviews.org/lrsp-2005-7

López Fuentes MC, Demoulin P, Mandrini CH, van Driel-Gesztelyi L (2000) The counterkink rotation of a non-hale active region. Astrophys J 544:540-549. doi:10.1086/317180

López Fuentes MC, Démoulin P, Mandrini CH, Pevtsov AA, van Driel-Gesztelyi L (2003) Magnetic twist and writhe of active regions. On the origin of deformed flux tubes. Astron Astrophys 397:305-318. doi:10.1051/0004-6361:20021487

Low BC (1977a) Evolving force-free magnetic fields. I-The development of the preflare stage. Astrophys J 212:234-242. doi:10.1086/155042

Low BC (1977b) Evolving force-free magnetic fields. II-Stability of field configurations and the accompanying motion of the medium. Astrophys J 217:988-998. doi:10.1086/155645

Low BC (1991) Three-dimensional structures of magnetostatic atmospheres. III-A general formulation. Astrophys J 370:427-434. doi:10.1086/169829

Luoni ML, Mandrini CH, Cristiani GD, Démoulin P (2007) The magnetic field topology associated with two M flares. Adv Space Res 39:1382-1388. doi:10.1016/j.asr.2007.02.005

Luoni ML, Démoulin P, Mandrini CH, van Driel-Gesztelyi L (2011) Twisted flux tube emergence evidenced in longitudinal magnetograms: magnetic tongues. Sol Phys 270:45-74. doi:10.1007/ s11207-011-9731-8

Mackay DH, van Ballegooijen AA (2006) Models of the large-scale corona. I. Formation, evolution, and liftoff of magnetic flux ropes. Astrophys J 641:577-589. doi:10.1086/500425

Mackay D, Yeates A (2012) The Sun's global photospheric and coronal magnetic fields: observations and models. Living Rev Sol Phys 9:6. doi:10.12942/lrsp-2012-6

Mackay DH, Karpen JT, Ballester JL, Schmieder B, Aulanier G (2010) Physics of solar prominences: II-magnetic structure and dynamics. Space Sci Rev 151:333-399. doi:10.1007/s11214-010-9628-0

Maclean RC, Büchner J, Priest ER (2009) Relationship between the topological skeleton, current concentrations, and 3D magnetic reconnection sites in the solar atmosphere. Astron Astrophys 501:321-333. doi:10.1051/0004-6361:20078664

Maeshiro T, Kusano K, Yokoyama T, Sakurai T (2005) A statistical study of the correlation between magnetic helicity injection and soft X-ray activity in solar active regions. Astrophys J 620:1069-1084. doi:10. $1086 / 426806$

Magara T, Longcope DW (2003) Injection of magnetic energy and magnetic helicity into the solar atmosphere by an emerging magnetic flux tube. Astrophys J 586:630-649. doi:10.1086/367611

Malanushenko A, Schrijver CJ (2013) On the anisotropy in expansion of magnetic flux tubes in the solar corona. Astrophys J 775:120. doi:10.1088/0004-637X/775/2/120

Malanushenko A, Schrijver CJ, DeRosa ML, Wheatland MS (2014) Using coronal loops to reconstruct the magnetic field of an active region before and after a major flare. Astrophys J 783:102. doi:10.1088/ 0004-637X/783/2/102

Mandrini CH, Démoulin P, van Driel-Gesztelyi L, Green LM, López Fuentes MC (2004) Magnetic helicity budget of solar-active regions from the photosphere to magnetic clouds. Astrophys Space Sci 290:319344. doi:10.1023/B:ASTR.0000032533.31817.0e

Mandrini CH, Pohjolainen S, Dasso S, Green LM, Démoulin P, van Driel-Gesztelyi L, Copperwheat C, Foley C (2005) Interplanetary flux rope ejected from an X-ray bright point. The smallest magnetic cloud source-region ever observed. Astron Astrophys 434:725-740

Marsch E (2006) Kinetic physics of the solar corona and solar wind. Living Rev Sol Phys 3:1. doi:10.12942/ lrsp-2006-1

Marsch E, Wiegelmann T, Xia LD (2004) Coronal plasma flows and magnetic fields in solar active regions. Combined observations from SOHO and NSO/Kitt peak. Astron Astrophys 428:629-645. doi:10. 1051/0004-6361:20041060

Martin SF (1984) Dynamic signatures of quiet sun magnetic fields. In: Keil SL (eds) Small-scale dynamical processes in quiet stellar atmospheres, p 30

Martin SF (1988) The identification and interaction of network, intranetwork, and ephemeral-region magnetic fields. Sol Phys 117:243-259. doi:10.1007/BF00147246

Martin SF, Harvey KH (1979) Ephemeral active regions during solar minimum. Sol Phys 64:93-108. doi:10. 1007/BF00151118

Martin SF, Livi SHB, Wang J (1985) The cancellation of magnetic flux. II-In a decaying active region. Aust J Phys 38:929-959 
Martínez González MJ, Collados M, Ruiz Cobo B, Solanki SK (2007) Low-lying magnetic loops in the solar internetwork. Astron Astrophys 469:L39-L42. doi:10.1051/0004-6361:20077505

Martínez González MJ, Bellot Rubio LR (2009) Emergence of small-scale magnetic loops through the quiet solar atmosphere. Astrophys J 700:1391-1403. doi:10.1088/0004-637X/700/2/1391

Martínez González MJ, Manso Sainz R, Asensio Ramos A, Bellot Rubio LR (2010) Small magnetic loops connecting the quiet surface and the hot outer atmosphere of the Sun. Astrophys J Lett 714:94-97. doi:10.1088/2041-8205/714/1/L94

Martínez Pillet V (2013) Solar surface and atmospheric dynamics. The photosphere. Space Sci Rev 178:141162. doi:10.1007/s11214-013-9967-8

Martínez Pillet V, Lites BW, Skumanich A (1997) Active region magnetic fields. I. Plage fields. Astrophys J 474:810-842

Martínez Pillet V, Del Toro Iniesta JC, Àlvarez-Herrero A, Domingo V, Bonet JA, González Fernández L, López Jiménez A, Pastor C, Gasent Blesa JL, Mellado P, Piqueras J, Aparicio B, Balaguer M, Ballesteros E, Belenguer T, Bellot Rubio LR, Berkefeld T, Collados M, Deutsch W, Feller A, Girela F, Grauf B, Heredero RL, Herranz M, Jerónimo JM, Laguna H, Meller R, Menéndez M, Morales R, Orozco Suárez D, Ramos G, Reina M, Ramos JL, Rodríguez P, Sánchez A, Uribe-Patarroyo N, Barthol P, Gandorfer A, Knoelker M, Schmidt W, Solanki SK, Vargas Domínguez S (2011) The imaging magnetograph eXperiment (IMaX) for the sunrise Balloon-Borne solar observatory. Sol Phys 268:57-102. doi:10.1007/s11207-010-9644-y

Masson S, Pariat E, Aulanier G, Schrijver CJ (2009) The nature of flare ribbons in coronal null-point topology. Astrophys J 700:559-578. doi:10.1088/0004-637X/700/1/559

Mathew SK, Solanki SK, Lagg A, Collados M, Borrero JM, Berdyugina S (2004) Thermal-magnetic relation in a sunspot and a map of its Wilson depression. Astron Astrophys 422:693-701. doi:10.1051/ 0004-6361:20040136

McIntosh SW, de Pontieu B, Carlsson M, Hansteen V, Boerner P, Goossens M (2011) Alfvénic waves with sufficient energy to power the quiet solar corona and fast solar wind. Nature 475:477-480. doi:10. 1038 /nature 10235

Mercier C, Chambe G (2012) Morphology of the quiet Sun between 150 and $450 \mathrm{MHz}$ as observed with the Nançay radioheliograph. Astron Astrophys 540:A18. doi:10.1051/0004-6361/201118163

Merenda L, Lagg A, Solanki SK (2011) The height of chromospheric loops in an emerging flux region. Astron Astrophys 532:A63. doi:10.1051/0004-6361/201014988

Metcalf TR, Jiao L, McClymont AN, Canfield RC, Uitenbroek H (1995) Is the solar chromospheric magnetic field force-free? Astrophys J 439:474-481

Metcalf TR, Leka KD, Mickey DL (2005) Magnetic free energy in NOAA active region 10486 on 2003 October 29. Astrophys J Lett 623:L53-L56

Metcalf TR, De Rosa ML, Schrijver CJ, Barnes G, van Ballegooijen AA, Wiegelmann T, Wheatland MS, Valori G, McTtiernan JM (2008) Nonlinear force-free modeling of coronal magnetic fields. II. Modeling a filament arcade and simulated chromospheric and photospheric vector fields. Sol Phys 247:269-299. doi:10.1007/s11207-007-9110-7

Miesch MS, Dikpati M (2014) A three-dimensional Babcock-Leighton solar dynamo model. Astrophys J Lett 785:L8. doi:10.1088/2041-8205/785/1/L8

Mikić Z, Schnack DD, van Hoven G (1990) Dynamical evolution of twisted magnetic flux tubes. IEquilibrium and linear stability. Astrophys J 361:690-700. doi:10.1086/169232

Moffatt HK (1969) The degree of knottedness of tangled vortex lines. J Fluid Mech 35:117-129. doi:10. 1017/S0022112069000991

Mok Y, Mikić Z, Lionello R, Linker JA (2008) The formation of coronal loops by thermal instability in three dimensions. Astrophys J Lett 679:L161-L165. doi:10.1086/589440

Moon Y-J, Choe GS, Yun HS, Park YD, Mickey DL (2002) Force-freeness of solar magnetic fields in the photosphere. Astrophys J 568:422-431. doi:10.1086/338891

Moore RL, Larosa TN, Orwig LE (1995) The wall of reconnection-driven magnetohydrodynamic turbulence in a large solar flare. Astrophys J 438:966-985. doi:10.1086/175140

Moreno-Insertis F, Galsgaard K (2013) Plasma jets and eruptions in solar coronal holes: a three-dimensional flux emergence experiment. Astrophys J 771:20. doi:10.1088/0004-637X/771/1/20

Müller D, Marsden RG, Cyr OC, St Gilbert HR (2013) Solar orbiter. Exploring the Sun-heliosphere connection. Sol Phys 285:25-70. doi:10.1007/s11207-012-0085-7

Nagata S, Tsuneta S, Suematsu Y, Ichimoto K, Katsukawa Y, Shimizu T, Yokoyama T, Tarbell TD, Lites BW, Shine RA, Berger TE, Title AM, Bellot Rubio LR, Orozco Suárez D (2008) Formation of solar 
magnetic flux tubes with kilogauss field strength induced by convective instability. Astrophys J Lett 677:L145-L147. doi:10.1086/588026

Nakagawa Y, Raadu MA, Billings DE, McNamara D (1971) On the topology of filaments and chromospheric fibrils near sunspots. Sol Phys 19:72-85. doi:10.1007/BF00148825

Nakamizo A, Tanaka T, Kubo Y, Kamei S, Shimazu H, Shinagawa H (2009) Development of the 3-D MHD model of the solar corona-solar wind combining system. J Geophys Res (Space Phys) 114:A07109. doi:10.1029/2008JA013844

Nakariakov VM, Verwichte E (2005) Coronal waves and oscillations. Living Rev Sol Phys 2:3. doi:10. 12942/1rsp-2005-3

Neukirch T (1995) On self-consistent three-dimensional analytic solutions of the magnetohydrostatic equations. Astron Astrophys 301:628

Neupert WM (1968) Comparison of solar X-ray line emission with microwave emission during flares. Astrophys J Lett 153:L59-L64. doi:10.1086/180220

Nitta NV, De Rosa ML (2008) A comparison of solar open field regions found by type III radio bursts and the potential field source surface model. Astrophys J Lett 673:L207-L210. doi:10.1086/527548

Nordlund A, Stein RF, Asplund M (2009) Solar surface convection. Living Rev Sol Phys 6(2). doi:10. 12942/lrsp-2009-2. http://www.livingreviews.org/lrsp-2009-2

Ofman L (2010) Wave modeling of the solar wind. Living Rev Sol Phys 7(4). doi:10.12942/lrsp-2010-4. http://www.livingreviews.org/lrsp-2010-4

Ossendrijver M (2003) The solar dynamo. Astron Astrophys Rev 11:287-367. doi:10.1007/ s00159-003-0019-3

Owens MJ, Forsyth RJ (2013) The heliospheric magnetic field. Living Rev Sol Phys 10(5). doi:10.12942/ lrsp-2013-5. http://www.livingreviews.org/lrsp-2013-5

Parenti S (2014) Solar prominences: observations. Living Rev Sol Phys 11:1. doi:10.12942/1rsp-2014-1

Pariat E, Masson S, Aulanier G (2009) Current buildup in emerging serpentine flux tubes. Astrophys J 701:1911-1921. doi:10.1088/0004-637X/701/2/1911

Park S-H, Lee J, Choe GS, Chae J, Jeong H, Yang G, Jing J, Wang H (2008) The variation of relative magnetic helicity around major flares. Astrophys J 686:1397-1403. doi:10.1086/591117

Park S-H, Chae J, Wang H (2010) Productivity of solar flares and magnetic helicity injection in active regions. Astrophys J 718:43-51. doi:10.1088/0004-637X/718/1/43

Parker EN (1955) Hydromagnetic dynamo models. Astrophys J 122:293-314. doi:10.1086/146087

Parker EN (1978a) Hydraulic concentration of magnetic fields in the solar photosphere. VI-Adiabatic cooling and concentration in downdrafts. Astrophys J 221:368-377. doi:10.1086/156035

Parker EN (1978b) The mutual attraction of magnetic knots. Astrophys J 222:357-364. doi:10.1086/156148

Parker EN (1979) Cosmical magnetic fields: their origin and their activity. Clarendon Press, Oxford

Parker EN (1983) Magnetic neutral sheets in evolving fields-part two-formation of the solar corona. Astrophys J 264:642. doi:10.1086/160637

Parker EN (1987) Small-scale energy storage and release as the cause of the stellar X-ray corona. In: Athay G, Spicer DS (eds) NASA conference publication. NASA Conference Publication, vol 2483, pp 89-99

Parker EN (1988) Nanoflares and the solar X-ray corona. Astrophys J 330:474-479. doi:10.1086/166485

Parnell CE (2001) A model of the solar magnetic carpet. Sol Phys 200:23-45. doi:10.1023/A: 1010312014999

Parnell CE, De Moortel I (2012) A contemporary view of coronal heating. Philos Trans R Soc Lond A 370:3217-3240. doi:10.1098/rsta.2012.0113

Parnell CE, Haynes AL, Galsgaard K (2008) Recursive reconnection and magnetic skeletons. Astrophys J 675:1656-1665. doi:10.1086/527532

Parnell CE, DeForest CE, Hagenaar HJ, Johnston BA, Lamb DA, Welsch BT (2009) A power-law distribution of solar magnetic fields over more than five decades in flux. Astrophys J 698:75-82. doi:10. 1088/0004-637X/698/1/75

Parnell CE, Maclean RC, Haynes AL (2010) The detection of numerous magnetic separators in a threedimensional magnetohydrodynamic model of solar emerging flux. Astrophys J Lett 725:L214-L218. doi:10.1088/2041-8205/725/2/L214

Pelt J, Brooke JM, Korpi MJ, Tuominen I (2006) Kinematic frames and "active longitudes": does the Sun have a face? Astron Astrophys 460:875-885. doi:10.1051/0004-6361:20065399

Penn MJ (2014) Infrared solar physics. Living Rev Sol Phys 11:2. doi:10.12942/1rsp-2014-2

Penn MJ, Kuhn JR (1995) Imaging spectropolarimetry of the He I 1083 nanometer line in a flaring solar active region. Astrophys J Lett 441:L51-L54. doi:10.1086/187787 
Pesnell WD, Thompson BJ, Chamberlin PC (2012) The solar dynamics observatory (SDO). Sol Phys 275:3-15. doi:10.1007/s11207-011-9841-3

Peter H (1996) Superpenumbral vortex structures. Astrophys Lett Commun 34:77

Peter H (2002) Hierarchy of chromospheric structures and their relation to the magnetic field. In: SawayaLacoste H (ed) SOLMAG 2002. Proceedings of the magnetic coupling of the solar atmosphere Euroconference. ESA special publication, vol 505, pp 155-162

Peter H, Bingert S (2012) Constant cross section of loops in the solar corona. Astron Astrophys 548:A1. doi:10.1051/0004-6361/201219473

Peter H, Gudiksen BV, Nordlund $\AA$ (2006) Forward modeling of the corona of the Sun and solar-like stars: from a three-dimensional magnetohydrodynamic model to synthetic extreme-ultraviolet spectra. Astrophys J 638:1086-1100. doi:10.1086/499117

Peter H, Bingert S, Klimchuk JA, de Forest C, Cirtain JW, Golub L, Winebarger AR, Kobayashi K, Korreck $\mathrm{KE}$ (2013) Structure of solar coronal loops: from miniature to large-scale. Astron Astrophys 556:A104. doi:10.1051/0004-6361/201321826

Petrie GJD (2012) A spatio-temporal description of the abrupt changes in the photospheric magnetic and lorentz-force vectors during the 15 February 2011 X2.2 flare. Sol Phys, p 183. doi:10.1007/ s11207-012-0071-0

Petrie GJD, Patrikeeva I (2009) A comparative study of magnetic fields in the solar photosphere and chromosphere at equatorial and polar latitudes. Astrophys J 699:871-884. doi:10.1088/0004-637X/ $699 / 1 / 871$

Petrie GJD, Haislmaier KJ (2013) Low-latitude coronal holes, decaying active regions, and global coronal magnetic structure. Astrophys J 775:100. doi:10.1088/0004-637X/775/2/100

Petrovay K, van Driel-Gesztelyi L (1997) Making sense of sunspot decay. I. Parabolic decay law and Gnevyshev-Waldmeier relation. Sol Phys 176:249-266

Pevtsov AA (2000) Transequatorial loops in the solar corona. Astrophys J 531:553-560. doi:10.1086/ 308467

Pevtsov AA, Balasubramaniam KS (2003) Helicity patterns on the sun. Adv Space Res 32:1867-1874. doi:10.1016/S0273-1177(03)90620-X

Pevtsov AA, Canfield RC, Metcalf TR (1995) Latitudinal variation of helicity of photospheric magnetic fields. Astrophys J Lett 440:L109-L112. doi:10.1086/187773

Pevtsov AA, Canfield RC, McClymont AN (1997) On the subphotospheric origin of coronal electric currents. Astrophys J 481:973-977

Pietarila A, Hirzberger J, Zakharov V, Solanki SK (2009) Bright fibrils in Ca II K. Astron Astrophys 502:647-660. doi:10.1051/0004-6361/200811155

Pietarila A, Cameron RH, Danilovic S, Solanki SK (2011) Transport of magnetic flux from the canopy to the internetwork. Astrophys J 729:136. doi:10.1088/0004-637X/729/2/136

Platten SJ, Parnell CE, Haynes AL, Priest ER, Mackay DH (2014) The solar cycle variation of topological structures in the global solar corona. Astron Astrophys 565:A44. doi:10.1051/0004-6361/201323048

Ploner SRO, Schüssler M, Solanki SK, Gadun AS (2001) An example of reconnection and magnetic flux recycling near the solar surface. In: Sigwarth M (ed) Advanced solar polarimetry-theory, observation, and instrumentation, vol 236. Astronomical society of the pacific conference series, p 363

Pneuman GW, Kopp RA (1971) Gas-magnetic field interactions in the solar corona. Sol Phys 18:258-270. doi:10.1007/BF00145940

Priest ER (1978) The structure of coronal loops. Sol Phys 58:57-87. doi:10.1007/BF00152555

Priest ER (1982) Solar magneto-hydrodynamics. Holland, Dordrecht

Priest ER (1994) Heating of X-ray bright points and other coronal structures. Wash DC Am Geophys Union Geophys Mon Ser 84:1-13

Priest ER (2007) Coronal heating. In: Birn J, Priest ER (eds) Reconnection of magnetic fields. Cambridge University Press, Cambridge, pp 229-236

Priest E (2014) Magnetohydrodynamics of the Sun. Cambridge University Press, Cambridge

Priest ER, Démoulin P (1995) Three-dimensional magnetic reconnection without null points. 1. Basic theory of magnetic flipping. J Geophys Res 100:23443-23464. doi:10.1029/95JA02740

Priest ER, Forbes TG (2002) The magnetic nature of solar flares. Astron Astrophys Rev 10:313-377. doi:10. $1007 / \mathrm{s} 001590100013$

Priest E, Forbes T (2007) Magnetic reconnection. Cambridge University Press, Cambridge, UK

Rabin D (1992) Spatially extended measurements of magnetic field strength in solar plages. Astrophys J 391:832-844. doi:10.1086/171392 
Raouafi N-E (2005) Measurement methods for chromospheric and coronal magnetic fields. In: Innes DE, Solanki SA (eds) Chromospheric and coronal magnetic fields. ESA special publication, vol 596

Raouafi N-E, Petrie GJD, Norton AA, Henney CJ, Solanki SK (2008) Evidence for polar jets as precursors of polar plume formation. Astrophys J Lett 682:L137-L140. doi:10.1086/591125

Rappazzo AF, Velli M, Einaudi G, Dahlburg RB (2008) Nonlinear dynamics of the parker scenario for coronal heating. Astrophys J 677:1348-1366. doi:10.1086/528786

Reale F (2010) Coronal loops: observations and modeling of confined plasma. Living Rev Sol Phys 7(5). doi:10.12942/lrsp-2010-5. http://www.livingreviews.org/lrsp-2010-5

Reardon KP, Wang Y-M, Muglach K, Warren HP (2011) Evidence for two separate but interlaced components of the chromospheric magnetic field. Astrophys J 742:119. doi:10.1088/0004-637X/742/2/ 119

Régnier S, Priest ER (2007) Free magnetic energy in solar active regions above the minimum-energy relaxed state. Astrophys J Lett 669:L53-L56. doi:10.1086/523269

Régnier S, Amari T, Kersalé E (2002) 3D Coronal magnetic field from vector magnetograms: non-constantalpha force-free configuration of the active region NOAA 8151. Astron Astrophys 392:1119-1127. doi:10.1051/0004-6361:20020993

Régnier S, Fleck B, Abramenko V, Zhang H-Q (2005) Evolution of the magnetic energy budget in AR 10486 from potential and nonlinear force-free models. In: Innes DE, Lagg A, Solanki SA (eds) Chromospheric and coronal magnetic fields. ESA special publication, vol 596

Régnier S, Canfield RC (2006) Evolution of magnetic fields and energetics of flares in active region 8210. Astron Astrophys 451:319-330. doi:10.1051/0004-6361:20054171

Régnier S, Parnell CE, Haynes AL (2008) A new view of quiet-Sun topology from Hinode/SOT. Astron Astrophys 484:L47-L50. doi:10.1051/0004-6361:200809826

Requerey IS, Del Toro Iniesta JC, Bellot Rubio LR, Bonet JA, Martínez Pillet V, Solanki SK, Schmidt W (2014) The history of a quiet-Sun magnetic element revealed by IMaX/SUNRISE. Astrophys J 789:6. doi:10.1088/0004-637X/789/1/6

Richardson RS (1941) The nature of solar hydrogen vortices. Astrophys J 93:24-28. doi:10.1086/144238

Rieutord M, Rincon F (2010) The sun's supergranulation. Living Rev Sol Phys 7(2). doi:10.12942/ lrsp-2010-2. http://www.livingreviews.org/lrsp-2010-2

Riley P, Linker JA, Mikić Z, Lionello R, Ledvina SA, Luhmann JG (2006) A comparison between global solar magnetohydrodynamic and potential field source surface model results. Astrophys J 653:15101516. doi: $10.1086 / 508565$

Riley P, Lionello R, Linker JA, Mikić Z, Luhmann J, Wijaya J (2011) Global MHD modeling of the solar corona and inner heliosphere for the whole heliosphere interval. Sol Phys 274:361-377. doi:10.1007/ s1 1207-010-9698-x

Roberts WO (1945) A preliminary report on chromospheric spicules of extremely short lifetime. Astrophys J 101:136. doi:10.1086/144699

Roberts B (1990) Properties and models of photospheric flux tubes. Wash DC Am Geophys Union Geophys Mon Ser 58:113-132

Roberts B, Ulmschneider P (1997) Dynamics of flux tubes in the solar atmosphere: theory. In: Simnett GM, Alissandrakis CE, Vlahos L (eds) European meeting on solar physics. Lecture notes in physics, vol 489. Springer, Berlin, p 75. doi:10.1007/BFb0105671

Roberts B, Edwin PM, Benz AO (1984) On coronal oscillations. Astrophys J 279:857-865. doi:10.1086/ 161956

Romano P, Zuccarello F (2007) Photospheric magnetic evolution of super active regions. Astron Astrophys 474:633-637. doi:10.1051/0004-6361:20078110

Rosenthal CS, Bogdan TJ, Carlsson M, Dorch SBF, Hansteen V, McIntosh SW, McMurry A, Nordlund $\AA$, Stein RF (2002) Waves in the magnetized solar atmosphere. I. Basic processes and internetwork oscillations. Astrophys J 564:508-524. doi:10.1086/324214

Rudenko GV, Myshyakov II (2011) Gauge-invariant helicity for force-free magnetic fields in a rectangular box. Sol Phys 270:165-173. doi:10.1007/s11207-011-9743-4

Rüedi I, Solanki SK, Livingston W, Stenflo JO (1992) Infrared lines as probes of solar magnetic features. III-Strong and weak magnetic fields in plages. Astron Astrophys 263:323-338

Rüedi I, Solanki SK, Livingston WC (1995) Infrared lines as probes of solar magnetic features. X. HeI 10830A as a diagnostic of chromospheric magnetic fields. Astron Astrophys 293:252-262

Rüedi I, Keller CU, Solanki SK (1996) Measurement of the full Stokes vector of He I $10830 \AA$ A. Sol Phys 164:265-275. doi:10.1007/BF00146639 
Rust DM (1967) Magnetic fields in quiescent solar prominences. I. Observations. Astrophys J 150:313. doi: $10.1086 / 149333$

Rust DM (1994) Spawning and shedding helical magnetic fields in the solar atmosphere. Geophys Res Lett 21:241-244. doi:10.1029/94GL00003

Rust DM (1997) Magnetic helicity, MHD kink instabilities and reconnection in the corona. In Bentley RD, Mariska JT (eds) Magnetic reconnection in the solar atmosphere. Astronomical society of the pacific conference series, vol 111, p 353

Rust DM, Kumar A (1996) Evidence for helically kinked magnetic flux ropes in solar eruptions. Astrophys J Lett 464:L199-L202. doi:10.1086/310118

Rust DM, Haggerty DK, Georgoulis MK, Sheeley NR, Wang Y-M, De Rosa ML, Schrijver CJ (2008) On the solar origins of open magnetic fields in the heliosphere. Astrophys J 687:635-645. doi:10.1086/ 592017

Rutten RJ (2012) The quiet-Sun photosphere and chromosphere. Philos Trans R Soc Lond A 370:3129_ 3150. doi:10.1098/rsta.2011.0537

Rutten RJ, Uitenbroek H (1991) CA II H(2v) and K(2v) cell grains. Sol Phys 134:15-71. doi:10.1007/ BF00148739

Ruzdjak V, Vrsnak B, Brajsa R, Schroll A (1989) A comparison of H-alpha and soft X-ray characteristics of spotless and SPOT group flares. Sol Phys 123:309-316. doi:10.1007/BF00149108

Sakao T, Kano R, Narukage N, Kotoku J, Bando T, DeLuca EE, Lundquist LL, Tsuneta S, Harra LK, Katsukawa Y, Kubo M, Hara H, Matsuzaki K, Shimojo M, Bookbinder JA, Golub L, Korreck KE, Su Y, Shibasaki K, Shimizu T, Nakatani I (2007) Continuous plasma outflows from the edge of a solar active region as a possible source of solar wind. Science 318:1585-1588. doi:10.1126/science. 1147292

Sánchez Almeida J (2003) Inter-network magnetic fields observed during the minimum of the solar cycle. Astron Astrophys 411:615-621. doi:10.1051/0004-6361:20031560

Schad TA (2013) On the collective magnetic field strength and vector structure of dark umbral cores measured by the Hinode spectropolarimeter. Sol Phys. doi:10.1007/s11207-013-0412-7

Schad TA, Penn MJ, Lin H (2013) He I vector Magnetometry of field-aligned superpenumbral fibrils. Astrophys J 768:111. doi:10.1088/0004-637X/768/2/111

Schadee A, de Jager C, Svestka Z (1983) Enhanced X-ray emission above $3.5 \mathrm{keV}$ in active regions in the absence of flares. Sol Phys 89:287-305. doi:10.1007/BF00217252

Schatten KH, Wilcox JM, Ness NF (1969) A model of interplanetary and coronal magnetic fields. Sol Phys 6:442-455. doi:10.1007/BF00146478

Scherrer PH, Bogart RS, Bush RI, Hoeksema JT, Kosovichev AG, Schou J, Rosenberg W, Springer L, Tarbell TD, Title A, Wolfson CJ, Zayer I (1995) The solar oscillations investigation-Michelson Doppler Imager. Sol Phys 162:129-188. doi:10.1007/BF00733429

Schmelz JT, Holman GD, Brosius JW, Willson RF (1994) Coronal magnetic structures observing campaign. 3: Coronal plasma and magnetic field diagnostics derived from multiwaveband active region observations. Astrophys J 434:786-794. doi:10.1086/174781

Schmieder B, Guo Y, Moreno-Insertis F, Aulanier G, Yelles Chaouche L, Nishizuka N, Harra LK, Thalmann JK, Vargas Dominguez S, Liu Y (2013) Twisting solar coronal jet launched at the boundary of an active region. Astron Astrophys 559:A1. doi:10.1051/0004-6361/201322181

Schou J, Scherrer PH, Bush RI, Wachter R, Couvidat S, Rabello-Soares MC, Bogart RS, Hoeksema JT, Liu Y, Duvall TL, Akin DJ, Allard BA, Miles JW, Rairden R, Shine RA, Tarbell TD, Title AM, Wolfson CJ, Elmore DF, Norton AA, Tomczyk S (2012) Design and ground calibration of the helioseismic and magnetic imager (HMI) instrument on the solar dynamics observatory (SDO). Sol Phys 275:229-259. doi:10.1007/s11207-011-9842-2

Schrijver CJ (2009) Driving major solar flares and eruptions: a review. Adv Space Res 43:739-755. doi:10. 1016/j.asr.2008.11.004

Schrijver CJ, Zwaan C (2000) Solar and stellar magnetic activity. In: Cambridge astrophysics series, 34. Cambridge University Press, New York

Schrijver CJ, Title AM (2002) The topology of a mixed-polarity potential field, and inferences for the heating of the quiet solar corona. Sol Phys 207:223-240

Schrijver CJ, De Rosa ML (2003) Photospheric and heliospheric magnetic fields. Sol Phys 212:165-200. doi:10.1023/A:1022908504100

Schrijver CJ, Title AM (2003) The magnetic connection between the solar photosphere and the corona. Astrophys J Lett 597:L165-L168. doi:10.1086/379870 
Schrijver CJ, van Ballegooijen AA (2005) Is the quiet-Sun corona a quasi-steady, force-free environment? Astrophys J 630:552-560. doi:10.1086/431754

Schrijver CJ, Title AM, van Ballegooijen AA, Hagenaar HJ, Shine RA (1997) Sustaining the quiet photospheric network: the balance of flux emergence, fragmentation, merging, and cancellation. Astrophys J 487:424. doi:10.1086/304581

Schrijver CJ, Title AM, Berger TE, Fletcher L, Hurlburt NE, Nightingale RW, Shine RA, Tarbell TD, Wolfson J, Golub L, Bookbinder JA, Deluca EE, McMullen RA, Warren HP, Kankelborg CC, Handy $\mathrm{BN}$, de Pontieu B (1999) A new view of the solar outer atmosphere by the transition region and coronal explorer. Sol Phys 187:261-302. doi:10.1023/A:1005194519642

Schrijver CJ, De Rosa ML, Title AM (2002) What is missing from our understanding of long-term solar and heliospheric activity? Astrophys J 577:1006-1012. doi:10.1086/342247

Schrijver CJ, De Rosa ML, Metcalf TR, Liu Y, McTiernan J, Régnier S, Valori G, Wheatland MS, Wiegelmann T (2006) Nonlinear force-free modeling of coronal magnetic fields part I: a quantitative comparison of methods. Sol Phys 235:161-190. doi:10.1007/s11207-006-0068-7

Schrijver CJ, De Rosa ML, Metcalf T, Barnes G, Lites B, Tarbell T, McTiernan J, Valori G, Wiegelmann T, Wheatland MS, Amari T, Aulanier G, Démoulin P, Fuhrmann M, Kusano K, Régnier S, Thalmann JK (2008) Nonlinear force-free field modeling of a solar active region around the time of a major flare and coronal mass ejection. Astrophys J 675:1637-1644. doi:10.1086/527413

Schrijver CJ, DeRosa ML, Title AM (2010) Magnetic field topology and the thermal structure of the corona over solar active regions. Astrophys J 719:1083-1096. doi:10.1088/0004-637X/719/2/1083

Schüssler M (1984) The interchange instability of small flux tubes. Astron Astrophys 140:453-458

Schüssler M, Vögler A (2008) Strong horizontal photospheric magnetic field in a surface dynamo simulation. Astron Astrophys 481:L5-L8. doi:10.1051/0004-6361:20078998

Schwenn R (2006) Space weather: the solar perspective. Living Rev Sol Phys 3(2). doi:10.12942/ lrsp-2006-2. http://www.livingreviews.org/lrsp-2006-2

Seehafer N (1990) Electric current helicity in the solar atmosphere. Sol Phys 125:219-232. doi:10.1007/ BF00158402

Sersen M, Valnicek B (1993) Spotless flares and type II radio bursts. Sol Phys 145:339-345. doi:10.1007/ BF00690660

Sheeley NR Jr, Nash AG, Wang Y-M (1987) The origin of rigidly rotating magnetic field patterns on the sun. Astrophys J 319:481-502. doi:10.1086/165472

Sheeley NR, Wang Y-M, Harvey JW (1989) The effect of newly erupting flux on the polar coronal holes. Sol Phys 119:323-340. doi:10.1007/BF00146182

Shibata K, Magara T (2011) Solar flares: magnetohydrodynamic processes. Living Rev Sol Phys 8(6). http://www.livingreviews.org/lrsp-2011-6

Shibata K, Ishido Y, Acton LW, Strong KT, Hirayama T, Uchida Y, McAllister AH, Matsumoto R, Tsuneta S, Shimizu T, Hara H, Sakurai T, Ichimoto K, Nishino Y, Ogawara Y (1992) Observations of X-ray jets with the YOHKOH soft X-ray telescope. Publ Astron Soc Jpn 44:L173-L179

Shibata K, Nitta N, Strong KT, Matsumoto R, Yokoyama T, Hirayama T, Hudson H, Ogawara Y (1994) A gigantic coronal jet ejected from a compact active region in a coronal hole. Astrophys J Lett 431:L51L53. doi:10.1086/187470

Shibata K, Nakamura T, Matsumoto T, Otsuji K, Okamoto TJ, Nishizuka N, Kawate T, Watanabe H, Nagata S, UeNo S, Kitai R, Nozawa S, Tsuneta S, Suematsu Y, Ichimoto K, Shimizu T, Katsukawa Y, Tarbell TD, Berger TE, Lites BW, Shine RA, Title AM (2007) Chromospheric anemone jets as evidence of ubiquitous reconnection. Science 318:1591-1594. doi:10.1126/science.1146708

Shimizu T, Tsuneta S, Acton LW, Lemen JR, Ogawara Y, Uchida Y (1994) Morphology of active region transient brightenings with the YOHKOH soft X-ray telescope. Astrophys J 422:906-911. doi:10. $1086 / 173782$

Simões PJA, Fletcher L, Hudson HS, Russell AJB (2013) Implosion of coronal loops during the impulsive phase of a solar flare. Astrophys J 777:152. doi:10.1088/0004-637X/777/2/152

Sime DG (1989) Coronal mass ejection rate and the evolution of the large-scale k-coronal density distribution. J Geophys Res 94:151-158. doi:10.1029/JA094iA01p00151

Sivaraman KR, Rausaria RR, Aleem SM (1992) Magnetic shear in flaring regions. I-Quantitative evaluation of the change in shear. Sol Phys 138:353-360. doi:10.1007/BF00151919

Sobotka M, Del Moro D, Jurčák J, Berrilli F (2012) Magnetic and velocity fields of a solar pore. Astron Astrophys 537:A85. doi:10.1051/0004-6361/201117851 
Solanki SK (1993) Small-scale solar magnetic fields: an overview. Space Sci Rev 63:1-188. doi:10.1007/ BF00749277

Solanki SK (2003) Sunspots: an overview. Astron Astrophys Rev 11:153-286. doi:10.1007/ s00159-003-0018-4

Solanki SK, Steiner O (1990) How magnetic is the solar chromosphere? Astron Astrophys 234:519-529

Solanki SK, Steiner O, Uitenbroeck H (1991) Two-dimensional models of the solar chromosphere. I-The CA II K line as a diagnostic: 1.5-D radiative transfer. Astron Astrophys 250:220-234

Solanki SK, Walther U, Livingston W (1993) Infrared lines as probes of solar magnetic features. VI. The thermal-magnetic relation and Wilson depression of a simple sunspot. Astron Astrophys 277:639

Solanki SK, Montavon CAP, Livingston W (1994) Infrared lines as probes of solar magnetic features. 7: On the nature of the Evershed effect in sunspots. Astron Astrophys 283:221-231

Solanki SK, Zufferey D, Lin H, Rüedi I, Kuhn JR (1996) Infrared lines as probes of solar magnetic features. XII. Magnetic flux tubes: evidence of convective collapse? Astron Astrophys 310:L33-L36

Solanki SK, Schüssler M, Fligge M (2000) Evolution of the Sun's large-scale magnetic field since the Maunder minimum. Nature 408:445-447. doi:10.1038/408445a

Solanki SK, Schüssler M, Fligge M (2002) Secular variation of the Sun's magnetic flux. Astron Astrophys 383:706-712. doi:10.1051/0004-6361:20011790

Solanki SK, Inhester B, Schüssler M (2006) The solar magnetic field. Rep Prog Phys 69:563-668. doi:10. 1088/0034-4885/69/3/R02

Solanki SK, Lagg A, Woch J, Krupp N, Collados M (2003) Three-dimensional magnetic field topology in a region of solar coronal heating. Nature 425:692-695. doi:10.1038/nature02035

Solanki SK, Barthol P, Danilovic S, Feller A, Gandorfer A, Hirzberger J, Riethmüller TL, Schüssler M, Bonet JA, Martínez Pillet V, del Toro Iniesta JC, Domingo V, Palacios J, Knölker M, Bello González N, Berkefeld T, Franz M, Schmidt W, Title AM (2010) SUNRISE: instrument, mission, data, and first results. Astrophys J Lett 723:L127-L133. doi:10.1088/2041-8205/723/2/L127

Spirock TJ, Yurchyshyn VB, Wang H (2002) Rapid changes in the longitudinal magnetic field related to the 2001 April 2 X20 flare. Astrophys J 572:1072-1076. doi:10.1086/340431

Spruit HC (1976) Pressure equilibrium and energy balance of small photospheric fluxtubes. Sol Phys 50:269-295. doi:10.1007/BF00155292

Spruit HC (1979) Convective collapse of flux tubes. Sol Phys 61:363-378. doi:10.1007/BF00150420

Spruit HC (1981) Magnetic flux tubes. In: Jordan SD (ed) The Sun as a star. NASA SP 50. NASA Information Branch, Washington, pp 385-412

St. John CE (1913) Radial motion in Sun-spots. Astrophys J 37:322. doi:10.1086/142002

Stein RF (2012) Solar surface magneto-convection. Living Rev Sol Phys 9:4. doi:10.12942/1rsp-2012-4

Steiner O (2007) Photospheric processes and magnetic flux tubes. In: Hasan SS, Banerjee D (eds) Kodai school on solar physics. American Institute of Physics conference series, vol 919, pp 74-121. doi:10. $1063 / 1.2756784$

Steiner O, Pizzo VJ (1989) A parametric survey of model solar fluxtubes. Astron Astrophys 211:447-462

Steiner O, Grossmann-Doerth U, Schüssler M, Knölker M (1996) Polarized radiation diagnostics of magnetohydrodynamic models of the solar atmosphere. Sol Phys 164:223-242. doi:10.1007/BF00146636

Stenflo JO (1973) Magnetic-field structure of the photospheric network. Sol Phys 32:41-63. doi:10.1007/ BF00152728

Stenflo JO (1994) Solar magnetic flux at small scales (invited review). In: Schüssler M, Schmidt W (eds) Solar magnetic fields, p 301

Stenflo JO (2012) Basal magnetic flux and the local solar dynamo. Astron Astrophys 547:A93. doi:10.1051/ 0004-6361/201219833

Stenflo JO (2013) Solar magnetic fields as revealed by Stokes polarimetry. Astron Astrophys Rev 21:66. doi:10.1007/s00159-013-0066-3

Stix M (2002) The Sun: an introduction. Springer, Berlin

Strong KT et al (1994) Coronal observations from the soft X-ray telescope on YOHKOH. Adv Space Res 14:3-11. doi:10.1016/0273-1177(94)90153-8

Sturrock PA (1996) Perspectives on solar flares. In: Balasubramaniam KS, Keil SL, Smartt RN (eds) Solar drivers of the interplanetary and terrestrial disturbances. Astronomical society of the pacific conference series, vol. 95, p 42

Sturrock PA, Weber M, Wheatland MS, Wolfson R (2001) Metastable magnetic configurations and their significance for solar eruptive events. Astrophys J 548:492-496. doi:10.1086/318671 
Su Y, van Ballegooijen A, Schmieder B, Berlicki A, Guo Y, Golub L, Huang G (2009) Flare energy build-up in a decaying active region near a coronal hole. Astrophys J 704:341-353. doi:10.1088/0004-637X/ $704 / 1 / 341$

Subramanian P, Dere KP, Rich NB, Howard RA (1999) The relationship of coronal mass ejections to streamers. J Geophys Res 104:22321-22330. doi:10.1029/1999JA900252

Suematsu Y, Tsuneta S, Ichimoto K, Shimizu T, Otsubo M, Katsukawa Y, Nakagiri M, Noguchi M, Tamura T, Kato Y, Hara H, Kubo M, Mikami I, Saito H, Matsushita T, Kawaguchi N, Nakaoji T, Nagae K, Shimada S, Takeyama N, Yamamuro T (2008) The solar optical telescope of solar-B (Hinode): the optical telescope assembly. Sol Phys 249:197-220. doi:10.1007/s11207-008-9129-4

Suess ST (1982) Polar coronal plumes. Sol Phys 75:145-159. doi:10.1007/BF00153468

Sun X, Hoeksema JT, Liu Y, Chen Q, Hayashi K (2012a) A non-radial eruption in a quadrupolar magnetic configuration with a coronal null. Astrophys J 757:149. doi:10.1088/0004-637X/757/2/149

Sun X, Hoeksema JT, Liu Y, Wiegelmann T, Hayashi K, Chen Q, Thalmann J (2012b) Evolution of magnetic field and energy in a major eruptive active region based on SDO/HMI observation. Astrophys J 748:77. doi:10.1088/0004-637X/748/2/77

Svestka Z, Krieger AS, Chase RC, Howard R (1977) Transequatorial loops interconnecting McMath regions 12472 and 12474. Sol Phys 52:69-90. doi:10.1007/BF00935791

Tadesse T, Wiegelmann T, Inhester B, MacNeice P, Pevtsov A, Sun X (2013) Full-disk nonlinear force-free field extrapolation of SDO/HMI and SOLIS/VSM magnetograms. Astron Astrophys 550:A14. doi:10. 1051/0004-6361/201220044

Tadesse T, Wiegelmann T, MacNeice PJ, Inhester B, Olson K, Pevtsov A (2014) A comparison between nonlinear force-free field and potential field models using full-disk SDO/HMI magnetogram. Sol Phys 289:831-845. doi:10.1007/s11207-013-0364-y

Taylor JB (1974) Relaxation of toroidal plasma and generation of reverse magnetic fields. Phys Rev Lett 33(19):1139-1141. doi:10.1103/PhysRevLett.33.1139

Taylor JB (1986) Relaxation and magnetic reconnection in plasmas. Rev Mod Phys 58:741-763. doi:10. 1103/RevModPhys.58.741

Thalmann JK (2010) Evolution of coronal magnetic fields. PhD thesis, Technical University Braunschweig

Thalmann JK, Wiegelmann T (2008) Evolution of the flaring active region NOAA 10540 as a sequence of nonlinear force-free field extrapolations. Astron Astrophys 484:495-502. doi:10.1051/0004-6361: 200809508

Thalmann JK, Wiegelmann T, Raouafi N-E (2008) First nonlinear force-free field extrapolations of SOLIS/VSM data. Astron Astrophys 488:L71-L74. doi:10.1051/0004-6361:200810235

Thalmann JK, Inhester B, Wiegelmann T (2011) Estimating the relative helicity of coronal magnetic fields. Sol Phys 272:243-255. doi:10.1007/s11207-011-9826-2

Thalmann JK, Tiwari SK, Wiegelmann T (2014) Force-free field modeling of twist and braiding-induced magnetic energy in an active-region corona. Astrophys J 780:102. doi:10.1088/0004-637X/780/1/102

Thomas JH, Weiss NO (2004) Fine structure in sunspots. Ann Rev Astron Astrophys 42:517-548. doi:10. 1146/annurev.astro.42.010803.115226

Tian L, Alexander D (2006) Role of sunspot and sunspot-group rotation in driving sigmoidal active region eruptions. Sol Phys 233:29-43. doi:10.1007/s11207-006-2505-z

Tian L, Alexander D (2008) On the origin of magnetic helicity in the solar corona. Astrophys J 673:532-543. doi:10.1086/524129

Tian L, Liu Y, Wang J (2002) The most violent super-active regions in the 22nd and 23rd cycles. Sol Phys 209:361-374

Tian L, Alexander D, Nightingale R (2008) Origins of coronal energy and helicity in NOAA 10030. Astrophys J 684:747-756. doi:10.1086/589492

Tian H, McIntosh SW, Habbal SR, He J (2011) Observation of high-speed outflow on plume-like structures of the quiet Sun and coronal holes with solar dynamics observatory/atmospheric imaging assembly. Astrophys J 736:130. doi:10.1088/0004-637X/736/2/130

Timothy AF, Krieger AS, Vaiana GS (1975) The structure and evolution of coronal holes. Sol Phys 42:135156. doi:10.1007/BF00153291

Title AM, Schrijver CJ (1998) The Sun's magnetic carpet. In: Donahue RA, Bookbinder JA (eds) Cool stars, stellar systems, and the Sun. Astronomical society of the pacific conference series, vol 154, p 345

Title AM, Topka KP, Tarbell TD, Schmidt W, Balke C, Scharmer G (1992) On the differences between plage and quiet sun in the solar photosphere. Astrophys J 393:782-794. doi:10.1086/171545 
Title AM, Frank ZA, Shine RA, Tarbell TD, Topka KP, Scharmer G, Schmidt W (1993) On the magnetic and velocity field geometry of simple sunspots. Astrophys J 403:780-796. doi:10.1086/172249

Titov VS (2007) Pinching of coronal fields. In: Birn J, Priest ER (eds) Reconnection of magnetic fields. Cambridge University Press, Cambridge, pp 250-258

Titov VS, Démoulin P (1999) Basic topology of twisted magnetic configurations in solar flares. Astron Astrophys 351:707-720

Titov VS, Hornig G, Démoulin P (2002) Theory of magnetic connectivity in the solar corona. J Geophys Res (Space Phys) 107:1164. doi:10.1029/2001JA000278

Tiwari SK (2012) On the force-free nature of photospheric sunspot magnetic fields as observed from Hinode (SOT/SP). Astrophys J 744:65. doi:10.1088/0004-637X/744/1/65

Tiwari SK, van Noort M, Lagg A, Solanki SK (2013) Structure of sunspot penumbral filaments: a remarkable uniformity of properties. Astron Astrophys 557:A25. doi:10.1051/0004-6361/201321391

Török T, Kliem B (2003) The evolution of twisting coronal magnetic flux tubes. Astron Astrophys 406:10431059. doi:10.1051/0004-6361:20030692

Török T, Kliem B (2005) Confined and ejective eruptions of kink-unstable flux ropes. Astrophys J Lett 630:L97-L100. doi:10.1086/462412

Török T, Kliem B, Titov VS (2004) Ideal kink instability of a magnetic loop equilibrium. Astron Astrophys 413:L27-L30. doi:10.1051/0004-6361:20031691

Tsiropoula G, Tziotziou K, Kontogiannis I, Madjarska MS, Doyle JG, Suematsu Y (2012) Solar fine-scale structures. I. Spicules and other small-scale, jet-like events at the chromospheric level: observations and physical parameters. Space Sci Rev 169:181-244. doi:10.1007/s11214-012-9920-2

Tsuneta S (1996) Interacting active regions in the solar corona. Astrophys J Lett 456:L63. doi:10.1086/ 309857

Tsuneta S, Acton L, Bruner M, Lemen J, Brown W, Caravalho R, Catura R, Freeland S, Jurcevich B, Owens J (1991) The soft X-ray telescope for the SOLAR-A mission. Sol Phys 136:37-67

Tsuneta S, Ichimoto K, Katsukawa Y, Lites BW, Matsuzaki K, Nagata S, Orozco Suárez D, Shimizu T, Shimojo M, Shine RA, Suematsu Y, Suzuki TK, Tarbell TD, Title AM (2008a) The magnetic landscape of the Sun's polar region. Astrophys J 688:1374-1381. doi:10.1086/592226

Tsuneta S, Ichimoto K, Katsukawa Y, Nagata S, Otsubo M, Shimizu T, Suematsu Y, Nakagiri M, Noguchi M, Tarbell T, Title A, Shine R, Rosenberg W, Hoffmann C, Jurcevich B, Kushner G, Levay M, Lites B, Elmore D, Matsushita T, Kawaguchi N, Saito H, Mikami I, Hill LD, Owens JK (2008b) The solar optical telescope for the Hinode mission: an overview. Sol Phys 249:167-196. doi:10.1007/ s11207-008-9174-Z

Tu C-Y, Zhou C, Marsch E, Xia L-D, Zhao L, Wang J-X, Wilhelm K (2005) Solar wind origin in coronal funnels. Science 308:519-523. doi:10.1126/science.1109447

Tziotziou K, Georgoulis MK, Raouafi N-E (2012) The magnetic energy-helicity diagram of solar active regions. Astrophys J Lett 759:L4. doi:10.1088/2041-8205/759/1/L4

Tziotziou K, Georgoulis MK, Liu Y (2013) Interpreting eruptive behavior in NOAA AR 11158 via the region's magnetic energy and relative-helicity budgets. Astrophys J 772:115. doi:10.1088/0004-637X/ 772/2/115

Tziotziou K, Tsiropoula G, Georgoulis MK, Kontogiannis I (2014) Energy and helicity budgets of solar quiet regions. Astron Astrophys 564:A86. doi:10.1051/0004-6361/201323143

Uchida Y (1970) Diagnosis of Coronal Magnetic Structure by flare-associated hydromagnetic disturbances. Publ Astron Soc Jpn 22:341

Usoskin IG, Berdyugina SV, Poutanen J (2005) Preferred sunspot longitudes: non-axisymmetry and differential rotation. Astron Astrophys 441:347-352. doi:10.1051/0004-6361:20053201

Usoskin IG, Berdyugina SV, Moss D, Sokoloff DD (2007) Long-term persistence of solar active longitudes and its implications for the solar dynamo theory. Adv Space Res 40:951-958. doi:10.1016/j.asr.2006. 12.050

Valori G, Démoulin P, Pariat E (2012a) Comparing values of the relative magnetic helicity in finite volumes. Sol Phys 278:347-366. doi:10.1007/s11207-012-9951-6

Valori G, Green LM, Démoulin P, Vargas Domínguez S, van Driel-Gesztelyi L, Wallace A, Baker D, Fuhrmann M (2012b) Nonlinear force-free extrapolation of emerging flux with a global twist and serpentine fine structures. Sol Phys 278:73-97. doi:10.1007/s11207-011-9865-8

van Ballegooijen AA, Priest ER, Mackay DH (2000) Mean field model for the formation of filament channels on the Sun. Astrophys J 539:983-994. doi:10.1086/309265 
van Ballegooijen AA, Asgari-Targhi M, Cranmer SR, DeLuca EE (2011) Heating of the solar chromosphere and corona by Alfvén wave turbulence. Astrophys J 736:3. doi:10.1088/0004-637X/736/1/3

van Ballegooijen AA, Asgari-Targhi M, Berger MA (2014) On the relationship between photospheric footpoint motions and coronal heating in solar active regions. Astrophys J 787:87. doi:10.1088/0004-637X/ $787 / 1 / 87$

Van Doorsselaere T, Nakariakov VM, Young PR, Verwichte E (2008) Coronal magnetic field measurement using loop oscillations observed by Hinode/EIS. Astron Astrophys 487:L17-L20. doi:10.1051/ 0004-6361:200810186

van Driel-Gesztelyi L, Culhane JL (2009) Magnetic flux emergence, activity, eruptions and magnetic clouds: following magnetic field from the Sun to the heliosphere. Space Sci Rev 144:351-381. doi:10.1007/ s11214-008-9461-x

van Driel-Gesztelyi L, Démoulin P, Mandrini CH (2003) Observations of magnetic helicity. Adv Space Res 32:1855-1866. doi:10.1016/S0273-1177(03)90619-3

van Driel-Gesztelyi L, Culhane JL, Baker D, Démoulin P, Mandrini CH, DeRosa ML, Rouillard AP, Opitz A, Stenborg G, Vourlidas A, Brooks DH (2012) Magnetic topology of active regions and coronal holes: implications for coronal outflows and the solar wind. Sol Phys 281:237-262. doi:10.1007/ s11207-012-0076-8

van Noort M, Lagg A, Tiwari SK, Solanki SK (2013) Peripheral downflows in sunspot penumbrae. Astron Astrophys 557:A24. doi:10.1051/0004-6361/201321073

Vecchio A, Cauzzi G, Reardon KP, Janssen K, Rimmele T (2007) Solar atmospheric oscillations and the chromospheric magnetic topology. Astron Astrophys 461:L1-L4. doi:10.1051/0004-6361:20066415

Vemareddy P, Ambastha A, Maurya RA (2012a) On the role of rotating sunspots in the activity of solar active region NOAA 11158. Astrophys J 761:60. doi:10.1088/0004-637X/761/1/60

Vemareddy P, Ambastha A, Maurya RA, Chae J (2012b) On the injection of helicity by the shearing motion of fluxes in relation to flares and coronal mass ejections. Astrophys J 761:86. doi:10.1088/0004-637X/ $761 / 2 / 86$

Venkatakrishnan P (1986) Inhibition of convective collapse of solar magnetic flux tubes by radiative diffusion. Nature 322:156. doi:10.1038/322156a0

Venkatakrishnan P, Hagyard MJ, Hathaway DH (1989) Evaluation of magnetic shear in off-disk center active regions. Sol Phys 122:215-226. doi:10.1007/BF00912993

Vögler A, Schüssler M (2007) A solar surface dynamo. Astron Astrophys 465:L43-L46. doi:10.1051/ 0004-6361:20077253

Vourlidas A, Howard RA, Esfandiari E, Patsourakos S, Yashiro S, Michalek G (2010) Comprehensive analysis of coronal mass ejection mass and energy properties over a full solar cycle. Astrophys $\mathrm{J}$ 722:1522. doi:10.1088/0004-637X/722/2/1522

Vourlidas A, Howard RA, Esfandiari E, Patsourakos S, Yashiro S, Michalek G (2011) Erratum: "Comprehensive analysis of coronal mass ejection mass and energy properties over a full solar cycle". Astrophys J 730:59. doi:10.1088/0004-637X/730/1/59

Waldmeier M (1955) Ergebnisse und Probleme der Sonnenforschung (2. erweiterte Auflage). Akademischer Verlag, Geest \& Portig, Leipzig

Walsh RW, Ireland J (2003) The heating of the solar corona. Astron Astrophys Rev 12:1-41. doi:10.1007/ s00159-003-0021-9

Wang H (1992) Evolution of vector magnetic fields and the August 271990 X-3 flare. Sol Phys 140:85-98. doi:10.1007/BF00148431

Wang J (1994) Development of magnetic shear. Sol Phys 155:285-300. doi:10.1007/BF00680597

Wang J (1995) On the transport of magnetic energy and complexity to the solar corona. Acta Astrophysica Sinica 15:261-266

Wang Y-M (1998) Network activity and the evaporative formation of polar plumes. Astrophys J Lett 501:L145-L150. doi:10.1086/311445

Wang H (2006) Rapid changes of photospheric magnetic fields around flaring magnetic neutral lines. Astrophys J 649:490-497. doi:10.1086/506320

Wang Y-M (2009) Coronal holes and open magnetic flux. Space Sci Rev 144:383-399. doi:10.1007/ s11214-008-9434-0

Wang Y-M, Sheeley NR Jr (1995) Coronal plumes and their relationship to network activity. Astrophys J 452:457. doi:10.1086/176317

Wang H, Ewell MW Jr, Zirin H, Ai G (1994) Vector magnetic field changes associated with X-class flares. Astrophys J 424:436-443. doi:10.1086/173901 
Wang Y-M, Hawley SH, Sheeley NR Jr (1996) The magnetic nature of coronal holes. Science 271:464-469. doi:10.1126/science.271.5248.464

Wang J, Shi Z, Martin SF, Livi SHB (1988) The cancellation of magnetic flux on the quiet sun. Vistas Astron 31:79-83. doi:10.1016/0083-6656(88)90179-1

Wang Y-M, Lean J, Sheeley NR Jr (2000a) The long-term variation of the Sun's open magnetic flux. Geophys Res Lett 27:505-508. doi:10.1029/1999GL010744

Wang Y-M, Sheeley NR Jr, Lean J (2000b) Understanding the evolution of the Sun's open magnetic flux. Geophys Res Lett 27:621-624. doi:10.1029/1999GL010759

Wang H, Qiu J, Jing J, Spirock TJ, Yurchyshyn V, Abramenko V, Ji H, Goode PR (2004a) Evidence of rapid flux emergence associated with the M8.7 flare on, 2002 July 26. Astrophys J 605:931-937. doi:10. $1086 / 382527$

Wang J, Zhou G, Zhang J (2004b) Helicity patterns of coronal mass ejection-associated active regions. Astrophys J 615:1021-1028. doi:10.1086/424584

Wang Y-M, Sheeley NR Jr, Rich NB (2007) Coronal pseudostreamers. Astrophys J 658:1340-1348. doi:10. 1086/511416

Wang S, Liu C, Liu R, Deng N, Liu Y, Wang H (2012a) Response of the photospheric magnetic field to the X2.2 flare on 2011 February 15. Astrophys J Lett 745:L17. doi:10.1088/2041-8205/745/2/L17

Wang S, Liu C, Wang H (2012b) The relationship between the sudden change of the lorentz force and the magnitude of associated flares. Astrophys J Lett 757:L5. doi:10.1088/2041-8205/757/1/L5

Webb DF, Howard TA (2012) Coronal mass ejections: observations. Living Rev Sol Phys 9(3). http://www. livingreviews.org/lrsp-2012-3

Weber MA, Fan Y, Miesch MS (2013) A theory on the convective origins of active longitudes on solar-like stars. Astrophys J 770:149. doi:10.1088/0004-637X/770/2/149

Wedemeyer S, Freytag B, Steffen M, Ludwig H-G, Holweger H (2004) Numerical simulation of the three-dimensional structure and dynamics of the non-magnetic solar chromosphere. Astron Astrophys 414:1121-1137. doi:10.1051/0004-6361:20031682

Wedemeyer-Böhm S, Lagg A, Nordlund Å (2009) Coupling from the photosphere to the chromosphere and the corona. Space Sci Rev 144:317-350. doi:10.1007/s11214-008-9447-8

Wedemeyer-Böhm S, Scullion E, Steiner O, Rouppe van der Voort L, de La Cruz Rodriguez J, Fedun V, Erdélyi R (2012) Magnetic tornadoes as energy channels into the solar corona. Nature 486:505-508. doi:10.1038/nature 11202

White SM (2005) Radio measurements of coronal magnetic fields. In: Innes DE, Lagg A, Solanki SA (eds) Chromospheric and coronal magnetic fields. ESA special publication, vol 596

White SM, Kundu MR (1997) Radio observations of gyroresonance emission from coronal magnetic fields. Sol Phys 174:31-52

White SM, Kundu MR, Gopalswamy N (1991) Strong magnetic fields and inhomogeneity in the solar corona. Astrophys J Lett 366:L43-L46. doi:10.1086/185905

White SM, Benz AO, Christe S, Fárník F, Kundu MR, Mann G, Ning Z, Raulin J-P, Silva-Válio AVR, Saint-Hilaire P, Vilmer N, Warmuth A (2011) The relationship between solar radio and hard X-ray emission. Space Sci Rev 159:225-261. doi:10.1007/s11214-010-9708-1

Wiegelmann T (2008) Nonlinear force-free modeling of the solar coronal magnetic field. J Geophys Res (Space Phys) 113:A03SO2. doi:10.1029/2007JA012432

Wiegelmann T, Solanki SK (2004) Similarities and differences between coronal holes and the quiet sun: are loop statistics the key? Sol Phys 225:227-247. doi:10.1007/s11207-004-3747-2

Wiegelmann T, Inhester B (2006) Magnetic stereoscopy. Sol Phys 236:25-40. doi:10.1007/ s11207-006-0153-y

Wiegelmann T, Neukirch T (2006) An optimization principle for the computation of MHD equilibria in the solar corona. Astron Astrophys 457:1053-1058. doi:10.1051/0004-6361:20065281

Wiegelmann T, Sakurai T (2012) Solar force-free magnetic fields. Living Rev Sol Phys 9:5. doi:10.12942/ lrsp-2012-5

Wiegelmann T, Inhester B, Lagg A, Solanki SK (2005a) How to use magnetic field information for coronal loop identification. Sol Phys 228:67-78. doi:10.1007/s11207-005-2511-6

Wiegelmann T, Lagg A, Solanki SK, Inhester B, Woch J (2005b) Comparing magnetic field extrapolations with measurements of magnetic loops. Astron Astrophys 433:701-705. doi:10.1051/0004-6361: 20042421

Wiegelmann T, Inhester B, Sakurai T (2006) Preprocessing of vector magnetograph data for a nonlinear force-free magnetic field reconstruction. Sol Phys 233:215-232. doi:10.1007/s11207-006-2092-z 
Wiegelmann T, Neukirch T, Ruan P, Inhester B (2007) Optimization approach for the computation of magnetohydrostatic coronal equilibria in spherical geometry. Astron Astrophys 475:701-706. doi:10. 1051/0004-6361:20078244

Wiegelmann T, Thalmann JK, Schrijver CJ, Derosa ML, Metcalf TR (2008) Can we improve the preprocessing of photospheric vector magnetograms by the inclusion of chromospheric observations? Sol Phys 247:249-267. doi:10.1007/s11207-008-9130-y

Wiegelmann T, Inhester B, Feng L (2009) Solar stereoscopy—where are we and what developments do we require to progress? Annales Geophysicae 27:2925-2936. doi:10.5194/angeo-27-2925-2009

Wiegelmann T, Solanki SK, Borrero JM, Martínez Pillet V, del Toro Iniesta JC, Domingo V, Bonet JA, Barthol P, Gandorfer A, Knölker M, Schmidt W, Title AM (2010a) Magnetic loops in the quiet Sun. Astrophys J Lett 723:185-189. doi:10.1088/2041-8205/723/2/L185

Wiegelmann T, Yelles Chaouche L, Solanki SK, Lagg A (2010b) Nonlinear force-free modelling: influence of inaccuracies in the measured magnetic vector. Astron Astrophys 511:A4. doi:10.1051/0004-6361/ 200912812

Wiegelmann T, Thalmann JK, Inhester B, Tadesse T, Sun X, Hoeksema JT (2012) How should one optimize nonlinear force-free coronal magnetic field extrapolations from SDO/HMI vector magnetograms? Sol Phys 281:37-51. doi:10.1007/s11207-012-9966-z

Wiegelmann T, Solanki SK, Borrero JM, Peter H, Barthol P, Gandorfer A, Martínez Pillet V, Schmidt W, Knölker M (2013) Evolution of the fine structure of magnetic fields in the quiet Sun observations from sunrise/IMaX and extrapolations. Sol Phys 283:253-272. doi:10.1007/s11207-013-0249-0

Wilhelm K, Curdt W, Marsch E, Schühle U, Lemaire P, Gabriel A, Vial J-C, Grewing M, Huber MCE, Jordan SD, Poland AI, Thomas RJ, Kühne M, Timothy JG, Hassler DM, Siegmund OHW (1995) SUMER-Solar ultraviolet measurements of emitted radiation. Sol Phys 162:189-231. doi:10.1007/ BF00733430

Wilhelm K, Abbo L, Auchère F, Barbey N, Feng L, Gabriel AH, Giordano S, Imada S, Llebaria A, Matthaeus WH, Poletto G, Raouafi N-E, Suess ST, Teriaca L, Wang Y-M (2011) Morphology, dynamics and plasma parameters of plumes and inter-plume regions in solar coronal holes. Astron Astrophys Rev 19:35. doi:10.1007/s00159-011-0035-7

Wilkinson LK, Emslie GA, Gary GA (1989) The effects of viewing angle on the inference of magnetic shear in preflare active regions. Sol Phys 119:77-86. doi:10.1007/BF00146214

Winebarger AR, DeLuca EE, Golub L (2001) Apparent flows above an active region observed with the transition region and coronal explorer. Astrophys J Lett 553:L81-L84. doi:10.1086/320496

Winebarger AR, Walsh RW, Moore R, De Pontieu B, Hansteen V, Cirtain J, Golub L, Kobayashi K, Korreck K, DeForest C, Weber M, Title A, Kuzin S (2013) Detecting nanoflare heating events in subarcsecond inter-moss loops using Hi-C. Astrophys J 771:21. doi:10.1088/0004-637X/771/1/21

Withbroe GL, Noyes RW (1977) Mass and energy flow in the solar chromosphere and corona. Ann Rev Astron Astrophys 15:363-387. doi:10.1146/annurev.aa.15.090177.002051

Woltjer L (1958) A theorem on force-free magnetic fields. Proc Natl Acad Sci 44:489-491

Wood P, Neukirch T (2005) Electron acceleration in reconnecting current sheets. Sol Phys 226:73-95. doi:10.1007/s11207-005-5686-y

Woodard MF, Chae J (1999) Evidence for non-potential magnetic fields in the quiet Sun. Sol Phys 184:239_ 247

Wu ST, Krall KR, Hu YQ, Hagyard MJ, Smith JB Jr (1984) Modeling of energy buildup for a flare-productive region. Sol Phys 90:117-131. doi:10.1007/BF00153789

Wuelser J-P, Lemen JR, Tarbell TD, Wolfson CJ, Cannon JC, Carpenter BA, Duncan DW, Gradwohl GS, Meyer SB, Moore AS, Navarro RL, Pearson JD, Rossi GR, Springer LA, Howard RA, Moses JD, Newmark JS, Delaboudiniere J-P, Artzner GE, Auchere F, Bougnet M, Bouyries P, Bridou F, Clotaire J-Y, Colas G, Delmotte F, Jerome A, Lamare M, Mercier R, Mullot M, Ravet M-F, Song X, Bothmer V, Deutsch W (2004) EUVI: the STEREO-SECCHI extreme ultraviolet imager. In: Fineschi S, Gummin MA (eds) Telescopes and instrumentation for solar astrophysics. Society of photo-optical instrumentation engineers (SPIE) conference series, vol 5171, pp 111-122. doi:10.1117/12.506877

Xu Z, Lagg A, Solanki S, Liu Y (2012) Magnetic fields of an active region filament from full stokes analysis of Si I $1082.7 \mathrm{~nm}$ and He I $1083.0 \mathrm{~nm}$. Astrophys J 749:138. doi:10.1088/0004-637X/749/2/138

Yamamoto TT, Kusano K (2012) Preprocessing magnetic fields with chromospheric longitudinal fields. Astrophys J 752:126. doi:10.1088/0004-637X/752/2/126

Yang S, Zhang J (2014) Properties of solar ephemeral regions at the emergence stage. Astrophys J 781:7. doi:10.1088/0004-637X/781/1/7 
Yang LP, Feng XS, Xiang CQ, Liu Y, Zhao X, Wu ST (2012) Time-dependent MHD modeling of the global solar corona for year 2007: driven by daily-updated magnetic field synoptic data. J Geophys Res (Space Phys) 117:A08110. doi:10.1029/2011JA017494

Yang S, Zhang J, Li T, Ding M (2011a) Vector magnetic fields and current helicities in coronal holes and quiet regions. Astrophys J 726:49. doi:10.1088/0004-637X/726/1/49

Yang S, Zhang J, Li T, Liu Y (2011b) SDO observations of magnetic reconnection at coronal hole boundaries. Astrophys J Lett 732:L7. doi:10.1088/2041-8205/732/1/L7

Yang S, Büchner J, Santos JC, Zhang H (2013) Evolution of relative magnetic helicity: method of computation and its application to a simulated solar corona above an active region. Sol Phys. doi:10.1007/ s11207-013-0236-5

Yeates AR, Mackay DH (2012) Chirality of high-latitude filaments over solar cycle 23. Astrophys J Lett 753:L34. doi:10.1088/2041-8205/753/2/L34

Yeates AR, Muñoz-Jaramillo A (2013) Kinematic active region formation in a three-dimensional solar dynamo model. Mon Not R Astron Soc 436:3366-3379. doi:10.1093/mnras/stt1818

Yeates AR, Mackay DH, van Ballegooijen AA (2008) Modelling the global solar corona II: coronal evolution and filament chirality comparison. Sol Phys 247:103-121. doi:10.1007/s11207-007-9097-0

Yelles Chaouche L, Kuckein C, Martínez Pillet V, Moreno-Insertis F (2012) The three-dimensional structure of an active region filament as extrapolated from photospheric and chromospheric observations. Astrophys J 748:23. doi:10.1088/0004-637X/748/1/23

Yokoyama T, Shibata K (1995) Magnetic reconnection as the origin of X-ray jets and H $\alpha$ surges on the Sun. Nature 375:42-44. doi:10.1038/375042a0

Yokoyama M, Masuda S (2010) Trans-equatorial loop system arising from coronal hole boundaries through interactions between active regions and coronal holes. Sol Phys 263:135-152. doi:10.1007/ s11207-010-9525-4

Yurchyshyn VB, Wang H, Goode PR, Deng Y (2001) Orientation of the magnetic fields in interplanetary flux ropes and solar filaments. Astrophys J 563:381-388. doi:10.1086/323778

Zayer I, Stenflo JO, Keller CU, Solanki SK (1990) Dependence of the properties of solar magnetic flux tubes on filling factor. II-Results of an inversion approach. Astron Astrophys 239:356-366

Zhang M (2005) Magnetic network and canopy. In: Innes DE, Lagg A, Solanki SA (eds) Chromospheric and coronal magnetic fields. ESA special publication, vol. 596.

Zhang H, Zhang M (2000a) Similarities between chromospheric and photospheric quiet-Sun magnetograms. Sol Phys 196:269-277. doi:10.1023/A:1005255110220

Zhang M, Zhang H (2000b) A comparison between photospheric and chromospheric quiet-Sun magnetograms. Sol Phys 194:19-28

Zhang H, Yang S (2013) Distribution of magnetic helicity flux with solar cycles. Astrophys J 763:105. doi:10.1088/0004-637X/763/2/105

Zhang M, Zhang HQ, Ai GX, Wang HN (1999) Different spatial structures between network regions and active regions indicated by TRACE $171 \AA$ observation. Sol Phys 190:79-90. doi:10.1023/A: 1005276217089

Zhang Y, Liu J, Zhang H (2008) Relationship between rotating sunspots and flares. Sol Phys 247:39-52. doi:10.1007/s11207-007-9089-0

Zhang H, Sakurai T, Pevtsov A, Gao Y, Xu H, Sokoloff DD, Kuzanyan K (2010a) A new dynamo pattern revealed by solar helical magnetic fields. Mon Not R Astron Soc 402:L30-L33. doi:10.1111/j. 1745-3933.2009.00793.x

Zhang H, Yang S, Gao Y, Su J, Sokoloff DD, Kuzanyan K (2010b) Large-scale soft X-ray loops and their magnetic chirality in both hemispheres. Astrophys J 719:1955-1963. doi:10.1088/0004-637X/719/2/ 1955

Zhang L, Mursula K, Usoskin I, Wang H (2011) Global analysis of active longitudes of sunspots. Astron Astrophys 529:A23. doi:10.1051/0004-6361/201015255

Zhao M, Wang J-X, Jin C-L, Zhou G-P (2009) Magnetic non-potentiality on the quiet Sun and the filigree. Res Astron Astrophys 9:933-944. doi:10.1088/1674-4527/9/8/009

Zhao X, Hoeksema JT (1996) Effect of coronal mass ejections on the structure of the heliospheric current sheet. J Geophys Res 101:4825-4834. doi:10.1029/95JA03568

Zheng R, Jiang YC, Yang LH, Bi Y (2011) The coronal mass ejection associated with the loop eruption and coronal dimmings on 2009 December 13. Astrophys Space Sci 332:81-89. doi:10.1007/ s10509-010-0505-9 
Zhou GP, Wang JX, Jin CL (2010) Solar intranetwork magnetic elements: evolution and lifetime. Sol Phys 267:63-73. doi:10.1007/s11207-010-9641-1

Zhukov AN, Saez F, Lamy P, Llebaria A, Stenborg G (2008) The origin of polar streamers in the solar corona. Astrophys J 680:1532-1541. doi:10.1086/587924

Zirin H (1987) Weak solar fields and their connection to the solar cycle. Sol Phys 110:101-107. doi:10. 1007/BF00148205

Zwaan C (1987) Elements and patterns in the solar magnetic field. Ann Rev Astron Astrophys 25:83-111. doi:10.1146/annurev.aa.25.090187.000503 